\title{
Miriam Günderoth
}

\section{Kindeswohlgefährdung}

Die Umsetzung des Schutzauftrages in der verbandlichen Jugendarbeit

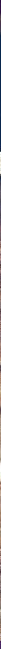




\section{Miriam Günderoth}

Kindeswohlgefährdung 
$\mathrm{D}$ ie Reihe »Angewandte Sexualwissenschaft « sucht den Dialog: Sie ist interdisziplinär angelegt und zielt insbesondere auf die Verbindung von Theorie und Praxis. Vertreter_innen aus wissenschaftlichen Institutionen und aus Praxisprojekten wie Beratungsstellen und Selbstorganisationen kommen auf Augenhöhe miteinander ins Gespräch. Auf diese Weise sollen die bisher oft langwierigen Transferprozesse verringert werden, durch die praktische Erfahrungen erst spät in wissenschaftlichen Institutionen Eingang finden. Gleichzeitig kann die Wissenschaft so zur Fundierung und Kontextualisierung neuer Konzepte beitragen.

Der Reihe liegt ein positives Verständnis von Sexualität zugrunde. Der Fokus liegt auf der Frage, wie ein selbstbestimmter und wertschätzender Umgang mit Geschlecht und Sexualität in der Gesellschaft gefördert werden kann. Sexualität wird dabei in ihrer Eingebundenheit in gesellschaftliche Zusammenhänge betrachtet: In der modernen bürgerlichen Gesellschaft ist sie ein Lebensbereich, in dem sich Geschlechter-, Klassen- und rassistische Verhältnisse sowie weltanschauliche Vorgaben - oft konflikthaft - verschränken. Zugleich erfolgen hier Aushandlungen über die offene und Vielfalt akzeptierende Fortentwicklung der Gesellschaft.

\author{
BAND 9 \\ Angewandte Sexualwissenschaft \\ Herausgegeben von Ulrike Busch, Harald Stumpe, \\ Heinz-Jürgen Voß und Konrad Weller, \\ Institut für Angewandte Sexualwissenschaft \\ an der Hochschule Merseburg
}




\author{
Miriam Günderoth
}

\title{
Kindeswohlgefährdung
}

Die Umsetzung des Schutzauftrages in der verbandlichen Jugendarbeit

Psychosozial-Verlag 
Die Open-Access-Publikation wurde durch eine Förderung des

Bundesministerium für Bildung und Forschung ermöglicht.

Dieses Werk ist lizenziert unter der Creative Commons Attribution-Non-

Commercial-NoDerivs 3.0 DE Lizenz (CC BY-NC-ND 3.0 DE).

Diese Lizenz erlaubt die private Nutzung und unveränderte Weitergabe, verbietet jedoch die Bearbeitung und kommerzielle Nutzung.

Weitere Informationen finden Sie unter: https://creativecommons.org/ licenses/by-nc-nd/3.0/de/

$$
\text { (c) } \underset{\mathrm{BY}}{\mathrm{BY}}\left(\mathrm{ND}_{\mathrm{ND}}\right.
$$

Bibliografische Information der Deutschen Nationalbibliothek Die Deutsche Nationalbibliothek verzeichnet diese Publikation in der Deutschen Nationalbibliografie; detaillierte bibliografische

Daten sind im Internet über http://dnb.d-nb.de abrufbar.

\section{Originalausgabe}

C 2017 Psychosozial-Verlag, Gießen

E-Mail: info@psychosozial-verlag.de www.psychosozial-verlag.de

Alle Rechte vorbehalten. Kein Teil des Werkes darf in irgendeiner Form (durch Fotografie, Mikrofilm oder andere Verfahren) ohne schriftliche Genehmigung des Verlages reproduziert oder unter Verwendung elektronischer Systeme verarbeitet, vervielfältigt oder verbreitet werden.

Umschlagabbildung: »Kinderfüße« (C) monropic/fotolia.com Innenlayout und Umschlaggestaltung nach Entwürfen von

Hanspeter Ludwig, Wetzlar www.imaginary-world.de Satz: metiTEC-Software, me-ti GmbH, Berlin ISBN 978-3-8379-2639-2 (Print) ISBN 978-3-8379-7279-5 (E-Book-PDF) ISSN 2367-2420 (Print) https://doi.org/10.30820/9783837972795 


\section{Inhalt}

Vorwort

\section{Rechtliche Grundlagen}

1. Summarisch: Der Weg zum Kinder- und Jugendhilfegesetz (SGB VIII)

2. Das SGB VIII - Kinder- und Jugendhilfe

3. Das Bundeskinderschutzgesetz und seine Auswirkung auf das SGB VIII, fokussiert auf die Jugendverbandsarbeit

4. Der §8a SGB VIII - Schutzauftrag bei Kindeswohlgefährdung

5. Der §72a SGB VIII - Tätigkeitsausschluss einschlägig vorbestrafter Personen

\section{Bedeutung zentraler Begriffe}

1. Sexualisierte Gewalt 33

2. Fachkraft und Eignung nach $\S 72$ SGB VIII 36

3. Ehrenamtlich Tätige in der verbandlichen Jugendarbeit 38

4. Kindeswohl 38 
5. Kindeswohlgefährdung 39

6. Formen der Kindeswohlgefährdung und mögliche Signale $\quad 40$

7. Gewichtige Anhaltspunkte 49

8. Risikoeinschätzung 50

9. Risikofaktoren für die Gefährdung des Kindeswohls 51

10. Datenschutz 54

\section{Die Bedeutung des Schutzauftrags} für die verbandliche Jugendarbeit

1. Vereinbarungen mit dem Jugendamt 64

2. Regelungen und Transparenz im Verband 66

3. Vorgehen bei Verdachtsfällen 68

4. Beauftragung von ehrenamtlich Mitarbeitenden 75

5. Ausbildung von ehrenamtlich Mitarbeitenden 79

\section{Konzeptionelle Überlegungen zur Einbindung} des Schutzauftrages in die Ausbildung von ehrenamtlich Mitarbeitenden

1. Bausteine für den Bereich Entwicklungspsychologie 87

2. Baustein rechtliche Grundlagen 90

3. Baustein Strukturen der verbandlichen Jugendarbeit 91

4. Baustein Vertiefung 92

5. Überlegungen für Mitarbeiter_innenabende/-kreise 94

6. Schulungsmodul für die Freizeitvorbereitung 95

$\begin{array}{ll}\text { Fazit } & 99\end{array}$

$\begin{array}{ll}\text { Literatur } & 101\end{array}$

$\begin{array}{ll}\text { Anhang } & 107\end{array}$ 


\section{Vorwort}

Das Buch entstand auf Basis langjähriger Erfahrungen in der verbandlichen Jugendarbeit und verbunden mit einem Studium der Sexualpädagogik und Familienplanung (heute: Angewandte Sexualwissenschaft) an der Hochschule Merseburg, das ich 2011 abschloss. Zu meinen Aufgaben als Jugendreferentin gehörten neben der Verantwortung für die pädagogische Gruppen- und Freizeitarbeit auch die Begleitung und Ausbildung von ehrenamtlich Mitarbeitenden. Unter dem Eindruck der Aufdeckungen langjährigen sexuellen Missbrauchs an einigen Internaten, der mit den Ereignissen verbundenen Verunsicherungen von Fachkräften in der Jugendverbandsarbeit und der Konfrontation mit Fragen des Umgangs mit dem Kinderschutz im eigenen Verband, sah und sehe ich den Bedarf der Veröffentlichung dieser aktuellen praxisund bedarfsorientierten Handreichung. Sie enthält viele Hinweise und richtet sich insbesondere an Fachkräfte und ehrenamtlich Tätige im Bereich der verbandlichen Jugendarbeit.

2010 gab es viele Überlegungen zu dem Thema. Wenige beschäftigten sich allerdings mit der verbandlichen Jugendarbeit. Das änderte sich in den darauffolgenden Jahren, in denen einige Bücher und Handreichungen entstanden.

Gesetzliche Änderungen, unter anderem die Einführung des Bundeskinderschutzgesetzes, sind bei der Überarbeitung aufgenommen worden. Auch findet der \$72a SGB VIII ausführliche Beachtung auch über die Frage von Führungszeugnissen für ehrenamtlich Mitarbeitende in der Kinder- und Jugendhilfe hinausgehend. Heute spielt der 
Bereich der Führungszeugnisse oftmals eine größere Rolle in der verbandlichen Jugendarbeit als die Handlungskonzepte bei Verdacht auf Kindeswohlgefährdung. Das mag daran liegen, dass Institutionen der verbandlichen Jugendarbeit in den meisten Fällen keine Träger von Einrichtungen und Diensten sind und damit keine Vereinbarungen nach §8a SGB VIII mit dem öffentlichen Jugendhilfeträger abschließen.

Kindesmisshandlung, Gewalt gegen Kinder, sexuelle Grenzverletzungen und Missbrauch umspannen ein weites Themenfeld mit vielen angrenzenden Bereichen, die in der vorliegenden Publikation angepasst gewichtet sind, gerade unter der Prämisse, gut für die Schulungsarbeit im Verband geeignet zu sein. Ein großer Teil der Arbeit beschäftigt sich mit der Auseinandersetzung mit dem Gesetzestext des $\$ 8$ a SGB VIII und den zugehörigen Kommentaren. Historisch gesehen ist der Kinderschutz noch nicht sehr alt, darum umso interessanter ist dessen Entwicklung bis zum heutigen Tag. Die Auseinandersetzung hiermit ist eine Grundlage für die Vermittlung der Inhalte innerhalb der Schulungsarbeit.

Keine Beachtung findet hingegen der Bereich der $\gg$ Frühen Hilfen «. Hierzu gibt es viel Literatur, was daran liegt, dass im Kleinkindalter Vernachlässigung und Gefährdung des Kindeswohls gravierendere Folgen - bis hin zum Tod - haben kann; das gilt nicht unbedingt für das Jugendalter. Die Zielgruppen verbandlicher Jugendarbeit sind in der Regel Kinder und Jugendliche ab dem sechsten Lebensjahr, in wenigen Fällen sind sie jünger. Freizeiten mit externer Übernachtung werden häufig ab dem achten Lebensjahr angeboten.

Nicht abgeschlossen ist die Auseinandersetzung mit der Thematik der Kindeswohlgefährdung und sexualisierten Gewalt im Jugendalter. In der verbandlichen Jugendarbeit sind Jugendliche Teilnehmende und Mitarbeitende und können Betroffene von Grenzüberschreitungen und sexualisierter Gewalt innerhalb des Verbandes sein, aber selber auch Täter_innen werden. Am Ende der Arbeit werden einige Impulse und Gedanken, die diesen Bereich betreffen, aufgegriffen.

Schutzmaßnahmen innerhalb eines Jugendverbandes müssen auf Grundlage einer reflektierten Analyse der Organisations- und Angebotsstruktur, einer sogenannten Risikoanalyse, individuell entwickelt werden. Dafür ist ein längerer Prozess erforderlich; die dafür notwendigen Überlegungen finden sich an anderer Stelle - insbesondere möchte 
ich auf den Beitrag »Prävention von sexueller Gewalt im Ehrenamtssektor « von Beate Steinbach im Sammelband Kompendium »Sexueller Missbrauch in Institutionen «. Entstehungsbedingungen, Prävention und Intervention verweisen (Fegert \& Wolff, 2015, S. 186-196). 


\section{Rechtliche Grundlagen}

Für die Auseinandersetzung mit dem Schutzauftrag bei Kindeswohlgefährdung und dem Schutz vor Grenzüberschreitungen und sexualisierter Gewalt sind neben den im achten Buch des Sozialgesetzbuchs (SGB VIII) verankerten Paragrafen 8a und 72a verschiedene relevante Gesetzestexte zu beachten: Zuerst ist der in Artikel 19 der UN-Kinderrechtskonvention beschriebene Schutz vor Gewaltanwendung, Misshandlung und Verwahrlosung zu erwähnen. Aber auch das im Grundgesetz verankerte Elternrecht auf Erziehung und Pflege des Kindes nach Art. 6 Abs. 2 ist bedeutsam. Darüber obliegt dem Staat das sogenannte »Wächteramt « nach $\$ 1$ Abs. 3 Nr. 3 SGB VIII. Für das Kinderschutzsystem in Deutschland ist zudem der $\$ 1666$ BGB wichtig, der die Handlungsmöglichkeiten des Familiengerichts definiert, Maßnahmen zum Schutz von Kindern und Jugendlichen anzuordnen, sofern die Eltern nicht gewillt oder fähig sind, das Wohl des Kindes zu gewährleisten (vgl. Kindler, 2007, S. 5). Schließlich ist der $\$ 1631$ Abs. 2 des BGB relevant. Seine Geschichte ist im Zusammenhang mit dem Kindeswohl erhellend. So wandelte er sich von 1900 bis 2000 (vgl. Tabelle 1) von einem ausdrücklichen Recht des Vaters, » angemessene Zuchtmittel gegen das Kind « anzuwenden, zu dem Recht des Kindes » auf gewaltfreie Erziehung « (vgl. Maywald, 2014, S. 7f.).

1958 Im Zuge des Gleichberechtigungsgesetzes wurde das ausdrückliche Recht des Vaters, seine Kinder zu erzieherischen Zwecken zu schlagen, ersatzlos gestrichen, weil es nicht gleichermaßen der Mutter zuerkannt war! 
1980 Die Passage »Entwürdigende Erziehungsmaßnahmen sind unzulässig « wurde eingefügt. Damit waren körperliche Bestrafungen gemeint, die das Selbstwertgefühl des Kindes massiv verletzen und eine Entwürdigung und Demütigung darstellen.

1998 »Entwürdigende Erziehungsmaßnahmen, insbesondere körperliche und seelische Misshandlungen, sind unzulässig.« Mit der Erweiterung und Zuspitzung wurde den Erkenntnissen Rechnung getragen, dass weiterhin Kindern elterliche Gewalt angetan wurde, die gerechtfertigt wurde, indem eine Unterscheidung zwischen entwürdigenden und nichtentwürdigenden Erziehungsmaßnahmen getroffen wurde.

2000 »Kinder haben ein Recht auf gewaltfreie Erziehung. Körperliche Bestrafung, seelische Verletzung und andere entwürdigende Maßnahmen sind unzulässig. « Mit der Verabschiedung des » Gesetzes zur Ächtung der Gewalt in der Erziehung und zur Änderung des Kindesunterhaltsrechts « wurde im November 2000 auch der Abs. 2 des $\$ 1631$ BGB neu gefasst. Mit dem $\$ 16$ Abs. 1 Satz 3 SGB VIII wurde parallel auch die Förderung der Erziehung in der Familie um die Aufgabe erweitert, Familien in Konfliktsituationen einen gewaltfreien Weg aufzuzeigen mit dem Ziel, Eltern zu begleiten und nicht zu ächten.

Tabelle 1: Übersicht über zentrale gesetzliche Veränderungen des §1631 Abs. 2 $B G B$

Rechtliche Unterscheidungen zwischen Kindern und Erwachsenen gibt es auch heute noch - Kinder werden von Erwachsenen weniger ernst genommen -, dennoch haben Kinder in ihrer rechtlichen Stellung, besonders durch die Beachtung der UN-Kinderrechtskonvention, eine Aufwertung erfahren. Die Tatsache, »Kinder als Subjekte und Träger eigener Rechte anzusehen und Gewalt in der Erziehung nicht zuzulassen, ist historisch neu und auch heute im Bewusstsein vieler Erwachsener nicht fest verankert « (Maywald, 2014, S. 5). Das wird etwa 
dadurch deutlich, dass in der Bundesrepublik Deutschland erst 1990 entsprechende rechtliche Regelungen eingeführt wurden - zentral ist hier das Inkrafttreten des Kinder- und Jugendhilfegesetzes (SGB VIII). Im SGB VIII werden Kinder und Jugendliche ausdrücklich als Träger eigener Rechte benannt. Heute sichern internationale und bundesweite Rechte Kindern und Jugendlichen das Recht auf Schutz vor Gefahren zu. Auch, dass das Elternrecht der alleinigen Erziehung und Pflege ausdrücklich dem Schutz und zum Wohle des Kindes dienen soll, stand bis vor wenigen Jahren nicht explizit im Gesetzestext.

Der Schutz der Kinder vor Gefahren und Missbrauch ist eng mit der Stellung des Kindes in der zeitgenössischen Pädagogik verknüpft. So wurde die Kindheit als ein eigenständiger Lebensabschnitt erst im Zeitalter der Aufklärung »entdeckt «. Die weitergehende historische Dimension der Entwicklung der Kindheit soll aber nicht Inhalt des Buches sein. Im Folgenden wird nur summarisch auf die Historie der jüngeren Vergangenheit eingegangen. Schwerpunkt wird die historische Entwicklung der UN-Kinderrechtskonvention sein. Anschließend werden insbesondere die bezüglich des Kinderschutzes bedeutsamen Inhalte des SGB VIII fokussiert. Die $\$ \$ 8$ a und 72a SGB VIII finden dabei besondere Beachtung.

\section{Summarisch: Der Weg zum Kinder- und Jugendhilfegesetz (SGB VIII)}

Was wir heute unter Kindesmisshandlung verstehen, gehörte bis ins 20. Jahrhundert zum Teil zu den gängigen pädagogischen Umgangsweisen mit Kindern und Jugendlichen. Die Maßnahmen zielten auf die gewaltsame Unterwerfung und Disziplinierung der Kinder ab. Frank Meier beschreibt jedoch, dass auch schon im Mittelalter - ich beziehe mich hier ausschließlich auf das lateinische Mittelalter, im arabischen Mittelalter gab es darüber hinausgehende Betrachtungen unterschiedliche Ansichten über Erziehungsmethoden und -mittel vorhanden waren. So sprach Jean Carlier Gerson (1363-1429) von einer Erziehung »vom Wesen des Kindes her und trat für einen partnerschaftlichen Umgang ein « (Meier, 2006, S. 136). Verschiedene Dichter und Denker (z. B. F. Schiller, Chr. M. Wieland, J. W. v. Goethe) be- 
schrieben den Zusammenhang von körperlicher Züchtigung und der seelischen Entwicklung des Menschen. Die negativen Folgen der Gewaltausübung für den Charakter des Menschen waren schon vor dem 20. Jahrhundert bekannt (vgl. ebd., S. 137). Es gab aber auch andere Stimmen, welche die »harte Hand « bei der Erziehung als notwendig ansahen und die Rute als Mittel der Wahl bei Ungehorsam empfahlen. Um Kindern gute Sitten zu vermitteln, waren Einschüchterung und Drohung ebenso an der Tagesordnung wie Züchtigungen. So waren Bilder und in Sandstein gehauene Szenen an Kircheneingängen tägliche Mahnung zur Gehorsamkeit gegenüber den Eltern, besonders gegenüber dem Vater, unter dessen Gewalt die Kinder standen (vgl. ebd., S. 138ff.).

Das 19. Jahrhundert gilt als Beginn der neuen Pädagogik. Politisch war die Zeit von Revolutionen in Europa geprägt. Auf gesellschaftspolitischer Ebene bereiteten Reformen den modernen Verwaltungsstaat vor. Familienformen wurden vielfältiger, Familie veränderte und verbürgerlichte sich, wobei weiterhin patriarchale Strukturen herrschten und dem Gesetz des Vaters Folge zu leisten war. Die Kinder unterstanden der » väterlichen Gewalt, die zugleich ein Macht-, Verantwortungsund Abhängigkeitsverhältnis begründete « (Hoyer, 2015, S. 53). Neben den bürgerlichen Familien und den Arbeiterfamilien in den Städten gab es auf dem Land die großbäuerlichen Familien, bei welchen die Mehrgenerationenhaushalte mit strengen patriarchalen Strukturen vorherrschte, in dem auch die strenge Erziehung mit körperlichen Züchtigungen ihren Platz hatte. Die Bauernbefreiung ermöglichte eine neue Lebensform: die der Landarbeiterfamilien. Sie lebten in ökonomischer Abhängigkeit zu den Landwirtschaftsbetrieben und waren ähnlich wie die bürgerliche Familie von der Trennung von Haus und Arbeit betroffen. Familie war hier, entgegen den großbäuerlichen Familien, auf die Kernfamilie begrenzt (vgl. ebd., S. 84f.). Die Veränderungen in den Familien wirkten sich in der Folgezeit unterschiedlich auf die Kinder und deren Rechte und Pflichten in Bezug auf den familiären Lebensunterhalt aus. »Je härter die Versorgungsnot wurde, desto mehr Buben und Mädchen mussten bei der Feldarbeit, in der Hauswirtschaft und den Alltagsverrichtungen anpacken « (ebd., S. 107).

Ein Wendepunkt in der Erziehung und im Verständnis auf dem Weg zu einer gewaltfreien Erziehung war am Ende des 19. Jahrhunderts. Die 
bis dahin gängige wissenschaftsorientierte Vermittlung der Lerninhalte in den Schulen wurde in unterschiedlicher Weise reformiert und es entstanden nach 1900 vielfältige neue methodische Ansätze, die eine handlungsorientierte und ganzheitliche Vermittlung von Wissen verfolgten. In den Folgejahren entstand daraus die reformpädagogische Bewegung. Ihre Fürsprecher forderten einheitlich, wenn auch mit unterschiedlichen Denkansetzen und Zielen, »eine kinderfreundlichere Schule, die der Selbsttätigkeit und dem spezifischen Lernverhalten von Heranwachsenden Rechnung trage «(ebd., S. 131). 1900 erklärte die schwedische Pädagogin Ellen Key (1849-1926) das 20. Jahrhundert mit ihrem gleichnamigen Buch zum Jahrhundert des Kindes. Durch die deutsche Übersetzung im Jahr 1902 fanden ihre Ansichten große Beachtung. Sie schreibt über die Verantwortung zu Beginn des neuen Jahrhunderts, von der Aufgabe, Erziehung von Kindern und Jugendlichen als »höchste Angelegenheit des Volkes « (Key, 1902, S. 8) anzusehen, da sie die Zukunft sind. Gleichzeitig kritisiert sie, dass »in Wirklichkeit [...] sowohl in der Familie wie in den Schulen und im Staate ganz andere Werte in den Vordergrund gestellt « (ebd.) werden. Neben aller Kritik zu ihrer ausgrenzenden und eugenischen Haltung gegenüber Kindern, die physisch unheilbare Krankheiten und Missbildungen hatten, war sie dennoch durch ihre Forderung auf Gewaltverzicht in der Erziehung eine Vorreiterin zur heutigen Erziehungshaltung. Die Aussage, dass das 20. Jahrhundert das Jahrhundert des Kindes sei, würde heute nicht mehr getroffen werden, denn es gab nach wie vor Ausbeutung, Prostitution und gewaltvolle Erziehung von Kindern und auch heute noch sterben täglich Kinder an Unterernährung und vermeidbaren Erkrankungen. In der Geschichte der Kinderrechte ist das 20. Jahrhundert dennoch die wichtigste Epoche (vgl. Unicef, 2015, S. 1). Einige wichtige Ereignisse:

Während der Weimarer Republik (1918-1933) kam der Gedanke auf, dass nicht die soziale Herkunft, sondern die Neigungen und Fähigkeiten des einzelnen Kindes über den Grad der Bildung entscheiden sollten. Zur gleichen Zeit wurden die ersten Diskussionen über Kinderrechte im Völkerbund geführt. In ganz Europa beschäftigten sich Menschen mit den Rechten für Kinder. So forderte Janusz Korczak im Jahr 1919 Grundrechte für Kinder. Sigrid Tschörpe-Scheffler fasst die Forderungen Korczaks zusammen: Es gehe um das Recht des Kindes 
» als einmaliger Mensch, der keinem Entwurf entsprechen muss, der Geheimnisse und Träume haben darf, wahrgenommen zu werden [...] [und um das] Recht auf Zeit, auf Raum, auf lebendige Umgangserfahrungen, auf Trauer und Schmerz, das Mitspracherecht in allen das Kind betreffenden Lebensbereichen « (Tschörpe-Scheffler, 2009, S. 31).

Eglantyne Jebb, die Begründerin von »Save the Children Fund « in England, entwickelte eine Satzung für Kinder und reichte diese zur Abstimmung in den Völkerbund ein. Am 26. September 1924 wurde diese Charta als Genfer Erklärung verabschiedet. Sie enthielt grundlegende Rechte des Kindes, hatte jedoch keine rechtliche Verbindlichkeit (vgl. Unicef, 2015, S. 1):

I. Das Kind soll in der Lage sein, sich sowohl in materieller wie in geistiger Hinsicht in natürlicher Weise zu entwickeln.

II. Das hungernde Kind soll genährt werden; das kranke Kind soll gepflegt werden; das zurückgebliebene Kind soll ermuntert werden; das verirrte Kind soll auf den guten Weg geführt werden; das verwaiste und verlassene Kind soll aufgenommen und unterstützt werden.

III. Dem Kind soll in Zeiten der Not zuerst Hilfe zuteil werden.

IV. Das Kind soll in die Lage versetzt werden, seinen Lebensunterhalt zu verdienen und soll gegen jede Ausbeutung geschützt werden.

V. Das Kind soll in dem Gedanken erzogen werden, seine besten Kräfte in den Dienst seiner Mitmenschen zu stellen. ${ }^{1}$

Der zweite Weltkrieg setzte die bis dahin erwirkten Vereinbarungen zu den Kinderrechten außer Kraft. Der Völkerbund löste sich 1946 auf und an seine Stelle trat die Generalversammlung der Vereinten Nationen.

Die Genfer Erklärung von 1924 wurde in der Generalversammlung der Vereinten Nationen im Jahr 1948 wieder aufgegriffen und diente als Vorbild für die Erklärung über die Rechte des Kindes. Diese Erklärung wurde am 20. November 1959 als Resolution der Generalversammlung der Vereinten Nationen beschlossen. Mit zehn Grundsätzen wurden die Rechte des Kindes auf besonderen Schutz bekräftigt und die Anerkennung von Kindern als Rechtssubjekte mit eigenen Schutz- 
und Anspruchsrechten festgeschrieben. In diesen Grundsätzen finden sich das Recht auf gesunde und natürliche Entwicklung in Freiheit und Würde; die Rechte auf ausreichende Ernährung, Wohnung, Erholung und ärztliche Betreuung; auf ein Aufwachsen in einer Atmosphäre der Zuneigung und Sicherheit in der Obhut und Verantwortung seiner Eltern; den Anspruch auf Erziehung und den Schutz vor jeder Art von Vernachlässigung, Grausamkeit und Ausnutzung (vgl. Erklärung der Rechte des Kindes, 1959).

1979 wurde als Internationales Jahr des Kindes bestimmt. »Auf Grundlage einer polnischen Initiative [...] wurde eine Arbeitsgruppe der Menschenrechtskommission bei den Vereinten Nationen damit beauftragt, eine Konvention über die Rechte des Kindes zu erarbeiten « (Maywald, 2014, S. 6). In 54 Artikeln wurden das völkerrechtlich verbindliche Übereinkommen über die Rechte des Kindes (folgend: UNKinderrechtskonvention) beschrieben und am 20. November 1989 in der 44. Vollversammlung der Vereinten Nationen einstimmig verabschiedet. Unter dem Begriff »Kind « verstehen die Vereinten Nationen in der UN-Kinderrechtskonvention jeden Menschen, » der das achtzehnte Lebensjahr noch nicht vollendet hat, soweit die Volljährigkeit nach dem auf das Kind anzuwendenden Recht nicht früher eintritt « (UNKinderrechtskonvention, Art. 1). Heute ist die UN-Kinderrechtskonvention die höchst ratifizierte UN-Konvention. Bis 2016 haben alle UN-Staaten - bis auf die USA - die von ihren Parlamenten notwendige Zustimmung zum Beitritt der Kinderrechtskonvention der Vereinten Nationen gegeben und diesen unterschrieben. Dieser Vertrag ist wie auch die internationale Menschenrechtscharta von 1959 völkerrechtlich bindend, jedoch ist es schwierig, Verstöße dagegen zu ahnden, da es keine internationale Gerichtsbarkeit gibt (vgl. Fegert et al., 2015, S. 31). Deutschland gehörte zu den ersten Nationen, welche die UNKinderrechtskonvention am 26. Januar 1990 unterzeichneten. Die notwendige Zustimmung von Bundestag und Bundesrat erstreckte sich durch die kontrovers geführten pädagogischen und politischen Diskussionen über zwei Jahre. Inhalt der Diskussionen war die Uneinigkeit über die Durchführbarkeit des Partizipationsgedankens und darüber, ob Kinder grundsätzlich im Besitz der kognitiven und moralischen Voraussetzungen sind, um sich an Entscheidungsprozessen beteiligen zu können (vgl. Tshörpe-Scheffler, 2009, S. 32ff.). Am 5. April 1992 trat 
die UN-Kinderrechtskonvention für Deutschland in Kraft. Und damit ist sie bei Entscheidungen von Behörden und Gerichten zu beachten (vgl. Fegert et al., 2015, S. 167).

Die Inhalte der Artikel 3 und 12 beschreiben dieses Recht:

\section{》Artikel 3 - Wobl des Kindes}

Bei allen Maßnahmen, die Kinder betreffen, [...] ist das Wohl des Kindes ein Gesichtspunkt, der vorrangig zu berücksichtigen.

Die Vertragsstaaten verpflichten sich, dem Kind unter Berücksichtigung der Rechte und Pflichten seiner Eltern, seines Vormunds oder anderer für das Kind gesetzlich verantwortlicher Personen den Schutz und die Fürsorge zu gewährleisten, die zu seinem Wohlergehen notwendig sind; zu diesem Zweck treffen sie alle geeigneten Gesetzgebungsund Verwaltungsmaßnahmen.«

\section{$\gg$ Artikel 12 - Berücksichtigung des Kindeswillens}

Die Vertragsstaaten sichern dem Kind, das fähig ist, sich eine eigene Meinung zu bilden, das Recht zu, diese Meinung in allen das Kind berührenden Angelegenheiten frei zu äußern, und berücksichtigen die Meinung des Kindes angemessen und entsprechend seinem Alter und seiner Reife.

$\mathrm{Zu}$ diesem Zweck wird dem Kind insbesondere Gelegenheit gegeben, in allen das Kind berührenden Gerichts- oder Verwaltungsverfahren entweder unmittelbar oder durch einen Vertreter oder eine geeignete Stelle im Einklang mit den innerstaatlichen Verfahrensvorschriften gehört zu werden.«

Insbesondere die vorrangige Berücksichtigung des Wohl des Kindes bei allen Maßnahmen, die Kinder betreffen, ist in letzter Konsequenz im deutschen Rechtsystem nicht berücksichtigt, auch wenn im $\$ 8$ Abs. 1 SGB VIII aufgenommen ist, dass

»Kinder und Jugendliche [...] entsprechend ihrem Entwicklungsstand an allen sie betreffenden Entscheidungen der öffentlichen Jugendhilfe zu beteiligen [sind]. Sie sind in geeigneter Weise auf ihre Rechte im Verwaltungsverfahren sowie im Verfahren vor dem Familiengericht und dem Verwaltungsgericht hinzuweisen.« 
Reinald Eichholz sieht hier besonders auch die Verwaltungspraxis als problematisch an, bei deren Entscheidungen viele Interessenslagen berücksichtigt werden, »nicht jedoch den Vorrang des Kindeswohls bei der Verwirklichung seines Rechts auf chancengleiche Bildung > ohne jede Diskriminierung « (Eichholz, 2009, S. 14).

Mit einer Vorbehaltserklärung der Bundesregierung beschnitt Deutschland die Gültigkeit der in der UN-Kinderrechtskonvention festgelegten Rechte. Damit gestand es insbesondere Kindern ohne deutschen Pass nicht die gleichen Rechte zu wie deutschen Kindern.

Das betrifft neben dem schon erwähnten Artikel 3 auch den Artikel 22, der sich mit der Thematik der Flüchtlingskinder beschäftigt. Darin heißt es:

»Die Vertragsstaaten treffen geeignete Maßnahmen, um sicherzustellen, dass ein Kind, das die Rechtsstellung eines Flüchtlings begehrt oder nach Maßgabe der anzuwendenden Regeln und Verfahren des Völkerrechts oder des innerstaatlichen Rechts als Flüchtling angesehen wird; angemessenen Schutz und humanitäre Hilfe bei der Wahrnehmung der Rechte erhält, die in diesem Übereinkommen oder in anderen internationalen Übereinkünften über Menschenrechte oder über humanitäre Fragen, denen die genannten Staaten als Vertragsparteien angehören, festgelegt sind, und zwar unabhängig davon, ob es sich in Begleitung seiner Eltern oder einer anderen Person befindet oder nicht. «

Dieser Vorbehalt wurde erst im Mai 2010 zurückgenommen. Allerdings dauerte es noch bis November 2015, bis auch die gesetzlichen Regelungen zur Verbesserung der Situation von jungen unbegleiteten Flüchtlingen in Kraft getreten sind.

In Deutschland entstand neben der von der UN geführten Debatte eine eigenständige Diskussion über Kinderrechte, die zur Einführung des SGB VIII führte. Es trat zum 1. Januar 1991 in Kraft und löste das bis dahin geltende Jugendwohlfahrtsgesetz ab. ${ }^{2}$ Im Gegensatz zu seinen Vorläufern ist das SGB VIII ein präventiv orientiertes Leistungsrecht. Die Förderangebote und präventiven Leistungen wurden

2 Im Rahmen des Einigungsvertrags trat es auf dem Gebiet der ehemaligen DDR schon am 3. Oktober 1990 in Kraft. 
ausgebaut, während repressive Eingriffe in die Belange der Eltern und Kinder eine deutlich geringere Rolle spielen als es beim davor geltenden Jugendwohlfahrtsgesetz (JWG) und dessen Vorgänger, dem Reichsjugendwohlfahrtsgesetz (RJWG), der Fall war. Diese waren vorwiegend ordnungsrechtliche Eingriffsinstrumentarien mit nur wenigen Hilfen, dafür mit mehr staatlicher Kontrolle. Leistungsansprüche auf Hilfeleistungen waren eher systemfremd und spielten nur am Rande eine Rolle. Mit der Umorientierung und dem neuen Grundverständnis des Kinderund Jugendhilferechts vollzog der Gesetzgeber einen Paradigmenwechsel in der Jugendhilfe. Das SGB VIII kann also als ein Instrument zur Vorbeugung, Hilfestellung und Unterstützung von Personensorgeberechtigten und Erziehungsberechtigten und zum Schutz von Kindern und Jugendlichen gesehen werden (vgl. Kunkel, 2015, Rn. 40, S. 37).

In der Öffentlichkeit verstärkt wahrgenommene Fälle von Kindesvernachlässigung in den darauffolgenden Jahren führten dazu, dass sich eine Gesetzesinitiative bildete, um den Kinder- und Jugendschutz zu verbessern. Daraus entstand 2005 das Kinder- und Jugendhilfeweiterentwicklungsgesetz (KICK), das vor allem mit seinen neu eingefügten $\$ \$ 8$ a und $72 \mathrm{a}$ im SGB VIII auf diese Entwicklung reagierte.

Mit dem KICK wurde das SGB VIII umgestaltet bzw. neu geordnet und neue Regelungen hinzugefügt. Dem Gesetzgeber war es ein zentrales Anliegen, den Schutz von Kindern und Jugendlichen weiter zu optimieren. Die Einführung der $\$ \$ 8$ a (Schutzauftrag), 36a (Steuerungsverantwortung des Jugendamtes bezüglich der Hilfen zur Erziehung), 43 (Erlaubnis zur Kindertagespflege) und 72a (persönliche Eignung von Fachkräften) in das SGB VIII sollten zu dieser Zielverfolgung beitragen. Die schon vorhandenen Hilfeleistungen sollten dadurch optimiert werden und dabei helfen, dass Gefährdungen früher erkannt werden. Auch der Datenschutz wurde im Zusammenhang mit dem Kinderschutz aufgegriffen und geregelt. Durch die $\$ \$ 62$ und 65 SGB VIII wurde die Erhebung und Weitergabe von Sozialdaten bei Kindeswohlgefährdung erleichtert (vgl. ebd., Rn. 35, S. 32).

Gleichzeitig mit der Einführung der Verbesserungen vollzog der Gesetzgeber einen weiteren Paradigmenwechsel: War bisher der öffentliche Träger (i. d. R. das Jugendamt) für den Kinder- und Jugendschutz verantwortlich, wurde nun die Verantwortung auch auf die freien Träger der Kinder- und Jugendhilfe ausgeweitet. Mit der neuen Gesetzgebung 
sind die freien Träger verpflichtet, gewichtigen Anzeichen nachzugehen und entsprechende Maßnahmen zum Schutz vor Gefährdung des Wohls von Kindern und Jugendlichen einzuleiten.

Obwohl in der fachlichen Debatte schon in den 1970ern und 1980ern das Phänomen des sexuellen Missbrauchs von Frauen und Mädchen in ihren Familien und dem sozialen Nahraum thematisiert wurde, gab es keine größere öffentliche Debatte. Auch der im November 1999 in der Frankfurter Rundschau veröffentlichte Bericht über die pädophilen Erfahrungen von fünf ehemaligen Schülern durch den damaligen Leiter der Odenwaldschule Gerold Becker führten zu keiner Aufklärung. Das änderte sich 2010. Nach einem, wie er es selbst nennt, »Schlüsselgespräch « (Mertes, 2013, S. 39) mit drei ehemaligen Berliner Jesuitenschülern des Canisius-Kolleg im Januar 2010 schrieb der damalige Rektor Pater Klaus Mertes SJ am 20.01.2010 einen Brief an circa 600 potenzielle Opfer von sexualisierter Gewalt am Berliner Canisius-Kolleg. In diesem Brief entschuldigte er sich stellvertretend für das Kolleg bei den von Missbrauch betroffenen ehemaligen Schülern. Er ahnte damals » nicht, was für eine Lawine dieser Brief auslösen würde« (ebd., S. 19). Dies geschah durch die Veröffentlichung des Briefes durch die Berliner Morgenpost am 28.01.2010 (vgl. ebd., S. 25). In der Folge wurden weitere Fälle aus Kirchen, Schulen und Institutionen der Erziehungshilfe öffentlich. In Deutschland entwickelte sich infolge dessen »ein Diskurs um seit Jahrzehnten stattfindende Misshandlungen und sexuellen Missbrauch in Institutionen « (Fegert et al., 2015, S. 35). Das Thema sexueller Missbrauch rückte in die Mitte der Gesellschaft und wurde auch in den deutschen Massenmedien intensiv debattiert. ${ }^{3}$ Hatten zuvor insbesondere Fraueneinrichtungen sexuelle Gewalt thematisiert, aber kein breiteres gesellschaftliches Echo bekommen, so kam nun eine breitere Diskussion auf und es wurden finanzielle Programme initiiert, um Maßnahmen zur Prävention sexuellen Missbrauchs auf den Weg zu bringen. Das erste Mal kamen betroffene Jungen in den Blick. Diese mediale Präsenz des Themas veränderte die bis dahin geführten Diskussionen und zwang öffentliche Akteure zum Handeln (vgl. ebd., S. 38).

3 Einen guten Bericht über die ersten Monate nach dem Januar 2010 hat die Redaktion der Berliner Morgenpost anlässlich der Verleihung des »Wächterpreises 2011 «am 17.05.2011 veröffentlicht: http://www.journalist.de/aktuelles/ meldungen/eine-unendliche-geschichte-waechterpreis-gewinner-berliner -morgenpost.html (14.05.2016). 
Die Bundesregierung ernannte Christine Bergmann im März 2010 zur ersten Unabhängigen Beauftragten zur Aufarbeitung des sexuellen Kindesmissbrauchs. Zeitgleich richtete sie den Runden Tisch »Sexueller Kindesmissbrauch in Abhängigkeits- und Machtverhältnissen in privaten und öffentlichen Einrichtungen und im familiären Bereich « (folgend: Runder Tisch »Sexueller Kindesmissbrauch «) ein, der von März 2010 bis November 2011 Empfehlungen zur Prävention sexueller Gewalt und zur Unterstützung der von ihr Betroffenen erarbeitete (vgl. Abschlussbericht des Runden Tisches, 2011). Parallel dazu wurden Aufarbeitungs- und Forschungsprozesse angestoßen. Die Ergebnisse der gegründeten Runden Tische »Sexueller Kindesmissbrauch « und $\gg$ Heimerziehung in den 50er und 60er Jahren « (2008 von der Bundesregierung etabliert) sowie Erkenntnisse aus dem Aktionsprogramm »Frühe Hilfen « und anderen Diskussionen mündeten schließlich in das Bundeskinderschutzgesetz, welches am 01.01.2012 in Kraft getreten ist. Das Bundeskinderschutzgesetz ist ein sogenanntes Artikelgesetz und besteht aus sechs Artikeln. In Artikel 2 werden rund 30 Änderungen des SGB VIII beschrieben, um den Kinderschutz zu verbessern (vgl. Kunkel, 2015, Rn. 38, S. 33).

\section{Das SGB VIII - Kinder- und Jugendhilfe}

Die Kinder- und Jugendhilfe in Deutschland und die Zuständigkeitsbereiche der Leistungen im Verhältnis zu den anderen Sozialgesetzbüchern wird im SGB VIII beschrieben. Im Folgenden ein kurzer Überblick:

Das erste Kapitel umfasst mit den $\$ \$ 1-10$ die $\gg$ Allgemeinen Vorschriften «. Sie definieren die Leitorientierung und Grundsätze der Jugendhilfe. Grundlegende Bestimmungen zum Verhältnis zwischen Eltern, Kind und Staat sowie der Zusammenarbeit von öffentlichen und freien Trägern der Jugendhilfe sind darin enthalten. Der $\$ 1$ hat hier zentrale Bedeutung: Aus ihm lässt sich die Zielbestimmung der Jugendhilfe ableiten (vgl. Münder et al., 2012, Rn. 63). Die folgenden Festlegungen sind zentral: Es sollen nach $\$ 1$ Abs. 3 Nr. 1 SGB VIII junge Menschen in ihrer individuellen und sozialen Entwicklung gefördert werden; Benachteiligungen sollen vermieden und abgebaut werden. Die 
Kinder- und Jugendhilfe kann dies durch die Förderung von Kindern in Tageseinrichtungen (\$22a i.V.m. $\$ 22$ SGB VIII), in der Kindertagespflege ( $\$ 23$ i. V.m. \$22 SGB VIII) bzw. auch durch die Jugendarbeit ( $\$ 11$ SGB VIII) erreichen. Der $\$ 9$ Nr. 3 SGB VIII verpflichtet die Jugendhilfe zur Gleichberechtigung der Geschlechter und zum Abbau von Benachteiligungen gegenüber Mädchen.

Das Recht der Eltern auf Erziehung und Pflege des Kindes nach dem Grundgesetz Art. 6 Abs. 2 Satz 1 wird wörtlich in $\$ 1$ Abs. 2 SGB VIII übernommen: »Pflege und Erziehung der Kinder sind das natürliche Recht der Eltern und die zuvörderst ihnen obliegende Pflicht.« Das bedeutet für die öffentliche Jugendhilfe, dass sie keinen eigenen Erziehungsauftrag hat. Gleichwohl untermauert der Absatz 3 des gleichen Paragrafen die Aufgaben der Kinder- und Jugendhilfe, die Erfüllung des Erziehungsanspruchs durch Hilfen für Familien sicherzustellen. Um die Erziehungskompetenzen der Eltern zu stärken, kann die Jugendhilfe ihnen Leistungen zur Förderung der Erziehung in der Familie (\$\$16-21 SGB VIII) und Hilfen zur Erziehung (\$\$27-35 SGB VIII) anbieten. Durch das staatliche Wächteramt müssen sich Eltern fragen lassen, ob das Wohl des Kindes in ihrer Erziehung gewährleistet ist. Denn »das Elternrecht nach dem Grundgesetz geht nur soweit, wie es dem Wohl des Kindes dient. Elternrecht ist als Elternverantwortung Garantie für die Verwirklichung des Kindeswohls« (Kunkel, 2015, Rn. 40, S. 37). Oder wie es Ludwig Salgo schreibt: »[D]as Leben eines Kindes und dessen körperliche Unversehrtheit müssen im Verhältnis zu elterlichen Bestimmungsrechten immer ein höheres Gewicht genießen « (Salgo, 2007, S. 18). Bei Missbrauch des Elternrechts hat der öffentliche Träger einzugreifen, dabei ist der Maßstab das Kindeswohl (vgl. Jung, SGB VIII Einführung, S. 2).

Die »anderen Aufgaben der Jugendhilfe $\ll$ sind im dritten Kapitel des SGB VIII beschrieben: Der $\$ 42$ regelt die Inobhutnahme von Kindern und Jugendlichen, ebenso die Beteiligung des zuständigen Jugendamtes bei gerichtlichen Verfahren wie Vormundschaft, Adoption, Gewaltschutz und Jugendgerichtshilfe. Dies sind Bereiche, die durch das staatliche Wächteramt als weitere Aufgaben und nicht als Leistungen der Jugendhilfe abgeleitet wurden. »Diese Aufgaben werden grundsätzlich von den öffentlichen Trägern wahrgenommen. Freie Träger dürfen mit solchen hoheitlichen Aufgaben nur ausnahmswei- 
se betraut werden (vgl. $\$ 3$ Abs. 3) « (ebd., S. 3). Das darauffolgende vierte Kapitel des SGB VIII legt den Umgang und die Erhebung von Sozialdaten innerhalb der Kinder- und Jugendhilfe dar. Unter Sozialdaten versteht man »Einzelangaben über persönliche oder sachliche Verhältnisse einer bestimmten oder bestimmbaren natürlichen Person « ( $\$ 3$ Abs. 1 Bundesdatenschutzgesetz). Persönliche Verhältnisse sind der Name, Geburtstag, Krankheiten und empfangene Hilfeleistungen. Unter sachlichen Verhältnissen sind unter anderem Telefonnummer und E-Mail-Adresse gemeint.

Zum Beispiel wird im $\$ 65$ Abs. 1 der besondere Vertrauensschutz in der persönlichen und erzieherischen Hilfe beschrieben. Sozialdaten, die zum Zweck persönlicher und erzieherischer Hilfe Mitarbeitenden übergeben wurden, dürfen unter anderem nur weitergegeben werden, wenn eine Einwilligung desjenigen vorliegt, der die Daten anvertraut hat (Nr. 1), wenn es dem Familiengericht zur Erfüllung seiner Aufgaben im Kinderschutz (nach $₫ 8$ a Abs. 2) dient, wenn ohne die Erfüllung dieser Aufgaben die notwendige Entscheidung nicht getroffen werden kann (Nr. 2) oder wenn zur Abschätzung eines Gefährdungsrisikos Fachkräfte hinzugezogen werden (Nr. 4). Diese weitergegebenen anvertrauten Sozialdaten dürfen vom Empfänger nur für den dafür vorgesehenen Zweck verwendet werden. Ebenso ist geregelt, dass Sozialdaten nur erhoben $(\$ 62)$ und gespeichert $(\$ 63)$ werden dürfen, wenn dies für die Erfüllung der Aufgabe notwendig ist.

Die Vorschriften für den Träger der öffentlichen Jugendhilfe werden in den \$\$69-72a näher erläutert. Hier ist für die Arbeit mit ehrenamtlich Tätigen vor allem der $\$ 72$ zum Tätigkeitsausschluss einschlägig vorbestrafter Personen relevant. Wie die Anerkennung, Förderung und Zusammenarbeit mit der freien Jugendhilfe geregelt ist, klären die $\$ \$ 73-78$. Die Gesamtverantwortung der Erfüllung des SGB VIII wird als die der öffentlichen Träger benannt (vgl. \$\$79-81).

Die Kapitel sechs bis acht befassen sich mit den zentralen Aufgaben der Länder und des Bundes, die sachliche, örtliche und finanzielle Zuständigkeit sowie der Kostenerstattung in allen zuvor beschriebenen Bereichen der Kinder- und Jugendhilfe. Das neunte und zehnte Kapitel beinhalten die Regelungen der Kinder- und Jugendhilfestatistik und die Straf- und Bußgeldvorschriften. 


\section{Das Bundeskinderschutzgesetz und seine Auswirkung auf das SGB VIII, fokussiert auf die Jugendverbandsarbeit}

Zum 01.01.2012 trat das »Gesetz zur Stärkung eines aktiven Schutzes von Kindern und Jugendlichen « (das sog. Bundeskinderschutzgesetz BKiSchG) in Kraft. Es enthält sechs Artikel: das Gesetz zur Kooperation und Information im Kinderschutz (KKG - Art. 1), die Änderungen im SGB VIII (Art. 2), die Änderung anderer Gesetze im SGB IX und dem Schwangerschaftskonfliktgesetz (Art. 3), die Pflicht der Evaluation (bis zum 31.12.2015 vor dem Bundestag vorzutragen - Art. 4) ${ }^{4}$, Regelung der Veröffentlichungsermächtigung (Art. 5) und den mit Artikel 6 festgelegten Zeitpunkt des Inkrafttreten des Gesetzes (vgl. Jans et al., Vorbem. Art. 1 BkiSchG [KKG] Rn. 2 [Schimke]). Mit der Einführung des Gesetzes erwartete man eine umfassende Verbesserung des Kinderschutzes.

Die wichtigen Änderungen im SGB VIII sind für die Jugendverbandsarbeit $\$ 8$ a und der neu gefasste $\$ 72$ a SGB VIII.

$>$ Der $\$ 8$ a SGB VIII wurde neu strukturiert und die Aufgaben des Jugendamtes/Trägers der öffentlichen Kinder- und Jugendhilfe und der freien Träger wurden systematisch getrennt. Er beschreibt, wie vorher schon, das Vorgehen bei Kindeswohlgefährdung und definiert die Zuständigkeiten. Neu aufgenommen wurde das fachliche Profil der insoweit erfahrenen Fachkraft (vgl. Anhang A3) als Gegenstand der Vereinbarungen von öffentlichem Träger mit den freien Trägern sowie die Verpflichtung des Jugendamtes zum Hausbesuch bei Erforderlichkeit.

$>$ Der $\$ 72$ a regelt den Tätigkeitsausschluss einschlägig vorbestrafter Personen, neu auch der von ehrenamtlich Tätigen in der Kinder- und Jugendhilfe. Das erweiterte polizeiliche Führungszeugnis dient hierfür als ein Instrument der Überprüfung einer entsprechenden strafrechtlichen Verurteilung.

$>$ Der neue $\$ 8 \mathrm{~b}$ (Fachliche Beratung und Begleitung zum Schutz von Kindern und Jugendlichen) Abs. 1 ist für Personen, die

4 Die Evaluation wurde vom Deutschen Jugendinstitut (DJ) durchgeführt und am 16.12.2015 dem Bundestag vorgestellt. 
im beruflichen Kontext in Kontakt mit Kindern und Jugendlichen sind, relevant. Er beschreibt einen Beratungsanspruch durch Fachberatungsstellen oder den öffentlichen Jugendhilfeträger für diesen Personenkreis im Zusammenhang mit Fragen des Kinderschutzes. Damit ist auch für Personen, die außerhalb der Kinderund Jugendhilfe tätig sind, aber mit Kindern und Jugendlichen in beruflichem Kontakt stehen (z. B. Lehrer_innen, Angestellte im Sportverein oder Musikschulen), die fachliche Beratung bei der Gefährdungseinschätzung geregelt. Wiesner gesteht dies auch ehrenamtlich Tätigen zu, die im Wortlaut des Gesetzes nicht erfasst sind. Ihnen sollte jedoch »eine Beratung auf Wunsch nicht verwehrt werden « (Fegert \& Wolff, 2015, S. 320).

$>\quad$ Neu ist im $\$ 8$ der Abs. 3, der Kindern und Jugendlichen den Zugang zur Beratung ohne Kenntnis der Personensorgeberechtigten zusichert, sofern sie in einer Not- oder Konfliktlage sind und solange eine Mitteilung an den Personensorgeberechtigten dem Beratungszweck entgegenstehen würde. Grundlage für diesen Absatz war die Forderung des Runden Tisches »Sexueller Kindesmissbrauch «, »Kindern und Jugendlichen einen eigenen Anspruch auf Beratung in Krisen- und Konfliktsituationen einzuräumen « (ebd., S. 318).

Im Vorfeld übten die Jugendverbände Kritik an der Umsetzbarkeit der Neuregelung des $\$ 72 \mathrm{a}$ SGB VIII (vgl. Stellungnahmen des Bundesjugendrings, 2008 und 2015). Besonders wird der hohe bürokratische Aufwand für Vereine und Verbände der Jugendarbeit herausgestellt; die Vereine würden meist von jungen ehrenamtlichen Personen geleitet, welche keine Unterstützung einer professionellen Personalverwaltung haben. Hierzu gibt es Vorschläge, die eine Vereinfachung darstellen, jedoch im Gesetzesentwurf keine Beachtung fanden.

Das Projekt »Wirkungen des Bundeskinderschutzgesetzes - Wissenschaftliche Grundlagen « (2013-2015), das vom Deutschen Jugendinstitut (DJI) durchgeführt wurde, untersuchte, ob die gesetzlichen Regelungen die entsprechenden Wirkungen in der Praxis erzielten.

Die Evaluation, in welcher der Frage der Weiterentwicklung der Praxis der Kinder- und Jugendhilfe nach Einführung des Bundeskinderschutzgesetzes nachgegangen wurde, ist sehr umfangreich an Methoden 
und Perspektiven. Die am 16.12.2015 vorgestellten Ergebnisse zeigen positive Entwicklungen: So erreichen die Angebote der Frühen Hilfen über Familienhebammen junge Eltern. Weiter zeigen die Ergebnisse, dass die Vernetzung wichtiger Akteure im Kinderschutz gut funktioniert und auch die Jugendämter gut vernetzt sind. Im Bereich der Kinder- und Jugendhilfe werden nach Angaben des Berichtes jährlich etwa 100 Personen aufgrund der Einsichtnahme in das erweiterte polizeiliche Führungszeugnis von der Tätigkeit ausgeschlossen (vgl. Evaluationsbericht 2015).

Der Deutsche Bundesjugendring bedauert in seiner Stellungnahme zum Bericht der Bundesregierung jedoch, dass Jugendverbände nicht befragt wurden. Sie, als größte Gruppe unter den Trägern von Jugendarbeit, hätten gerade im Bereich der Regelungen zum $\$ 72$ a SGB VIII wichtige Erkenntnisse beitragen können. Weiter merkt er an, dass die Befragung von Jugendringen aufgrund derer $\gg$ Spezifika [...] nur sehr begrenzt auf Jugend- oder Sportverbände « übertragbar sind (BJR, 2016, S. 1).

Manuela Schwesig, die Bundesfamilienministerin, verkündete nach dem Bericht im Bundestag, dass sie » mit einer Gesamtreform der Kinder- und Jugendhilfe das Kind und seine Bedürfnisse noch stärker in den Fokus rücken « möchte (BMFSFJ, 16.12.2015). Damit kündigte sie gleichzeitig eine Gesamtreform der Kinder- und Jugendhilfe an.

\section{Der §8a SGB VIII - Schutzauftrag bei Kindeswohlgefährdung}

Mit der Einführung des $\$ 8$ a im SGB VIII 2005 wurde dem Schutz des gefährdeten Kindes durch ein geregeltes Verfahren bei Hinweisen auf eine Gefährdung ein hoher Stellenwert eingeräumt. Das Ziel der Kinder- und Jugendhilfe ist der Schutz vor Gefahren. Dieser Schutz kann unter Umständen auch mit Eingriffen in die Grundrechte der Beteiligten verbunden sein (vgl. Art. 6, Art. 1 oder Art. 2 GG). Die Ausgestaltung der Schutzverpflichtung ist Aufgabe des Gesetzgebers.

Die Stellung des $\$ 8$ a im ersten Kapitel des SGB VIII spiegelt die Wichtigkeit wider, die dem Kinderschutz zukommt. Er gehört zum Grundsatz der Jugendhilfe, nicht nur zu deren Aufgaben. Mit dem §8a 
wird das Thema Kinderschutz nicht abschließend behandelt - er beschäftigt sich spezifisch mit der Situation, in der es Anhaltspunkte für eine Gefährdung des Wohls von Kindern und Jugendlichen gibt, und beschreibt das Vorgehen in einem solchen Fall. Kunkel beschrieb ihn in einem Kehler Diskussionspapier vor der Einführung des Bundeskinderschutzgesetzes als »Fahrplan « für das Jugendamt bei Verdacht auf Kindeswohlgefährdung (vgl. Kunkel, 2006, S. 3). Auch nach der Einführung des Bundeskinderschutzgesetzes ist dies so geblieben, jedoch haben sich die Verantwortung und Aufgaben der freien Träger verändert. Im Absatz 1 werden die Aufgaben des Jugendamtes bei Bekanntwerden gewichtiger Anhaltspunkte für die Gefährdung des Wohls eines Kindes oder Jugendlichen beschrieben. Dieses Verfahren ist dreistufig und lautet wie folgt:

I. Erkennen von Anhaltspunkten

II. Bewerten des Gefährdungsrisikos (in Zusammenarbeit von Fachkräften, Eltern und Kindern)

III. Handeln zur Abwehr der Gefährdung (Gewährung von Hilfen)

Im zweiten Abschnitt des $\$ 8$ a wird auf den $\$ 1666$ BGB verwiesen. Darin ist geregelt, dass das Jugendamt erst dann das Familiengericht anrufen kann, wenn die Eltern bzw. Personensorge- oder Erziehungsberechtigten bei der Abwendung der Gefahr nicht ausreichend mitgewirkt haben und das körperliche, geistige oder seelische Wohl des Kindes oder Jugendlichen weiterhin gefährdet ist. Erst dann entscheidet das Familiengericht über das weitere Vorgehen.

Im dritten Abschnitt wird die Art und Weise der Hinzuziehung anderer Leistungsträger, der Einrichtungen der Gesundheitshilfe und der Polizei geregelt.

Für freie und private Träger der Jugendhilfe wird dieser Paragraf dann relevant, wenn sie mit dem öffentlichen Träger (Jugendamt) Vereinbarungen über zu erbringende Leistungen abschließen. Diese Vereinbarungen werden mit dem Abs. 4 Satz 1 des $\$ 8$ a geregelt und für die Jugendämter als Pflicht auferlegt. Die Fachkräfte in Einrichtungen und Diensten haben mit Kindern und Jugendlichen spezielle Erfahrungen und gute Beobachtungsmöglichkeiten, um den Kinderschutz wahrzunehmen. »Sie merken häufig unmittelbar, wenn das Wohl eines Kindes oder Jugendlichen bedroht ist« (Jans et al., \$8a Rn. 55 [Har- 
nach]). Im weiteren Verlauf des Absatzes wird erläutert, wie sich der Träger zu verhalten hat:

$>$ Sicherstellung, dass die Fachkräfte den Schutzauftrag nach Absatz 1 wahrnehmen

$>$ Hinzuziehen einer insoweit erfahrenen Fachkraft bei der Abschätzung des Gefährdungsrisikos

$>$ Einbeziehen derErziehungsberechtigten sowie des Kindes/des_der Jugendlichen in die Gefährdungseinschätzung, sofern es nicht dem wirksamen Schutz des Kindes/des_der Jugendlichen entgegensteht

$>$ Hinwirken, dass der Erziehungsberechtigte Hilfen in Anspruch nimmt

$>$ Gegebenenfalls Information an das Jugendamt, wenn Hilfen nicht ausreichen oder die Erziehungsberechtigten diese Hilfen nicht annehmen und eine Gefährdung des Kindes/des_der Jugendlichen nicht auszuschließen ist

Mit einer Vereinbarung zur Sicherstellung des Schutzauftrags zwischen öffentlichem und freiem Träger wird der Schutzauftrag somit auch auf Fachkräfte (nicht auf die ehrenamtlichen Personen) von Trägern der freien Jugendhilfe übertragen. Das Team muss bei einer Abschätzung eine Fachkraft von außen hinzuziehen, deren Qualifikation in den Vereinbarungen aufgenommen werden soll. Im Unterschied zum öffentlichen Träger haben freie Träger keine hoheitlichen Aufgaben zu erfüllen, sind nicht zum Hausbesuch verpflichtet und dürfen sich bei Dritten auch keine weiteren Informationen beschaffen (vgl. ebd.).

Im Abschnitt 5 ist die Weitergabe von Daten zur Gewährung von Leistungen geregelt.

\section{Der §72a SGB VIII - Tätigkeitsausschluss einschlägig vorbestrafter Personen}

Ein für die verbandliche Jugendarbeit weitreichender und mit vielen Diskussionen behafteter Paragraf des SGB VIII ist der $\$ 72$ a. Er regelt den Tätigkeitsausschluss einschlägig vorbestrafter und damit ungeeigneter Personen von der Betreuung, Erziehung und Ausbildung in der Kinder- und Jugendhilfe durch die Vorlage bzw. die Einsichtnahme in 
erweiterte Führungszeugnisse. »Damit soll dem besonderen Schutzbedürfnis von Kindern und Jugendlichen in Bezug auf Sexualstraftaten Rechnung getragen und berücksichtigt werden, dass ansonsten möglichen Tätern Zugang außerhalb des elterlichen Einflussbereichs eröffnet würde « (Jung, §72a Rn. 2 SGB VIII). Einen Einblick in die Diskussionen bezüglich der Einsichtnahme bei ehrenamtlich Mitarbeitenden in der verbandlichen Jugendarbeit erfolgt in Kapitel 4 dieses Bandes.

Im ersten und zweiten Abschnitt des $\$ 72 \mathrm{a}$ sind die Regelungen bezüglich eines Tätigkeitsausschlusses genannt, in Absatz 1 für beruflich Beschäftigte bei öffentlichen Trägern in der Kinder- und Jugendhilfe und in Absatz 2 über Vereinbarungen für freie Träger und deren Beschäftigte. So muss die Vorlage eines Führungszeugnisses vor Beginn der Tätigkeitsaufnahme erfolgen. Träger dürfen keine Personen beschäftigen, die wegen eines Straftatbestands rechtskräftig verurteilt wurden. Im Absatz 1 werden diese Straftaten des Strafgesetzbuchs (StGB) aufgeführt: $\$ \$ 171,174-174 c, 176-180 a, 181 a, 182-184 \mathrm{~g}, 225,232-233 a$, 234, 235 und 236 des Strafgesetzbuches; es handelt sich also um Straftaten gegen die sexuelle Selbstbestimmung (siehe Anhang A2).

Der dritte Abschnitt beschreibt die Verantwortung der öffentlichen Jugendhilfe, keine neben- oder ehrenamtlich tätigen Personen zu beschäftigen, die nach den im Absatz 1 genannten Straftaten verurteilt wurden. Die Entscheidung einer Einsichtnahme in das Führungszeugnis vor der Aufnahme der ehrenamtlichen Tätigkeit soll auf Grundlage der Tätigkeitsbeschreibung erfolgen. Hierbei ist Art, Intensität und Dauer des Kontaktes mit Kindern und Jugendlichen zu berücksichtigen.

Für die verbandliche Jugendarbeit ist der vierte Abschnitt wichtig. Es ist in ihm die Regelung für neben- und ehrenamtlich tätige Personen in der Arbeit der Träger der freien Jugendhilfe und von Vereinen im Sinne des $\$ 54$ (Vereinsvormundschaften) beschrieben. Der Absatz regelt die Verpflichtung des öffentlichen Trägers, in Vereinbarungen mit dem öffentlichen Träger sicherzustellen, dass unter der Verantwortung der Träger der freien Jugendhilfe keine Person neben- oder ehrenamtlich »Kinder oder Jugendliche beaufsichtigt, betreut, erzieht oder ausbildet oder einen vergleichbaren Kontakt hat «. Über die entsprechenden Tätigkeiten und Möglichkeiten des Aufbaus eines besonderen Vertrauensverhältnisses soll es eine Vereinbarung geben, die auf der Grundlage 
von Art, Intensität und Dauer des Kontaktes die Einsichtnahme in ein Führungszeugnis erforderlich macht.

Im Gegensatz zu den Regelungen bezüglich hauptamtlicher Personen ist hier von einer Vorlage die Rede. Es geht dem Gesetzgeber nicht um eine allgemeine Vorlagepflicht, sondern um eine Differenzierung nach Tätigkeiten. Dafür gibt er das Instrument der Vereinbarung vor, in der die entsprechenden Tätigkeiten nach »Art, Intensität und Dauer « (vgl. \$72a Abs. 4 SGB VIII) des Kontaktes mit dem Kind und der Möglichkeit des Vertrauensaufbaus bewertet werden soll. Die vor der Verabschiedung des Bundeskinderschutzes geführte kontroverse Debatte hat dazu geführt, dass der Gesetzgeber hier » von einer einheitlich gesetzlichen Regelung abgesehen und die Lösung einer Vereinbarung zwischen dem örtlichen Träger der Jugendhilfe und den freien Träger überlassen [hat] «(Fegert \& Wolff, 2015, S. 321). Wiesner weist auf die Nachteile dieser Regelung hin: einen hohen Aufwand und überregional unterschiedliche Handhabungen der Gesetzeslage (vgl. ebd., S. 322).

Dass im Rahmen der Überprüfung über Führungszeugnisse nur bestimmte Daten dokumentiert werden dürfen, regelt der fünfte $\mathrm{Ab}$ schnitt. Hier gilt: Es darf nur dokumentiert werden,

$>$ dass die Einsichtnahme in das erweiterte Führungszeugnis erfolgt ist,

$>\quad$ das Datum des Führungszeugnisses,

$>$ die Information, ob die betreffende Person wegen einer Straftat nach Abs. 1 Satz 1 rechtskräftig verurteilt wurde.

Diese Daten dürfen nur gespeichert werden, wenn es für den Ausschluss der Person von der Tätigkeit erforderlich ist. Die Daten sind vor dem Zugriff Dritter zu schützen.

Die Löschung der Daten hat zu erfolgen, und zwar

$>$ unverzüglich, wenn im Anschluss an die Einsichtnahme keine Tätigkeit wahrgenommen wird, bzw.

$>\quad$ spätestens drei Monate nach der Beendigung der Tätigkeit.

Für die Beantragung sind die $\$ \$ 30$ und 30 a des Bundeszentralregistergesetzes (BZRG) relevant. 


\section{Bedeutung zentraler Begriffe}

Im Folgenden werden zentrale Begriffe geklärt, die zum Verständnis der rechtlichen Regelungen beitragen und in Verbindung mit Schulungen für Ehrenamtliche von Bedeutung sind. Erstaunlich ist, dass einige wichtige Begriffe auch gesetzlich nicht definiert sind und sich so die Fachöffentlichkeit eine Definition erarbeiten muss(te).

\section{Sexualisierte Gewalt}

Kindeswohlgefährdung, Kindesmissbrauch, sexueller Übergriff, sexueller Missbrauch, sexuelle Ausbeutung, Misshandlung, sexualisierte Gewalt, sexuell grenzverletzendes Verhalten ... (für eine genauere Analyse der Termini in der Fachliteratur vgl. Wipplinger \& Amman, 2005, S. 14-43) - diese und noch mehr Begriffe finden sich für ein Phänomen: sexualisierte Gewalt.

Im Alltag und der wissenschaftlichen Literatur existiert eine Vielzahl an Begriffen für diesen einen Tatbestand. Häufig wird der Begriff »sexueller Missbrauch « verwendet. Diesen findet man im Sprachgebrauch von Wissenschaftler_innen, dem Gesetzgeber und in den Medien. Damit ist er im öffentlichen Sprachgebrauch präsent. Allerdings wird er auch von Fachkräften infrage gestellt, vor allem weil er impliziert, dass eine Person eine andere »gebrauchen « kann - im Sinne von »nutzen « - und nur der fälschliche $\gg$ Gebrauch « als Missbrauch problematisch sei. Daher wird fachlich der Terminus » sexuelle Gewalt « bevorzugt verwendet. 
»Diese Formulierung stellt heraus, dass es sich um Gewalt handelt, die mit sexuellen Mitteln ausgeübt wird. Der ebenfalls verwendete Begriff > sexualisierte Gewalt< geht noch einen Schritt weiter und verdeutlicht, dass bei den Taten Sexualität funktionalisiert, also benutzt wird, um Gewalt auszuüben « (UBSKM, 2016).

Hier liegt der Fokus also auf der Ausnutzung eines Macht- und Abhängigkeitsgefälles, um eigene Bedürfnisse auf Kosten des Kindes/des_der Jugendlichen zu befriedigen bzw. Kinder oder Jugendliche zu demütigen, herabzusetzen oder zu verletzen. Dazu gehört jedes Verhalten, das die sexuelle Selbstbestimmung und persönliche Integrität eines anderen Menschen beschneidet - hier speziell fokussiert auf Kinder und Jugendliche: sexuelle Belästigung im Alltag, sexistische Sprache und Bedrohungen, aufgedrängte Zärtlichkeiten und Berührungen, erzwungene sexuelle Handlungen, sexueller Missbrauch und auch Vergewaltigung.

Wichtig für den Bereich der verbandlichen Jugendarbeit ist das Bewusstsein, dass es im Themenbereich der Prävention sexualisierter Gewalt nicht nur um die Sensibilisierung von ehren- und hauptamtlichen Personen geht, sondern dass auch sexualisierte Gewalt unter Kindern und Jugendlichen im Blick ist und hier eine deutliche Stellungnahme erfolgt.

Wird im institutionellen, verbandlichen oder kirchlichen Bereich von »sexualisierter Gewalt « gesprochen, so schließt das neben den strafrechtlich relevanten Formen von sexueller Gewalt auch die Bereiche mit ein, die sich im rechtlichen Graubereich befinden, aber im seelsorgerlichen oder erzieherischen sowie im betreuenden oder pflegerischen Umgang mit Kindern, Jugendlichen und Erwachsenen eine Grenzüberschreitung darstellen. Grenzüberschreitungen werden subjektiv empfunden und können objektiv nicht wahrgenommen oder bewertet werden. So kann eine Umarmung als grenzverletzend, aber auch als tröstend empfunden werden. Schamgrenzen können durch ungeklärte Umkleide- oder Waschsituationen auf Ferienlagern verletzt werden.

Daher ist, besonders wenn es um Prävention sexualisierter Gewalt geht, eine Unterscheidung innerhalb des Begriffes sinnvoll. In der pädagogischen und sozialarbeiterischen Praxis helfen diese Unterscheidungen bei der Auswahl und Entwicklung angemessener Präventionsmaßnahmen. 
In der Fachpraxis wird dabei folgende Einteilung nach Schweregrad vorgenommen (vgl. Enders, 2010; 2012):

$>$ Grenzverletzung

> (sexueller) Übergriff

$>$ Strafrechtlich relevante Formen

\section{Grenzverletzungen}

Grenzverletzungen treten einmalig oder gelegentlich im (pädagogischen) Alltag auf. Diese Grenzverletzungen geschehen in der Regel unabsichtlich, können aber auch einen Hinweis auf persönliche oder fachliche Mängel von Mitarbeitenden sein. Grenzverletzungen sind zum Beispiel die Missachtung persönlicher oder körperlicher Grenzen oder der Grenzen der professionellen Rolle. Dazu gehören das Aufdrängen intimer Nähe, das Verletzen der Schamgrenze, das Gespräch über sexuelle Erlebnisse oder auch anzügliche Kommentare. Grenzverletzungen sind subjektive Empfindungen und nicht objektiv bewertbar. Diese Verhaltensweisen sind durch fachliche Anleitung, klare Regeln, achtsamen, respektvollen und sensiblen Umgang mit einem Nähe-DistanzVerhältnis und Entschuldigungen korrigierbar. Werden grenzverletzende Verhaltensweisen nicht korrigiert und thematisiert, können sie auch eine Vorstufe von sexualisierter Gewalt darstellen und als übergriffiges Verhalten empfunden werden.

\section{(Sexuelle) Übergriffe}

Übergriffiges (sexualisiertes) Verhalten geschieht in der Regel massiv, nicht versehentlich und ist immer ein persönliches Fehlverhalten. Dazu gehören wiederholte Missachtung der professionellen Rolle und die Missachtung der Grenzen zwischen den Generationen, sexistische Spielanleitungen bei Programmen der Jugendarbeit, wiederholte, vermeintlich zufällige Missachtung persönlicher und körperlicher Grenzen oder auch das bewusste Ängstigen von Kindern und Jugendlichen durch Angst machende Rituale oder Spiele. Abwehrende Reaktionen und auch Kritik von Dritten wird missachtet. Ein solches Verhalten ist nicht entschuldbar und erfordert eine klare Stellungnahme mit Konsequenzen vonseiten der Leitung. 


\section{Strafrechtlich relevante Gewalthandlungen}

Strafrechtlich relevante Gewalthandlungen umfassen neben den Tatbeständen der Körperverletzung auch die im Strafgesetzbuch als »Straftaten gegen die sexuelle Selbstbestimmung « bezeichneten Handlungen. Sie sind im 13. Abschnitt des Strafgesetzbuches benannt (siehe auch Anhang A2). Diese Handlungen können mit und ohne Körperkontakt stattfinden. So sind strafbare Handlungen ohne Körperkontakt zum Beispiel exhibitionistische Handlungen, die Förderung sexueller Tätigkeiten zwischen Minderjährigen sowie die Herstellung und Verbreitung von pornografischen Produkten.

\section{Fachkraft und Eignung nach $\$ 72$ SGB VIII}

Maßgeblich für die Definition einer Fachkraft ist $\$ 72$ SGB VIII. Demnach sind Fachkräfte Personen, die eine der Aufgabe entsprechende qualifizierte Ausbildung erhalten haben (vgl. \$72 Abs. 1 SGB VIII). Im $\$ 8$ a Abs. 4 wird die » insoweit erfahrene Fachkraft «, die bei einer Risikoabschätzung hinzugezogen werden soll, erwähnt.

Der Begriff der » insoweit erfahrenen Fachkraft « ist bereits im Jahr 2005 durch §8a SGB VIII eingeführt worden. Im Vorfeld der Einführung des Bundeskinderschutzgesetzes wurde über diesen Begriff heftig in der Fachöffentlichkeit diskutiert. Der ins Feld geworfene Begriff »Kinderschutzfachkraft « hat jedoch in den Kreisen der Fachwelt heftigen Widerstand ausgelöst, da die Befürchtung bestand, dass ein neues Berufsbild innerhalb der Sozialen Arbeit geschaffen würde; er wurde daraufhin wieder zurückgenommen (vgl. Jans, [KKG] BKiSchG Art. 1 $\$ 4$ Rn. 17).

Die Wahrnehmung des Schutzauftrages ist nur für beruflich beschäftigte Fachkräfte verpflichtend. Davon ausgenommen sind Laien und Studierende; Praktikant_innen hingegen gelten als Fachkräfte. In den Vereinbarungen zwischen öffentlichen und freien Träger der Jugendhilfe ist festgelegt, wann Fachkräfte als geeignet gelten. Für die Aufgabe einer »insoweit erfahrenen Fachkraft « sind in Fachdiskussionen Kriterien entwickelt worden. Die Empfehlung des bayerischen Landesjugendamtes zur Umsetzung des Schutzauftrages enthält bei- 
spielsweise ein Anforderungsprofil für »insoweit erfahrene Fachkräfte $\ll$. Demnach muss

» mindestens eine der beteiligten Fachkräfte (>erfahrene Fachkraft<) bei der Abschätzung des Gefährdungsrisikos über folgende Qualifikationen verfügen:

- $\quad$ einschlägige Berufsausbildung (z. B. Dipl.-Sozialpäd., Dipl.-Psych., Arzt oder Ärztin)

- Qualifizierung durch nachgewiesene Fortbildung,

- Praxiserfahrung im Umgang mit traumatisierten Kindern und Problemfamilien,

- Fähigkeit zur Kooperation mit den Fachkräften öffentlicher und freier Träger der Jugendhilfe, sowie mit weiteren Einrichtungen, z. B. der Gesundheitshilfe, Polizei ...

- Kompetenz zur kollegialen Beratung; nach Möglichkeit supervisiorische oder Coaching-Kompetenzen,

- persönliche Eignung(z. B. Belastbarkeit, professionelle Distanz, Urteilsfähigkeit $)_{\ll}($ BLJA, 2012, 2.2).

Das Kinderschutzzentrum Berlin hat diese Anforderungen sehr weit ausdifferenziert. Für die Zielführung dieses Buches sind sie jedoch nicht relevant.

Kompetenzen, über die eine Fachkraft verfügen muss, um als »insoweit erfahrene Fachkraft « tätig zu sein, werden auch in der Vereinbarung mit dem Jugendamt Stuttgart beschrieben. Demnach sollte die Fachkraft in gewisser Regelmäßigkeit mit Kinderschutzfragen konkret befasst (erfahren) sein. Als erforderliche Kompetenzen werden unter anderem Kenntnisse über Familiensysteme und die Dynamik konfliktbehafteter Beziehungen, die Kenntnis über Grundbedürfnisse von Kindern und Jugendlichen in den jeweiligen Entwicklungsphasen, Wissen über das Hilfesystem und die Kooperationswege (siehe Anhang A3) angesehen.

Im Gesetzestext finden sich hinsichtlich der Qualifikation von Fachkräften oder »insoweit erfahrenen Fachkräften « keine weiteren genaueren Regelungen. 


\section{Ehrenamtlich Tätige in der verbandlichen Jugendarbeit}

Die Arbeit der verbandlichen Jugendarbeit ist im Wesentlichen auf ehrenamtlich Mitarbeitende angewiesen. Im traditionellen Sinn ist das Ehrenamt ein öffentliches Amt, für das kein Gehalt, aber eine Aufwandsentschädigung gezahlt werden kann. Heute verstehen wir unter Ehrenamt ein freiwilliges Handeln im gemeinnützigen Bereich und zunehmend auch Freiwilligenarbeit, bürgerschaftliches Engagement oder zivilgesellschaftliches Engagement. Eine gesetzliche Definition von Ehrenamt gibt es nicht. Die Begriffe werden meist synonym verwendet. In der verbandlichen Jugendarbeit ist der Begriff des Ehrenamtes jedoch weit verbreitet und bildet »die deutlichste Abgrenzung zu den bezahlten hauptberuflichen oder hauptamtlichen Tätigkeiten « (Fegert \& Wolff, 2015, S. 187).

Das ehrenamtliche, freiwillige Engagement in den Jugendverbänden ist vom Strukturprinzip der Selbstorganisation (vgl. $\$ 11$ Abs. 2 SGB VIII) und der Handlungsmaxime der Interessenvertretung (vgl. $\$ 12$ Abs. 2 SGB VIII) geprägt.

Schaut man sich die dargestellten Kriterien und erforderlichen Qualifikationen für Fachkräfte an, erkennt man, dass ehrenamtlich Mitarbeitende diesen Anforderungen in der Regel nicht entsprechen. Die alleinige Ausbildung von ehrenamtlichen Kräften durch die Jugend-Leiter-Schulungen (JuLeiCa) macht diese nicht zu Fachkräften im Sinne des Gesetzes. Hat ein_e ehrenamtlich Mitarbeitende_r aber eine pädagogische Ausbildung oder ein Studium absolviert, so ist diese Person Fachkraft im Sinne des Gesetzes. Sollte sie aufgrund ihrer Ausbildung als ehrenamtliche Kraft im Kinder- und Jugendverband beschäftigt sein, so unterliegt sie ebenfalls den gesetzlichen Bestimmungen. Damit nicht jede Fachkraft für sich tätig wird und Ehrenamtliche nicht überfordert werden, ist es notwendig, dass es innerverbandliche Regelungen gibt. Ehrenamtliche dürfen nicht mit hauptberuflichen Fachkräften gleichgesetzt werden.

\section{Kindeswohl}

Obwohl »Kindeswohl « in der Fachliteratur ein häufig verwendeter Begriff ist, findet sich an keiner Stelle im Gesetzestext eine Definition. 
Juristisch ist er somit ein sogenannter unbestimmter Rechtsbegriff, der im Einzelfall einer Interpretation bedarf. Das Wohl des Kindes ist immer subjektiv in der jeweiligen Situation zu definieren. Denn »ein am Wohl des Kindes ausgerichtetes Handeln ist dasjenige, welches die an den Grundrechten und Grundbedürfnissen von Kindern orientierte, für das Kind jeweils günstigste Handlungsalternative wählt « (Maywald, 2014, S. 16).

Bei der Annäherung an den Begriff sind die Bedürfnisse von Kindern im jeweiligen Lebensalter hilfreich. Auch die UN-Kinderrechtskonvention kann bei der Definition des individuellen Wohls des Kindes helfen. Sicher spielt auch das Verständnis von Erziehung eine Rolle, denn nach wie vor gibt es keinen umfassenden und für alle gesellschaftlichen Gruppen eindeutigen Konsens darüber, was für das Aufwachsen von Kindern und Jugendlichen das Förderlichste ist. Es gibt hier vielfältige kulturelle, ethnische und zeitgenössische Faktoren. Friederike Alle verweist dazu auf die »Berücksichtigung der Bedürfnisse des Kindes nach der Bedürfnispyramide nach Schmidtchen (1989) « (Alle, 2010, S. 11). Gemeint sind unter anderem die Bedürfnisse nach Geborgenheit, Liebe, angemessener Versorgung, Orientierung und Zuverlässigkeit.

Entwicklungspsychologische Erkenntnisse, individuelles Erleben und das Wissen um die Bedürfnisse von Kindern und Jugendlichen im jeweiligen Lebensalter sind hilfreich für die Definition des Kindeswohls im jeweils aktuell vorliegenden Fall.

\section{Kindeswohlgefährdung}

Nachdem eine eindeutige Definition des Begriffs »Kindeswohl « nicht existiert, könnten wir den Schluss ziehen, dass auch eine klare Definition von Kindeswohlgefährdung nicht zu finden ist. Mithilfe des $\$ 1666$ BGB kann allerdings eine Annäherung und Begriffsbestimmung erfolgen. $\gg$ Der Begriff > Gefährdung des Kindeswohls < stammt ursprünglich aus dem Kindschaftsrecht des BGB « (Alle, 2010, S. 11). Die Eingriffsschwelle, wann das Familiengericht tätig werden muss, wird im $\$ 1666$ Abs. 1 BGB definiert. Kindeswohlgefährdung ist demnach, wenn Informationen über Handlungen gegen Kinder oder Jugendliche oder deren Lebensumstände vorliegen, die das leibliche, geistige oder seeli- 
sche Wohl gefährden. Dabei ist es nicht entscheidend, ob dies durch missbräuchliche Ausübung der elterlichen Sorge, Vernachlässigung des Kindes oder unverschuldetes Versagen der Erziehungsberechtigten oder eines Dritten geschieht. Genauer werden die in Betracht kommenden Gefährdungshandlungen jedoch nicht beschrieben (vgl. Jung, $\$ 8 \mathrm{a}$ Rn. 5).

Eine Rechtsprechung des OLG Zweibrücken (Beschluss vom 03.12.2010 - 2 UF 59/10) besagt, dass eine Kindeswohlgefährdung in Sinne des $\$ 1666$ Abs. 1 Satz 1 BGB vorliegt, » wenn eine gegenwärtige oder zumindest unmittelbar bevorstehende Gefahr für die Kindesentwicklung abzusehen ist, die bei ihrer Fortdauer eine erhebliche Schädigung des körperlichen, geistigen oder seelischen Wohls des Kindes mit ziemlicher Sicherheit voraussehen lässt « (Kunkel, 2015, S. 474).

Sozialwissenschaftlich lassen sich die Fälle von Kindeswohlgefährdung in drei Kategorien einteilen:

> Vernachlässigung (körperliche, emotionale oder erzieherische Vernachlässigung)

$>$ Misshandlung (psychische oder physische)

$>$ sexueller Missbrauch

\section{Formen der Kindeswohlgefährdung und mögliche Signale}

Eine zentrale Frage von Heinz Kindler bei seiner Expertise ist: »[W]ie zuverlässig werden von Kindeswohlgefährdung betroffene Kinder als solche erkannt, so dass seitens der Jugendhilfe bzw. der Familiengerichtsbarkeit Hilfe- und Schutzmaßnahmen eingeleitet werden können?« (Kindler, 2007, S. 5) Er stellt fest, dass »in der Bundesrepublik keine oder kaum Informationen mit zentraler Bedeutsamkeit für die Beurteilung der Ergebnisqualität des Kinderschutzes vorliegen « (ebd., S. 6). Ebenso liegen keine Zahlen über die Anzahl der von der Jugendhilfe bekannt gewordenen Fälle von Kindeswohlgefährdung vor. Daher kann auch nicht gesagt werden, wie zuverlässig Anzeichen von Kindeswohlgefährdung als solche erkannt werden.

In der Literatur finden wir ebenfalls eine Vielzahl von Definitionen bezüglich der Formen von Kindeswohlgefährdung. Häufig werden 
diese sehr feingliedrig ausgeführt. Ich möchte mich bei meinen Ausführungen auf die Kategorisierung von Kindeswohlgefährdungen von Johannes Münder, Barbara Mutke und Reinhold Schone beziehen. Sie unterscheiden vier zentrale Formen:

\section{$>$ Vernachlässigung \\ $>\quad$ körperliche Kindesmisshandlung \\ $>$ sexueller Missbrauch \\ > seelische Kindesmisshandlung}

Hinzu kommen weitere Konfliktfelder aus dem näheren Umfeld, die keine unmittelbare Gefahr darstellen, jedoch durch Zuspitzungen und verstärkende Faktoren für die Gefährdung des Kindeswohls relevant werden. So gehören nach Münder und Kolleg_innen die »Autonomiekonflikte « junger Menschen und »Erwachsenenkonflikte um das Kind « als weitere Kategorie dazu (vgl. Münder et al., 2000, S. 47).

Bei Krieger und Kolleg_innen findet sich eine weitere Differenzierung von Kindesmisshandlung in den oben genannten Kategorien. Sie sprechen von aktiver und passiver sowie psychischer und physischer Vernachlässigung. Das seltene »Münchhausen-by-proxy-Syndrom «bezeichnen sie als eine Sonderform von körperlicher Misshandlung, da dem Kind bewusst (meist durch den Erziehungsberechtigten) körperlicher Schaden zugefügt wird, um Krankheitssymptome vorzutäuschen. Da diese Form der Gewalt eher gegenüber Kleinkindern auftritt, ist sie für die weiteren Ausführungen an dieser Stelle nicht relevant und wird im Folgenden nicht weiter thematisiert.

Ich möchte mich im Folgenden auf die oben genannten Kategorien beschränken, da sie meines Erachtens die wichtigsten im Zusammenhang mit dem Schutzauftrag in der verbandlichen Jugendarbeit sind. Ich möchte die Bezeichnung der Formen der Kindeswohlgefährdung sprachlich dahin gehend gestalten, dass von Vernachlässigung, psychischer und physischer Gewalt sowie von sexuellem Missbrauch gesprochen wird.

In der Praxis wird $\gg$ neben diesen Definitionen unterschieden nach der Häufigkeit, dem Schweregrad, der zeitlichen Dauer sowie dem Alter und Geschlecht der Kinder oder Jugendlichen « (Krieger et al., 2007, S. 13). Darauf möchte ich nicht näher eingehen. Wichtig in diesem $\mathrm{Zu}$ sammenhang ist zu wissen, dass Gewalt gegen Kinder und Jugendliche 
in der Regel keine einmalige Handlung ist, sondern »ein aus mehreren Elementen zusammengesetztes Syndrom negativer Einwirkungen (Handlungen und Unterlassungen) auf das Kind « (Maywald, 2014, S. 22). Zwischen den Misshandlungsformen gibt es einen Zusammenhang, der »logisch « oder auch »funktional « ist. Das ist dann der Fall, wenn Misshandlungsformen eingesetzt werden, um andere Misshandlungsformen zu ermöglichen oder zu decken (vgl. Krieger et. al., 2007, S. 14).

Folgen von Vernachlässigung und Gewalterfahrungen sind nicht klar zuordenbar und es gibt dafür keine klaren Indizien. Franz Moggi beschreibt geschlechtstypische Unterschiede in den Signalen. Männliche und weibliche Opfer von sexualisierter Gewalt leiden so nach einer Studie von David Finkelhor gleich häufig unter den Kurz- und Langzeitfolgen, jedoch zeigen Jungen eher externalisierende und Mädchen eher internalisierende Kurzzeitfolgen (vgl. Moggi, 2005, S. 98).

\section{Vernachlässigung}

Je nach Alter variieren Kriterien für Vernachlässigung. Im Kleinkindalter kann sich eine Vernachlässigung dahin gehend äußern, dass Eltern nicht wissen, womit ihr Kind gerade spielt. Im Schulkindalter wäre ein Indiz für Vernachlässigung, dass die Eltern die beste Freundin ihrer Tochter nicht kennen und nicht wissen, wo und mit wem sie sich gerade aufhält.

Wichtig bei der Definition der Vernachlässigung ist die Kenntnis über altersgerechte Bedürfnisse von Kindern und Jugendlichen. In der Regel ist Vernachlässigung an Unterlassungen in der Beaufsichtigung und Betreuung der Kinder, im Mangel an Kontakt und Unterstützung bei der Befriedigung grundlegender Bedürfnisse und gesundheitlicher Bedarfe zu erkennen. Aber auch die mangelnde Bereitschaft, Gefahren vom Kind abzuwenden, kann ein Hinweis für Vernachlässigung sein. Das Erkennen und Beschreiben von Vernachlässigung bedarf in der Regel einer längeren Zeit und mehr Kontakt. Je jünger das Kind, desto größer ist die Gefahr der Vernachlässigung. Vernachlässigung gehört zu der häufigsten Form von Kindeswohlgefährdung. Kindler beschreibt Vernachlässigung im Handbuch für Kindeswoblgefährdung nach $\$ 1666$ $B G B$ als einen »schleichenden Verlauf mit sich erst allmählich aus- 
bauenden Beeinträchtigungen der kindlichen Entwicklung « (Kindler et al., 2006, S. 41f.). In den ersten Lebensjahren kann aber eine Vernachlässigung dessen ungeachtet rasch zu einem lebensbedrohenden Zustand werden.

Vernachlässigung wird von Krieger und Kolleg_innen in verschiedene Kategorien unterschieden. Sie beschreiben einen Unterschied zwischen physischer und psychischer sowie aktiver und passiver Vernachlässigung (vgl. Krieger et al., 2007, S. 16f.). Auch Kindler gliedert die Formen der Vernachlässigung in verschiedene Bereiche (vgl. Kindler et al., 2006, S. 40ff.).

Demnach wird als physische Vernachlässigung bezeichnet, wenn die Versorgung des Kindes durch unzureichende Ernährung, Pflege und hygienische Mängel gekennzeichnet ist. Die Unterlassung von Sicherheitsmaßnahmen und medizinischer Hilfe und der Mangel an Wohnraum lässt sich ebenfalls als Form von physischer Vernachlässigung beschreiben.

Psychische Vernachlässigung ist gekennzeichnet durch unzureichende emotionale Zuwendung und Aufmerksamkeit, fehlende Bindungsangebote, mangelnde Konversation, fehlende Beachtung und erzieherische Einflussnahme und Förderbedarfe (z. B. durch Anregung zum Spielen). Sie kann sich auch im Mangel an Wärme in der Beziehung zum Kind und in der fehlenden Reaktion auf emotionale Signale des Kindes ausdrücken. Kindler unterscheidet hier nochmals differenzierter in emotionale und in kognitive Vernachlässigung.

Aktive Vernachlässigung wird als bewusste Vernachlässigung durch Essensentzug als Sanktion und Verweigerung von medizinischer Versorgung beschrieben, wohingegen die passive Vernachlässigung auch als unbewusste Vernachlässigung durch unzureichendes Wissen und mangelnde Aufmerksamkeit, zum Beispiel altersunangemessenes Alleinlassen über einen langen Zeitraum, definiert ist. Unkenntnis über den Aufenthaltsort des Kindes oder des_der Jugendlichen kann als aktive oder passive Vernachlässigung auftreten.

\section{Physische Gewalt}

Günther Deegener spricht bei physischer Gewalt explizit von »Ohrfeigen; Schlagen mit Händen, Stöcken, Peitschen; [...] Verbrennen mit 
heißem Wasser oder Zigaretten; auf den Ofen setzen; Einklemmen in Türen oder Autofensterscheiben; Piksen mit Nadeln; ins kalte Badewasser setzen und untertauchen; eigenen Kot essen und Urin trinken lassen; Würgen; Vergiftungen « (Deegener, 2005, S. 37).

Nicht zu unterschätzen sind die psychischen Auswirkungen bei Kindern und Jugendlichen, die körperliche Gewalt nach sich ziehen. Dazu gehören Verängstigung und Einschüchterung. Jede physische Gewalt ist auch eine seelische. Körperlich misshandelte Kinder werden zudem häufig vernachlässigt.

Züchtigung als Mittel zur Bestrafung von Kindern und Jugendlichen, die sich nicht entsprechend der Erwartungen der Eltern verhalten, wird heute teilweise noch immer, wenn auch nicht mehr so stark wie in der Vergangenheit als vertretbare Handlung betrachtet. »So glaubten 1996 z. B. noch 35\% der Eltern, auf Grund der Rechtslage ihrem Kind den $>$ Po versohlen $<$ zu dürfen, während es heute $>$ nur $<$ noch $19 \%$ sind « (ebd., S. 42). Erst im Jahr 2000 wurde durch das » Gesetzes zur Ächtung der Gewalt in der Erziehung und zur Änderung des Kindesunterhaltsrechts « im $\$ 1631$ des Bürgerlichen Gesetzbuches eine Passage aufgenommen, die das Recht von Kindern auf gewaltfreie Erziehung formuliert: » Kinder haben ein Recht auf gewaltfreie Erziehung. Körperliche Bestrafung, seelische Verletzung und andere entwürdigende Maßnahmen sind unzulässig«(\$1631 Abs. 2).

\section{Psychische Gewalt}

Psychische Gewalt lässt sich nur schwer an einzelnen Handlungen festmachen. Darüber hinaus ist sie nicht leicht von unpädagogischem, rüdem, falschem, aber noch rechtmäßigem Erziehungsverhalten abzugrenzen. Im Unterschied zur Vernachlässigung ist sie vorsätzlich und aktiv. Sie geht über eine Vernachlässigung hinaus und schädigt aktiv die Psyche und Entwicklungsmöglichkeit des Kindes oder des_der Jugendlichen. Das können dauerhafte alltägliche Beschimpfungen sein, aber auch das Isolieren und Einsperren gehört dazu - bis hin zu massiven Bedrohungen, sogar Todesandrohungen (vgl. Deegener, 2005, S. 38).

Krieger und Kolleg_innen beschreiben psychische Gewalt als den »Kern aller Misshandlungsformen « (Krieger et al., 2007, S. 17). Da- 
mit meinen sie, dass physische Gewalt und sexueller Missbrauch immer auch mit einer psychischen Gewalt einhergehen. Vergleichsweise häufig tritt psychische Gewalt aber auch als eine für sich alleine stehende Misshandlungsform auf (vgl. ebd., S. 14).

Im Handbuch Kindeswoblgefährdung nach \$1666 BGB und Allgemeiner Sozialer Dienst sind fünf Unterformen von psychischer Gewalt zu finden, die Kindler nach einer noch differenzierteren Auflistung von James Garbarino übernommen hat. Diese sind wie folgt beschrieben:

$>$ ständiges Herabsetzen, Beschämen

$>$ Zwang zu strafbarem oder selbstzerstörerischen Handeln

$>$ Verweigerung emotionaler Responsivität (Signale und Bedürfnisse des Kindes nach emotionaler Zuwendung werden anhaltend und in ausgeprägter Form übersehen und nicht beantwortet)

$>$ Isolation von altersentsprechenden sozialen Kontakten

$>$ ständiges Drohen bis hin zu Todesdrohungen (vgl. Kindler et al., 2006, S. 45)

Sowohl Kindler als auch die »American Professional Society on Abuse of Children « (APSAC) fassen unter den Begriff der psychischen Gewalt auch besondere Situationen wie:

$>$ Kinder werden Zeugen von elterlicher Gewalt untereinander (indirekte Form von psychischer Gewalt)

$>$ gezielte Entfremdung des Kindes vom getrenntlebenden Elternteil (vgl. Deegener, 2005, S. 111)

Sigrid Tschörpe-Scheffler beschreibt in ihrem Buch Fünf Säulen der Erziehung auch übermäßiges, altersunangemessenes »Behüten « des Kindes als eine Form der psychischen Gewalt. Deegener vertritt diese Meinung ebenfalls. Besonders beim Fehlen eines Elternteils muss hier die sehr frühe und dauerhafte Rollenzuschreibung eines Erwachsenen erwähnt werden.

Die Grenze zwischen psychischer Gewalt und einem noch tolerierbaren Erziehungsverhalten ist schwierig und beinhaltet eine Grauzone, die es genau zu durchschauen gilt. Die Gefährdung kann durch die Risikoabschätzung und daraus folgende Hilfsangebote abgewendet werden. 


\section{Sexueller Missbrauch}

Im Alltag und in der wissenschaftlichen Literatur existieren eine Vielzahl von Begriffen für diesen einen Tatbestand. Im Bereich der Kindeswohlgefährdung und in Abgrenzung zu sexualisierter Gewalt im Jugendverband und unter Jugendlichen soll für Formen von Kindeswohlgefährdung im familiären und sozialen Nahraum von Kindern und Jugendlichen der Begriff »sexueller Missbrauch « verwendet werden, da er so auch im Gesetz verwendet wird.

Kinder, die Opfer von sexuellem Missbrauch werden, sind häufig Drohungen, Gewalt- und Strafandrohungen ausgesetzt. Der psychische Druck ist meist enorm, dem die Opfer aufgrund der oft emotionalen Verbundenheit mit dem Täter oder der Täterin ausgesetzt sind. Sie trauen sich nicht, Hilfe zu holen und geben sich oft selbst die Schuld. Andreas Jud beschreibt zwei Aspekte bei Missbrauch durch Bezugspersonen: Zum einen sind die Betroffenen durch die Handlung einer Vertrauensperson mit der Verletzung persönlichster Erfahrungen konfrontiert, zum anderen mit einem Vertrauensbruch. Das zieht häufig Ambivalenzkonflikte nach sich (vgl. Fegert et al., 2015, S. 42). Wie mit Eltern bei innerfamilialem Missbrauch umgegangen wird, beschreibt Dirk Bange als kontrovers diskutiert. So sagt er, dass sich die Einbeziehung der Familien bei innerfamilialem Missbrauch in vielen Fällen wesentlich schwieriger gestaltet als bei einem Verdacht auf außerfamilialen sexuellen Missbrauch. In Familien passiert es häufig, dass sexueller Missbrauch übersehen, geleugnet oder totgeschwiegen wird. Bei der Risikoeinschätzung ist dies zu beachten und hier nochmals auf $\$ 8$ a Abs. 4 SGB VIII hinzuweisen: $\gg$ Dabei sind die Erziehungsberechtigten sowie das Kind oder der Jugendliche einzubeziehen, soweit hierdurch der wirksame Schutz des Kindes oder des Jugendlichen nicht in Frage gestellt wird. « So gilt in einem solchen Fall, die Gespräche mit dem nicht missbrauchendem Elternteil sorgfältig vorzubereiten. In der Regel sind Verantwortliche in der verbandlichen Jugendarbeit für solche Gespräche nicht ausgebildet und vorbereitet, daher ist es bei einem Verdacht auf sexuellen Missbrauch von Anfang an wichtig, eine Unterstützung durch spezielle Fachpersonen zu suchen und gegebenenfalls das Jugendamt einzuschalten. Besonders gilt das auch bei der Beteiligung der Be- 
schuldigten. Hier ist das Risiko besonders hoch, dass der Täter oder die Täterin »das Kind unter Druck « setzt und »den Kontakt zu den Hilfeeinrichtungen zu unterbinden « versucht (vgl. Fegert et al., 2015, S. 208f.).

\section{Erwachsenenkonflikte um das Kind}

Trennen sich Eltern, hat das immer auch Folgen für das Kind. Wenn die Bezugspersonen in dieser Situation durch Unterlassung oder aktives Handeln das Kind in diesen Konflikt involvieren, kann das Folgen für das psychische Wohl des Kindes haben.

Weitere Situationen sind hochstrittige »Familienkonflikte, Umgangskonflikte und Herausgabekonflikte zwischen Herkunftseltern und Pflegeeltern oder anderen Unterbringungseinrichtungen « (Kunkel, 2006, S. 22). Dabei sind Eltern oftmals durch ihre emotionale Betroffenheit nicht mehr in der Lage, ihre partnerschaftlichen Probleme getrennt von ihrer Verantwortung als Eltern zu sehen und zum Wohle der Bedürfnisse ihrer Kinder zu agieren. Mit diesem Verhalten zwingen Eltern ihr Kind in einen emotional sehr starken Konflikt. Wenn Kinder unvorbereitet in den Streit ihrer Eltern hineingezogen werden und als »Prellbock « für die Eltern herhalten müssen, werden sie in einen Loyalitätskonflikt gezwungen, der eine weitere Krisensituation für sie bedeutet.

Elternkonflikte um das Kind stellen für sich betrachtet in der Regel keine unmittelbare Gefahr für das Kindeswohl dar. Gleichwohl kann es in Kombination mit anderen Faktoren eine verstärkende Wirkung haben.

\section{Autonomiekonflikte junger Menschen}

Gerade im Bereich der Jugendarbeit kann diese Form relevant sein. Auf dem Weg zum Erwachsenwerden ist die Entwicklung der Autonomie wesentlich. Eine altersgerechte Verselbstständigung des_der Jugendlichen ist für die psychosoziale Entwicklung elementar. Diese üblichen Autonomiekonflikte stellen dann eine Gefahr für das Wohl des_der Jugendlichen dar, wenn Bezugspersonen diese Ablösung missachten oder verhindern. 


\section{Signale von Kindeswohlgefährdung}

Grundsätzlich gilt, dass es keine Signale gibt, die eindeutig auf das Vorliegen von Kindeswohlgefährdung hinweisen. Bestimmte Merkmale machen ein genaueres Hinschauen allerdings notwendig. Vor allem, wenn sie in Kombination mit anderen Merkmalen auftreten. Ein Indiz ist sicher das eigene »Bauchgefühl «. Eine plötzliche, vielleicht auch aggressive, Verhaltensänderung eines Kindes in der Kindergruppe, ein Mädchen, das nicht auf die Ausflüge mit darf, ein Kind, das nicht mitspielen darf, weil es unangenehm riecht, kann ein Hinweis sein, kann aber auch trügen, denn einige Verhaltens- und körperliche Veränderungen gehören zu normalen Entwicklungen im Kindesund Jugendalter oder auch zu einem jugendlichen Modestil. Von einer Kindeswohlgefährdung darf also nicht zwangsweise ausgegangen werden. Andererseits kann sie auch nicht grundsätzlich ausgeschlossen werden.

Signale sind in unterschiedlichen Bereichen zu finden: sei es in der kognitiven Entwicklung, dem psychischen oder sozialen Verhalten des Kindes oder des_der Jugendlichen, der körperlichen Versorgung, in der Beziehung und dem Verhalten zu wichtigen Bezugspersonen oder in der Wohnsituation.

Vorhandene Auflistungen von Signalen, wie sie auch im Anhang (A4) zu finden ist, darf nicht als eine Liste verstanden werden, an deren Ende die Antwort »ja « oder »nein « gegeben wird. Sie ist eher so zu verstehen, dass die aufgelisteten Signale dann eine Aufmerksamkeit erfordern, wenn mehrere dieser Hinweise beobachtet werden. Diese Zusammenstellungen, wie sie zum Beispiel auch die Ankerbeispiele für den Kinderschutzbogen beinhalten, dienen den Fachkräften bei der Einzelfalleinschätzung als Orientierung und erleichtern darüber hinaus die Kommunikation mit Eltern und in der kollegialen Beratung.

Lutz Goldberg fasst die benötigte Haltung von Fachkräften in der Arbeit mit Kindern und Jugendlichen als aufmerksame, jedoch gelassene Wahrnehmung von Auffälligkeiten im Verhalten der ihnen anvertrauten Kinder und Jugendlichen: »Sie sollten voreilige Rückschlüsse von unspezifischen Symptomen auf sexuellen Missbrauch unterlassen, allerdings offen für spontane Mitteilungen von Kindern und Jugendli- 
chen über sexuelle Übergriffe bleiben und darauf interessiert und mit Hilfeangeboten reagieren « (Fegert et al., 2015, S. 151).

\section{Gewichtige Anhaltspunkte}

Gewichtige Anhaltspunkte » sind das auslösende Moment für die Wahrnehmung des Schutzauftrages « (Wiesner, 2015, §8a Rn. 14). Darunter ist zu verstehen, dass konkrete Hinweise oder ernstzunehmende Vermutungen über eine Gefährdung vorliegen müssen.

Diese gewichtigen Anhaltspunkte müssen Fachkräfte durch eigene Wahrnehmung oder unmittelbar von Kindern, Eltern oder Dritten erhalten. Gewichtige Anhaltspunkte sehen je nach Arbeitsfeld spezifisch aus, auch sind altersspezifische Differenzierungen zu berücksichtigen. So sind »Lebensumstände bzw. Tun oder Unterlassen der Eltern mit den Bedürfnissen eines konkreten Kindes in Beziehung zu setzen « (Kindler et al., 2006, S. 35). Je nach Alter können Handlungen der Eltern, zum Beispiel Schütteln, erhebliche Gefahren sein.

Zur rechtzeitigen Einschätzung von Kindeswohlgefährdung verwenden Fachkräfte Indikatorenlisten, die auf eine im $\$ 1666$ BGB beschriebene Gefährdungslage hinweisen. Als ein Beispiel ist hier der Orientierungskatalog mit Ankerbeispielen für den Stuttgarter Kinderschutzbogen zu erwähnen. Die Ankerbeispiele sind aus dem Wissen und der Erfahrung der Sozialen Dienste der Jugendämter Stuttgart und Düsseldorf entstanden. Sie wurden mit der Methode der $\gg$ Kommunikativen Validierung « ${ }^{5}$ entwickelt und dienen der Orientierung bei der Einschätzung. Das Besondere dabei ist, dass die Ankerbeispiele in einzelne Altersmodule unterteilt sind (0-3, 3-6, 6-14 und 14-18 Jahre). Eine ideale Liste kann es jedoch nie geben, da die Einschätzung ein hochkomplexer Vorgang ist und nur im Gesamten zu betrachten sinnvoll und hilfreich ist. Dabei spielen neben Altersdifferenzierungen auch Differenzierungen nach Geschlecht und im Jugendalter sogar nach jugendkulturellen Szenen eine wichtige Rolle.

5 Im Dialog tauschen sich Forscher und Beforschte über die erarbeiteten Ergebnisse aus, daraus entstehen weitere wichtige Erkenntnisse, die wiederum in die Ergebnisse eingearbeitet werden. 


\section{Risikoeinschätzung}

Nach Kindler ist die Definition einer Risikoeinschätzung im Zusammenhang mit Kindeswohlgefährdung ein $\gg$ Prozess der Informationssammlung und der darauf aufbauenden fallbezogenen Überlegungen zur Wahrscheinlichkeit des zukünftigen Auftretens einer oder mehrerer Formen von Kindeswohlgefährdung « (Deegener, 2005, S. 53). Es liegt erst dann eine Kindeswohlgefährdung vor, wenn die Wahrscheinlichkeit hoch ist, dass eine geistige, seelische oder körperliche Beeinträchtigung vorhanden ist und nicht, wenn subjektiv das Kindeswohl nicht ausreichend gefördert wird.

Friederike Alle bezeichnet die Abschätzung als »eine äußerst komplexe und diffizile Aufgabe $\ll$ (Alle, 2010, S. 53). Durch diese multidimensionale Betrachtungsweise ist das Einbeziehen von mehreren Fachkräften und auch einer insoweit erfahrenen Fachkraft bei der Einschätzung notwendig. Eine Auseinandersetzung mit dem Thema und die damit verbundene Erlangung von Fachkenntnissen ist unabdingbar, um eine möglichst große Sicherheit zu erlangen, denn eine »Intervention im Zusammenhang mit einer Kindeswohlgefährdung kann für das Kind und auch die Familie sehr einschneidend [...] und mit langfristigen Folgen [...] verbunden sein « (ebd.).

Es gibt kein gesichertes System von Indikatoren für eine Gefährdung, bestimmte Anhaltspunkte können jedoch auf ein erhöhtes Risiko hinweisen. Es ist immer eine subjektive Sichtweise, ob man aus der Kumulation verschiedener Elemente möglicherweise Hinweise interpretiert, dass eine Risikoschwelle überschritten ist.

Nach Heinz Kindler und Wulfhild Reich hat der Begriff Abschätzung mindestens drei Aspekte (vgl. Kindler \& Reich 2006, S. 63):

1. Sammlung relevanter Informationen

2. Bewertung dieser Methoden

3. nachvollziehbare Dokumentation der Ergebnisse

Der Stuttgarter Kinderschutzbogen mit den Orientierungskatalogen für verschiedene Lebensalter kann hier ein hilfreiches Instrument sein. Im Rahmen des Stuttgarter Kinderschutzprojekts wurde er in einem Zeitraum von 18 Monaten zwischen Oktober 2000 und März 2002 entwickelt und erprobt. Das Deutsche Jugendinstitut (DJI) - in Per- 
son von Dr. Heinz Kindler - begleitete dieses Projekt wissenschaftlich. In Kooperation zwischen den Jugendämtern Stuttgart und Düsseldorf wurde dieser Bogen zum inzwischen wegweisenden Diagnoseinstrument zur Gefährdungseinschätzung weiterentwickelt. Die Grundlage für die darin aufgeführten Ankerbeispiele sind Längsschnittstudien zur Entstehung von Misshandlung, die auf ihre wissenschaftlich nachgewiesene Aussagekraft überprüft wurden.

Thomas Trenczek fasst das in der Kinderschutzpraxis bewährte Vorgehen in vier Fragen zusammen:

»1. Gewährleistungdes Kindeswohls: Inwieweit ist das Wohl des Kindes oder Jugendlichen durch die Eltern/Sorgeberechtigten gewährleistet oder ist dies nur zum Teil oder überhaupt nicht der Fall?

2. Problemakzeptanz: Sehen die Eltern/Sorgeberechtigten und die Kinder oder Jugendlichen selbst ein Problem oder ist dies weniger oder gar nicht der Fall?

3. Problemkongruenz: Stimmen die Eltern/Sorgeberechtigten und die beteiligten Fachkräfte in der Problemkonstruktion überein oder ist dies weniger oder gar nicht der Fall?

4. Hilfeakzeptanz: Sind die betroffenen Eltern/Sorgeberechtigten und Kinder oder Jugendlichen bereit, die ihnen gemachten Hilfeangebote anzunehmen und zu nutzen oder ist dies nur zum Teil oder gar nicht der Fall?«(Trenczek, 2008, S. 50).

Werden diese Fragen im Prozess der Risikoeinschätzung dahin gehend beantwortet, dass Eltern das Wohl des Kindes nicht gewährleisten können, die Probleme nicht als solche gesehen werden, die Sichtweise von Fachkräften und Eltern bezüglich des Problems kongruent sind und Eltern nicht bereit sind die Hilfsangebote zu nutzen, ist die Risikoeinschätzung dahin gehend zu interpretieren, dass das Wohl des Kindes gefährdet ist.

\section{Risikofaktoren für die Gefährdung des Kindeswohls}

In der Literatur wird seit einigen Jahr(zehnt)en darüber diskutiert, » welche Faktoren das Risiko für Mädchen und Jungen erhöhen, dass 
sie Opfer sexualisierter Gewalt werden « (Fegert et al., 2015, S. 104). So ist es in der Bewertung der Faktoren ausschlaggebend, wie hoch die statistische Wahrscheinlichkeit ist, dass bei vorliegenden Risiken auch eine Gefährdung vorliegt. Die Anzahl der Studien, die sich mit den Risikofaktoren für die Gefährdung des Kindeswohls auseinandersetzen ist in den letzten 30 Jahren beachtlich gewachsen (vgl. Kindler \& Lillig, 2005, S. 10). Auch wenn einzelne Faktoren für sich genommen kein Risiko darstellen und als Hinweise für eine spätere Entwicklungsstörung interpretierbar sind, können sie in Kombination durchaus eine Gefährdung begünstigen.

So werden im Allgemeinen folgende Risikofaktoren für eine Gefährdung der positiven und gesunden Entwicklung von Kindern genannt (vgl. Bender \& Lösel, 2005, S. $317 \mathrm{ff}$.; Kindler et al., 2006, S. 117ff.; Fegert et al., 2015, S. 104ff.):

$>$ niedriger sozioökonomischer Status

$>$ beengte Wohnverhältnisse großer Familien

$>$ geringer Altersabstand der Kinder von weniger als 18 Monaten

$>$ soziale Isolation der Familie durch wenige Sozialkontakte in der Nachbarschaft oder innerhalb der Familie

$>$ Belastungen der Eltern (psychische »Störungen «, schlechte Schulbildung, schwere Erkrankung, Alkohol-/Drogenabhängigkeit, starke berufliche Anspannung, eigene Gewalterfahrungen in der Kindheit)

$>$ Trennung/Verluste von Elternteilen

$>$ Konflikte in der Familie (Ehekonflikte, Erziehungsprobleme, Kriminalität)

Einige dieser Faktoren können auch unter der Überschrift »Krise « zusammengefasst werden: Krisen, in denen sich Familien oder einzelne Personen innerhalb von Familien befinden. Als Ursachen sind aber auch die eigene Hilflosigkeit und Überforderung der Bezugspersonen zu benennen.

Kunkel beleuchtet in seiner Ausführung der Entstehungsbedingungen auch die gesellschaftlichen Entwicklungen und Phänomene, »die das Auftreten von Misshandlungen begünstigen, wie ein generell hohes $\mathrm{Maß}$ an Gewaltbereitschaft, [...] [die] befürwortende oder zumindest ambivalente Haltung gegenüber Körperstrafen als Erziehungsmittel und eine mangelnde Akzeptanz von Kindern und ihrer Rechte « (Kun- 
kel, 2006, S. 23). Auch Bange beschreibt Risiken aus dem Einfluss des gesellschaftlichen und kulturellen Kontextes. Er zählt hier zu den Risiken die Einstellung zur kindlichen Sexualität, den (leichten) Zugang zu Kinderpornografie, wenige Rechte von Kindern und patriarchale Strukturen. Zudem können sich alkoholisierte Täter_innen aus ihrer Verantwortung ziehen, wenn es innerhalb der Gesellschaft eine hohe Toleranz gegenüber Taten unter Alkoholeinfluss gibt oder sexualisierte Gewalt strafrechtlich nur sehr gering verfolgt wird (vgl. Fegert et al., 2015, S. 106).

Auch Risiken auf der Ebene des Kindes, die eine Gefährdung des Kindeswohls begünstigen, sind als Faktoren zu berücksichtigen. Dazu gehören unter anderem:

$>$ Frühgeburten und damit verbundene Erschwernis des Beziehungsaufbaus zur Bezugsperson

$>$ Kinder mit Behinderung und gleichzeitig geringeren Möglichkeiten, sich zur Wehr zu setzen (vgl. ebd., S. 105)

$>\quad \gg$ Schreikinder $\ll$

$>$ Kinder mit Verhaltensauffälligkeiten wie Hyperaktivität

$>$ Mädchen

Tschörpe-Scheffler weist mit ihrem Modell der »Fünf Säulen der Erziehung « auf die entwicklungsfördernde und entwicklungshemmende Unterstützung der Eltern durch das Erziehungsverhalten hin. Förderlich und damit wenig risikobehaftet ist ein eindeutiges $\gg j a \ll$ zum Kind, zur Verantwortung und Zuständigkeit und zu Übernahme der Mutter- und Vaterrolle. Dieses zeigt sich in den fünf Säulen Liebe, Achtung, Kooperation, Struktur und Förderung (vgl. Tschörpe-Scheffler, 2009, S. 305f.). Das Modell ist ein idealtypisches und stellt mit dem Gegenüber der entwicklungshemmenden Erziehung eine Vorlage zur Reflexion des eigenen Verhaltens von Eltern und Pädagog_innen dar. So gilt es, mithilfe dieser Strukturelemente, »entwicklungsförderndes Verhalten $\mathrm{zu}>$ maximieren < und entwicklungshemmendes zu $>$ reduzieren < (ebd., S. 307). Als entwicklungshemmende Unterstützung der Eltern beschreibt Tschörpe-Scheffler emotionale Kälte und dessen anderes Extrem, die Überfürsorge, die Missachtung, Chaos, die mangelnde Förderung und Überforderung des Kindes (vgl. ebd., S. 307ff.). 
Dirk Bange nennt als Schutzfaktoren auf der Ebene des Kindes ein hohes Selbstbewusstsein und gute schulische oder sportliche Leistungen, die sich wechselseitig verstärken und damit zusammenhängen. Schutzfaktoren innerhalb der Familie sei eine gute Bindung innerhalb des Familiensystems zu einem Elternteil oder auch zu einem Geschwisterkind (Fegert et al., 2015, S. 105f.).

\section{Datenschutz}

Durch die Neuregelung des SGB VIII haben sich Änderungen ergeben, die den Datenschutz einschränken und die Datenweitergabe im Zusammenhang mit dem Schutzauftrag bei Kindeswohlgefährdung in gewisser Weise erleichtern.

Grundsätzlich hat jeder das Recht, selbst über die Preisgabe und Verwendung seiner persönlichen Daten zu bestimmen (vgl. Art. 1 in Verbindung mit Art. 2 GG), daher ist eine Datenübermittlung grundsätzlich zulässig, wenn die Einwilligung des Betroffenen vorliegt. Diese Einwilligung muss schriftlich erfolgen. In der Kinderschutzpraxis ist es daher wichtig, den Eltern, dem Kind oder einem anderen Übermittler der Sozialdaten transparente Information über die genaue Weitergabe und den Folgen daraus für eine weitere Zusammenarbeit zu geben (vgl. Ministerium für Bildung, 2005, S. 9). Von einer Zustimmung kann nicht immer ausgegangen werden. Zu beachten ist, dass eine Weitergabe der Informationen »ohne entsprechendes Einverständnis von Eltern und deren Kindern [...] eine massive Belastung der Vertrauensbeziehung « (Meysen, 2007, S. 32) darstellt.

Wenn die Rechte von Kindern und Jugendlichen verletzt werden, ihr Wohl gefährdet ist, tritt der Datenschutz in den Hintergrund. So ist ein Rechtfertigungsgrund zur Weitergabe der Daten immer dann gegeben, wenn gewichtige Anhaltspunkte für eine Kindeswohlgefährdung vorliegen. Diese rechtfertigenden Notsituationen sind in $\$ 34$ StGB beschrieben und erlauben eine Weitergabe der Informationen dann, wenn nur dadurch die Gefahr vom Kind abgewendet werden kann.

Im Zusammenhang mit der Erfüllung des Schutzauftrags bei Kindeswohlgefährdung sind die \$\$61-65 SGB VIII als Grundlage zu sehen. Nach $\$ 61$ Abs. 3 SGB VIII gelten die Bestimmungen zum Da- 
tenschutz bei freien Trägern der Jugendhilfe durch Vereinbarungen entsprechend.

Die Datenerhebung regelt $\$ 62$ SGB VIII. Demnach dürfen alle Daten erhoben werden, die für die Erfüllung des Schutzauftrages nach $₫ 8 \mathrm{a}$ SGB VIII notwendig sind (vgl. $\$ 62$ Abs. 1 SGB VIII). Diese Daten müssen allerdings nach Absatz 2 bei der betroffenen Person selbst erhoben werden und diese muss über den Zweck informiert werden. Abs. 3 Nr. 2d erlaubt die oft erforderliche Erhebung der Daten von Dritten bei einer Gefährdungsabschätzung.

Vom Gesetzgeber werden anvertraute und nicht anvertraute Sozialdaten unterschieden. Sozialdaten umfassen alle personenbezogenen Angaben, die zu einer Identifizierung und Charakterisierung einer Person beitragen und die im Zusammenhang mit Jugendhilfeaufgaben erhoben oder verwendet werden (vgl. $\$ 67$ Abs. 1 SGB X). Anvertraute Daten sind besonders geschützt und wurden von der betreffenden Person erzählt. Nicht anvertraute Sozialdaten wurden durch Beobachtung erhoben.

Nicht anvertraute Sozialdaten dürfen zu dem Zweck, zu dem die Daten erhoben wurden, weitergegeben werden (vgl. \$64 Abs. 1 SGB VIII).

Die Übermittlung von anvertrauten Daten ist nur zulässig, wenn die betroffene Person einwilligt ( $\$ 65$ Abs. 1 Nr. 1 SGB VIII) bzw. wenn es gesetzlich erlaubt ist. Diese gesetzliche Erlaubnis der Datenübermittlung im Zusammenhang mit der Erfüllung des Schutzauftrages und drohender Misshandlungsgefahr leitet sich aus $\$ 65$ Abs. 1 Nr. 2-5 SGB VIII ab. Für Freie Träger der Jugendhilfe gibt es hier die Möglichkeit, bei einer Gefährdung von Kindern bzw. Jugendlichen andere Fachkräfte zur Fachberatung gemäß \$8a hinzuzuziehen. In diesem Fall kann die Übermittlung der anvertrauten Daten auch ohne Zustimmung der Betroffenen an die Fachberatung erfolgen.

Der Gesetzgeber hat mit der Regelung den Fachkräften der freien Jugendhilfe eine Verantwortung übergeben, die darin besteht, dass diese Fachkräfte die Hemmschwelle gegenüber dem Jugendamt abbauen und auf die Annahme der Hilfen vonseiten der Erziehungsberechtigten hinwirken (vgl. Meysen, 2007, S. 32). Nach $\$ 64$ Abs. 2a SGB VIII ist die Übermittlung der Sozialdaten an eine Fachkraft, die der Einrichtung nicht angehört in anonymisierter oder pseudonymisierter Form möglich. 
Unabhängig davon, ob ein_e Mitarbeiter_in bei einem Träger der öffentlichen oder der privaten Jugendhilfe tätig ist, muss jede_r einzelne Mitarbeiter_in die strafrechtliche Schweigepflicht (besser bekannt als ärztliche Schweigepflicht) beachten, wenn er einer der in $\$ 203$ Abs. 1 StGB genannten Berufsgruppen angehört (Jugendberater_innen, staatlich anerkannte Sozialarbeiter_innen oder staatlich anerkannte Sozialpädagog_innen).

Der oftmals wichtige Austausch im Netzwerk des Stadtteils und im Gemeinwesen ist laut Gesetz nicht vorgesehen und auch nicht erlaubt. Für die Risikoeinschätzung wäre das in manchen Fällen hilfreich. Das beschreibt auch Thomas Meysen mit den Worten: »Gelebte Kooperation bedarf der Kommunikation. Doch so einfach lässt sich das zwischen Professionellen in helfenden Berufen nicht bewerkstelligen « (ebd., S. 30). Durch das Recht wird das Vertrauensverhältnis zwischen Klient und Helfer geschützt, doch oft stellt sich der Erfolg der Hilfen durch die interinstitutionelle Kommunikation ein. Es gibt Netzwerke in Stadtteilen, deren Einrichtungen sich bei der Anmeldung der Kinder von dieser Schweigepflicht entbinden lassen. Sie argumentieren den Eltern gegenüber, dass dies zum Schutz des Kindes sei. Betroffene Familien »haben in aller Regel Angst und Scham vor einer Offenbarung ihrer Problematik, vor einem Verlust elterlicher Autonomie, davor, dass ihnen ihre Kinder weggenommen werden « (ebd., S. 31). Ob daher dieser Weg ein geeignetes Mittel ist oder diejenigen abschreckt, bei denen ein Risiko besteht, muss an anderer Stelle diskutiert werden.

Im $\$ 72 \mathrm{a}$ ist noch eine weitere Rede vom Datenschutz. Hier geht es um die Dokumentation der Einsichtnahme in das erweiterte polizeiliche Führungszeugnis. Hier wird genau beschrieben, wie die Dokumentation rechtlich sicher zu erfolgen hat:

»Träger der öffentlichen und freien Jugendhilfe dürfen [...] das Datum des Führungszeugnisses und die Information erheben, ob die das Führungszeugnis betreffende Person wegen einer Straftat nach Absatz 1 Satz 1 rechtskräftig verurteilt worden ist. Die Träger der öffentlichen und freien Jugendhilfe dürfen diese erhobenen Daten nur speichern, verändern und nutzen, soweit dies zum Ausschluss der Personen von der Tätigkeit, die Anlass zu der Einsichtnahme in das Führungszeugnis gewesen ist, erforderlich ist « (vgl. \$72a Abs. 5 SGB VIII). 
So sagt auch Wiesner in seinem Kommentar zum $\$ 72 \mathrm{a}$ SGB VIII, dass bei vorliegendem Tätigkeitsausschluss der Name der Person und die Tatsache dessen, dass ein Tätigkeitsausschluss vorliegt, sofern die Tätigkeit schon aufgenommen wurde, dokumentiert werden darf. Die Löschung der Daten muss spätestens drei Monate nach Beendigung der Tätigkeit erfolgen. Sollte die Tätigkeit noch nicht aufgenommen worden sein, so muss die Information sofort gelöscht werden. Bei Personen ohne verwertbaren Eintrag in das erweiterte Führungszeugnis darf nach den Bestimmungen des $\$ 72$ a nur der Name und das Datum der nächsten Einsichtnahme dokumentiert werden (vgl. Wiesner, 2015, Rn. 48).

Vorlagen, zum Beispiel des KVJS, folgen diesem Kommentar nicht ganz, wenn sie die Möglichkeit der Kennzeichnung von Tätigkeitsausschluss durch »Ja «- und »Nein «-Kästchen im Dokumentationsbogen aufnehmen. Ein Vorschlag zur Dokumentation findet sich im Anhang (A5).

Die Daten sind vor dem Zugriff Unbefugter zu schützen. Auch bei keinem Eintrag im erweiterten Führungszeugnis sind die Daten unverzüglich zu löschen, wenn im Anschluss an die Einsichtnahme keine Tätigkeit nach Abs. 3 Satz 2 oder Abs. 4 Satz 2 wahrgenommen wird. Andernfalls sind die Daten spätestens drei Monate nach der Beendigung einer solchen Tätigkeit zu löschen. 


\section{Die Bedeutung des Schutz- auftrags für die verbandliche Jugendarbeit}

Freiwilligkeit, Selbstorganisation und Partizipation sind die Schlagworte, welche die Prinzipien der verbandlichen Jugendarbeit beschreiben. Eine Beteiligungs- und Lebensweltorientierung sind die Qualitätsmerkmale von Jugendarbeit. Diese Strukturmerkmale sind bei der Entwicklung von Konzepten und der Wahrnehmung von Risikofaktoren für die verbandliche Jugendarbeit zu beachten. Im Kinder- und Jugendhilfegesetz hat der Gesetzgeber dieser besonderen Rolle von Jugendverbänden, -gruppen und Initiativen einen eigenen Paragrafen gewidmet. So beschreibt der $\$ 12$ SGB VIII diese Selbstorganisation, -verwaltung und Mitverantwortung. Die Interessen von jungen Menschen werden durch die Jugendverbände vertreten. Kinder- und Jugendverbandsarbeit ist durch soziale, politische und gesellschaftliche Wertvorstellungen geprägt und kann somit nie wertfrei sein. Der Kommunalverband für Jugend und Soziales Baden-Württemberg (KVJS) beschreibt das Wesen der verbandlichen Jugendarbeit als Förderung von jungen Menschen zu eigenverantwortlichen und gemeinschaftsfähigen Mitgliedern der Gesellschaft. Jugendarbeit richtet sich an alle. Die Beteiligung und Teilnahme an den Angeboten, Gruppen und Projekten ist freiwillig. In der Regel richten sich diese Angebote an die Kinder und Jugendlichen direkt, oft auch ohne festen Austausch und Kontakt mit den Eltern. Auch gesetzlich ist festgeschrieben, dass die Arbeit mit den Eltern kein Merkmal der Jugendarbeit ist (vgl. $\$ \$ 11$ und 12 SGB VIII). Die Fachkräfte und ehrenamtlich Mitarbeitenden in der Jugendarbeit sind oft in engem Kontakt mit den Kindern und 
Jugendlichen und stellen Vertrauenspersonen dar (vgl. KVJS, 2009, S. 37).

Die Interessen der Kinder und Jugendlichen stehen in der verbandlichen Jugendarbeit im Vordergrund. Sie ist lebensweltlich und sozialräumlich orientiert. Erste Erfahrungen in der Mitwirkung, -bestimmung und -verantwortung machen Kinder und Jugendliche in der Gruppenarbeit. In Seminaren zur Mitarbeiter_innenbildung, den Jugendleiterschulungen (JuLeiCa-Schulungen), steigen sie in den Prozess der Meinungsbildung und Entscheidungsfindung im Verband ein. Jugendliche und junge Erwachsene können sich in der verbandlichen Jugendarbeit frei entwickeln und ausprobieren, bis hin zur Übernahme von Leitungsfunktionen. Häufig gibt es einen fließenden Übergang zwischen Teilnehmen und Leiten. Jugendverbandsarbeit unterstützt Kinder und Jugendliche in der Wahrnehmung eigener Bedürfnisse und der Formulierung dieser in öffentlichen und politischen Gremien. Durch nicht formelle Bildungsprozesse hat die verbandliche Kinder- und Jugendarbeit einen besonderen Stellenwert in der Entwicklung von Kindern und Jugendlichen. Die sogenannten »Soft Skills « wie Verantwortungsgefühl, Sozialkompetenz, Teamfähigkeit und Kritikfähigkeit sind nicht in der Weise über einen formellen Bildungsprozess in der Schule zu erwerben, wie es in den nicht formellen Bildungsprozessen der verbandlichen Kinder- und Jugendarbeit der Fall ist.

Ein Wesensmerkmal der verbandlichen Jungendarbeit ist das ehrenamtliche Engagement. Jugendliche und junge Erwachsene engagieren sich unentgeltlich, freiwillig und selbstbestimmt; dennoch ist das Engagement von Verbindlichkeit und Verantwortung geprägt. Auch wenn das Engagement zeitlich befristet ist.

Im Hintergrundpapier des Deutschen Bundesjugendrings zum Schutzauftrag wird darauf hingewiesen, dass die Umsetzung des Schutzauftrages in der verbandlichen Jugendarbeit sowohl Chancen als auch Risiken birgt. Zum einen sind die Gruppenleiter überwiegend ehrenamtlich Mitarbeitende, die keine Experten für Kinderschutz sind. Zum anderen haben sie aber durch ihre Nähe zu den Kindern und Jugendlichen einen Vertrauensvorschuss gegenüber den Mitarbeitenden des öffentlichen Trägers der Jugendhilfe, sodass sie leichter kleine Signale, seien sie offen oder versteckt, als Hilferufe interpretieren können (vgl. DBJR, 2010, S. 1f.). 
Laut dem Deutschen Verein für öffentliche und private Fürsorge wäre die Kinder- und Jugendhilfe ohne ehrenamtliches Engagement nicht denkbar. Für ihn »ist es daher von großer Bedeutung, die Prävention in diesem Feld als Teil eines allgemein akzeptierten Selbstverständnisses und einer täglich gelebten Normalität herauszubilden, ohne dabei eine Atmosphäre von Verdächtigungen und Misstrauen zu schaffen « (Deutscher Verein, 2012, S. 2f.). Im weiteren Verlauf seiner Empfehlung weist er daraufhin, dass Gefährdungssituationen und Übergriffe nicht auf den Bereich der Kinder- und Jugendhilfe beschränkt sind, sondern überall dort, wo Kinder und Jugendliche in Angeboten zu finden sind. Daher ist seine Forderung, für alle Arbeitsbereiche (außerhalb der Jugendhilfe, im sportlichen, kulturellen oder schulischen Bereich u. a.) Präventionsangebote und Schutzkonzepte zu entwickeln (vgl. ebd., S. 6).

Die verbandliche Jugendarbeit ist aufgefordert, ehrenamtlich Engagierte für die Thematik des Schutzauftrages zu sensibilisieren und darin zu qualifizieren, wesentliche Anhaltspunkte für Misshandlung zu erkennen und professionell mit der Erkenntnis umzugehen. Weiter sind fachlich kompetente Ansprechpersonen zu benennen. Drei Jahre nach Einführung des Bundeskinderschutzgesetzes sind zahlreiche Jugendverbände sich dieser Verantwortung bewusst. Seit 2009 gibt es in den bundesweit einheitlichen Qualitätsstandards für die Schulungen im Rahmen der Jugendleitercard (JuLeiCa-Schulungen) das Thema Kindeswohlgefährdung als festen Bestandteil.

Vielfältig findet man in der Literatur Empfehlungen zu und Konsequenzen aus den $\$ \$ 8$ a und 72 a SGB VIII für die verbandliche Kinderund Jugendarbeit. Die Inhalte sind ähnlich und behandeln die gleichen Themen. Erwähnt sei hier die Empfehlung des Deutschen Bundesjugendrings (DBJR, 2006) und von Erich Jordan und Sigrid Bathke (Jordan \& Bathke, 2006, S. 97). Zusammengefasst kann man die dort aufgeführten Konsequenzen so formulieren:

$>$ Sensibilisierung und Qualifizierung aller Mitarbeitenden (ehren- und hauptamtlich Beschäftigter) und auch der Kinder und Jugendlichen

- verbindliche Aufnahme des Themenfeldes in die Ausbildung zum Jugendleiter und zur Jugendleiterin

$>$ thematische Bearbeitung des Themenfeldes in der Gruppenarbeit

$>$ fachlicher Austausch der hauptamtlich Beschäftigten 
$>$ Entwicklung von strukturellen Rahmenbedingungen, Transparenz im Verband, um Übergriffe zu verhindern bzw. schnellstmöglich aufdecken zu können

$>$ Entwicklung und Verankerung von allgemeingültigen und internen Verfahrensregelungen und Dokumentationen

$>$ genaueres Hinschauen der Fachkräfte und Hinzuziehen von erfahrenen Fachkräften bei Verdachtsmomenten auf Kindeswohlgefährdung

$>$ Selbstverpflichtungserklärungen für ehrenamtlich Mitarbeitende $>$ Benennen von Ansprechpartnern

Gunda Voigts hält darüber hinaus die Entwicklung von einer Handreichung für Ehrenamtliche und von speziellen Informationsangeboten für den Bereich der Ferienaktionen und -fahrten für notwendig (vgl. Voigts, 2005, S. 50).

Diese Empfehlungen finden sich auch im Abschlussbericht des Runden Tisches Sexueller Missbrauch, der von 2010 bis 2011 von der damals eingesetzten Unabhängigen Beauftragten für Kindesmissbrauch (UBSKM) Dr. Christiane Bergmann einberufen wurde. Ihr Nachfolger Johannes-Wilhelm Röhrig hat mit der Kampagne »Kein Raum für Missbrauch $\ll^{6}$ diese Empfehlungen weiterentwickelt und als Bestandteile von Schutzkonzepten beschrieben. Somit gehört zu der Auseinandersetzung mit dem Schutzauftrag Folgendes:

$>$ Leitbild, das heißt festgeschriebenes Bewusstsein über die Präventionsverantwortung innerhalb des Arbeitsfeldes

$>$ Verhaltenskodex, das heißt Verhaltensregeln innerhalb des Arbeitsfeldes (siehe Anhang A9)

$>$ Fortbildungen für mit Kindern- und Jugendlichen arbeitenden haupt- und ehrenamtlichen Personen

$>$ erweitertes Führungszeugnis und die Regelungen hierzu

$>$ Partizipation von Kindern und Jugendlichen als fester Bestandteil der Arbeit

$>$ Präventionsangebote für Kinder und Jugendliche

$>$ Informationsveranstaltungen über die Präventions- und Schutzkonzepte für alle Beteiligten, auch für die Eltern 
$>$ Beschwerdeverfahren mit Ansprechpersonen, welches im Falle einer Vermutung genutzt werden kann

$>$ Notfallplan für den Krisenfall und bei Verdacht (siehe Anhang A8)

$>$ Kooperation mit Fachberatungsstellen zur fachlichen Beratung und Unterstützung (vgl. Fegert \& Wolff, 2015, S. 429ff.)

Die verbandliche Jugendarbeit war in den letzten Jahren sehr aktiv in der Entwicklung und Umsetzung von präventiven Schutzmaßnahmen für Kinder und Jugendliche. So sind einige beachtenswerte Modelle entstanden. Auch wenn ehrenamtlich Tätige keine Fachkräfte im Sinne des $\$ 72$ SBGB VIII sind, so haben sie trotzdem durch das allgemeine Verständnis von Jugendverbandsarbeit bei einem Fall von Kindeswohlgefährdung oder bei Grenzüberschreitungen, Übergriffen oder gar sexualisierter Gewalt durch Betreuungspersonen im Verband zu handeln. Dieses Handeln sollte in Schulungen besprochen werden.

Eine große Stärke von verbandlicher Jugendarbeit ist die gemeinsame Entwicklung von Themen mit Kindern und Jugendlichen. Durch die Nähe zur Lebenswirklichkeit der Kinder und Jugendlichen ist hier gerade ein präventiver Ansatz möglich, der nicht über ein »vordefiniertes Curriculum « eingeschränkt wird (vgl. Deinet, 2006, S. 28). Ebenso unterstreicht das Hintergrundpapier des DBJR von 2010 diesen Gesichtspunkt:

»Die Jugendverbände leisten mit ihrem Angebot und ihrer Methodik einen zentralen Beitrag gegen Gewalt an Kindern. Sie stärken Kinder und Jugendliche in ihrer Persönlichkeit und unterstützen sie, ihre eigenen Grenzen zu erkennen und selbstbewusst zu artikulieren « (DBJR, 2010, S. 1).

So kann man die Bedeutung des Schutzauftrages in der verbandlichen Jugendarbeit vom Selbstverständnis ableiten, ein sicherer und geschützter Raum für Kinder und Jugendliche zu sein, der auch ohne eine Vereinbarung mit dem öffentlichen Träger gestaltet wird. Vor diesem Hintergrund kann die Konsequenz gesehen werden, dass die verbandliche Jugendarbeit ihre ehrenamtlich Mitarbeitenden für das Thema sensibilisieren und schulen soll. Durch diese gedankliche Vorbereitung 
auf konkrete Verdachtsmomente im Vorhinein können Abläufe standardisiert werden. Im konkreten Verdachtsmoment und dem dadurch ausgelösten Stress und der Unsicherheit kann eine solche Maßnahme hilfreich sein. Wichtig ist hierbei, die Engagierten nicht zu überfordern, sondern ihnen Kompetenzen zu vermitteln, die es ihnen ermöglicht, aktiv zu werden.

\section{Vereinbarungen mit dem Jugendamt}

Durch die Veränderungen im SGB VIII durch das Bundeskinderschutzgesetz haben sich auch die Voraussetzungen für die Vereinbarungen mit dem Jugendamt verändert. Hier ist eine Differenzierung der Vereinbarungen notwendig:

$>$ Vereinbarungen im Zusammenhang mit dem Tätigkeitsausschluss einschlägig vorbestrafter Personen (\$72a SGB VIII)

$>$ Vereinbarungen zum Schutzauftrag bei Kindeswohlgefährdung (\$8a SGB VIII)

\section{Vereinbarungen nach §72a SGB VIII}

Im Bereich der Vereinbarungen nach $\$ 72$ a SGB VIII ist die Einschränkung auf freie Träger von Diensten und Einrichtungen entfallen. Seit der Neuregelung beziehen sich die Vereinbarungen eindeutig auf alle freien Träger. Somit ist der Bereich des Tätigkeitsausschlusses einschlägig vorbestrafter Personen in der Arbeit mit Kindern und Jugendlichen durch Vereinbarung mit dem öffentlichen Träger auch ein Thema für die verbandliche Jugendarbeit.

Die im Vorfeld der Einführung des Bundeskinderschutzgesetzes geäußerte Kritik des bürokratischen Aufwandes vonseiten der Jugendverbände wurde innerhalb des Evaluationsberichtes der Bundesregierung als »gering « eingestuft und in den Forschungsergebnissen als nicht bestätigt erwiesen. Hier kritisiert der Deutsche Bundesjugendring das Vorgehen der Evaluation, denn » [b] elastbare Forschungsergebnisse liegen zu diesem Thema insbesondere deswegen nicht vor, weil [...] diese Thematik und hier u.a. die Arbeit der Jugendverbände, im Rahmen 
der Evaluation nicht tiefergehend untersucht wurde « (DBJR, 2016, S. 4).

Hier forderte der Deutsche Bundesjugendring in seiner ersten Stellungnahme 2015 eine Entbürokratisierung und im Hinblick auf den Datenschutz eine gute Lösung (DBJR, 2015). Dass hier das Wie und nicht das $O b$ infrage gestellt wird, zeigt den hohen Stellenwert des Kinderschutzes und des Verantwortungsbewusstseins in der verbandlichen Jugendarbeit.

Wie sich die Gesetzeslage in diesem Punkt ändert, kann zum jetzigen Zeitpunkt (August 2016) noch nicht gesagt werden, da mögliche Änderungen des SGB VIII bisher nicht öffentlich diskutiert werden.

\section{Vereinbarungen nach §8a SGB VIII}

Bei den Adressaten der Vereinbarungen gibt es weiterhin die Einschränkung auf Einrichtungen und Dienste der Jugendhilfe. Und somit betrifft die Regelung nur den Teil der verbandlichen Jugendarbeit, die Träger von Einrichtungen sind. Zu den Vereinbarungen bei Verdacht auf Kindeswohlgefährdung wurden vor allem vor dem Inkrafttreten des Bundeskinderschutzgesetzes viele Stellungnahmen und Einschätzungen veröffentlicht. So vertrat Gunda Voigts die Meinung, dass » wir Ehrenamtliche mit Vereinbarungen zur Frage des Kinderschutzes, zumindest in der Jugendarbeit « überfordern (Voigts, 2006, S. 177). Sie begründet ihre Haltung damit, dass in der verbandlichen Kinderund Jugendarbeit überwiegend ehrenamtliche Jugendliche eine Gruppe leiten. Dennoch sieht sie es für notwendig an, ehrenamtlich Mitarbeitende nicht vollständig aus der Verantwortung, die die Thematik des Kinderschutzes mit sich bringt, herauszuhalten. Auch sie hat darauf hingewiesen, dass Vereinbarungen mit Jugendverbänden nur dann abgeschlossen werden sollen, wenn diese Träger von Einrichtungen und Diensten sind, jedoch sollte »im Einzelfall überlegt werden, ob es sinnvoll ist, eine freiwillige Vereinbarung abzuschließen « (Voigts, 2005, S. 51), wenn der Jugendverband dies wünscht. Sie weist darauf hin, dass dies dann aber eine für beide Seiten gewinnbringende Kooperationsvereinbarung sein muss, die sich nicht auf den $\$ 8$ a stützen kann. Diese Vereinbarungen 
$>$ dürfen keine negativen Auswirkungen auf die finanzielle Gestaltung des Verbandes haben,

$>$ dürfen das staatliche Wächteramt nicht auf den Jugendverband übertragen,

$>$ müssen auch Serviceleistungen des Jugendamtes beinhalten und

$>$ sollten gemeinsam erarbeitetet Standards für Verfahrensabläufe festhalten (vgl. ebd., S. 51f.).

In Baden Württemberg hat sich eine Arbeitsgruppe unter Federführung des Kommunalverbands für Jugend und Soziales (KVJS) gebildet, die 2006 speziell für den Bereich der Jugendarbeit Regelungen bezüglich der Vereinbarung mit Jugendämtern erarbeitet hat. Diese Hinweise wurden nach dem Inkrafttreten des Bundeskinderschutzes im Februar 2014 angepasst und ergänzt. Die Veröffentlichung erfolgte über ein gemeinsames Rundschreiben mit Anlagen des Ministeriums für Arbeit und Soziales, des Ministeriums für Kultus, Jugend und Sport sowie des Kommunalverbandes für Jugend und Soziales. In diesen Hinweisen wird sehr deutlich dargestellt, dass beim Abschluss von Vereinbarungen zwischen Jugendämtern und Trägern von Einrichtungen und Diensten in den Arbeitsfeldern Jugendarbeit etc. arbeitsfeldspezifische Besonderheiten berücksichtigt werden müssen. Eine ganz wesentliche Aussage darin ist:

$\gg$ Es erscheint deshalb wenig sinnvoll, mit Jugendorganisationen und Jugendverbänden förmliche Vereinbarungen nach §8a SBG VIII abzuschließen, es sei denn, die Jugendorganisation betreibt eine Einrichtung mit hauptamtlichen Fachkräften, die mit Mitteln der öffentlichen Jugendhilfe bzw. von Gemeinden finanziell gefördert wird (z. B. Jugendzentrum). Bildungsstätten dieser Organisationen, in denen lediglich kurzfristige Veranstaltungen durchgeführt werden, können ebenfalls von Vereinbarungen ausgenommen werden $\ll(K V J S, 2014$, S. 8).

\section{Regelungen und Transparenz im Verband}

Beschäftigt man sich mit den Risikobereichen, so findet man in der Fachliteratur viele Hinweise auf das besonders hohe Risiko für Macht- 
missbrauch und Grenzverletzungen in Arbeitsfeldern, in denen zwischen Erwachsenen und Jugendlichen eine emotionale und dichte Beziehung besteht, sei sie ehrenamtlich oder beruflich (vgl. Fegert \& Wolff, 2015, S. 307). Bei der Aufdeckung von Fällen sexualisierter Gewalt in Internaten und Schulen der letzten Jahre wurde deutlich sichtbar, dass die Struktur der Einrichtung im Zusammenhang mit Gewalt eine entscheidende Rolle spielt.

Daher ist ein transparenter Umgang mit dem Thema von hoher Wichtigkeit. In der Literatur finden sich Hinweise auf den Zusammenhang von Organisationsstruktur und Schutz vor sexueller Gewalt.

In einem Fachbeitrag von Beate Steinbach wird dieser Zusammenhang erläutert. Nach deren Erkenntnis bietet eine nach außen abgeschottete Organisation mit großem Machtgefälle und autoritären Leitungsstrukturen große Möglichkeiten, sexuelle Gewalt zu vertuschen und zu verdecken. Sie erwähnt aber auch, dass sogenannte »Laissezfaire «-Organisationen dieses Risiko ebenso in sich tragen. Ein partnerschaftlicher Umgang und transparente Leitungsstrukturen sind immer wieder Thema der Auseinandersetzung in der verbandlichen Jugendarbeit. Die Umsetzung gelingt nur durch Mitwirkung aller Beteiligten und sollte immer wieder Thema auf allen Ebenen des Verbandes sein (Steinbach, 2015, S. 186f.).

Verbandliche Jugendarbeit kann sich im Bereich der Prävention stark für den Schutz von Kindern einsetzen. Prävention hat jedoch zwei Seiten: Zum einen geht es um die pädagogischen Methoden in der konkreten Arbeit mit den Kindern und Jugendlichen. Hier gibt es viele Arbeitshilfen und Auseinandersetzungen, die das Selbstverständnis der verbandlichen Jugendarbeit widerspiegeln.

Ein weiterer wichtiger Aspekt der Prävention sind aber auch die strukturellen Methoden. Dazu gehören die organisatorischen Strukturen des Verbandes. Wesentlich zu einer strukturellen Prävention gehört, dass die Leitungsebene diese Auseinandersetzung will und unterstützt. Prävention muss in der Organisation verankert sein und sich am Wissen über Strategien von Täter_innen orientieren. Wichtig ist eine übergreifende Auseinandersetzung, die nicht nur punktuell, sondern kontinuierlich angelegt ist.

Die Einflussnahme und die Handlungsmöglichkeiten bezüglich der Eltern sind in der verbandlichen Kinder- und Jugendarbeit begrenzt. 
Eine Hinwirkung auf Inanspruchnahme von Hilfen ist nicht immer möglich. Dennoch kann versucht werden, die vertrauensvolle Basis zu den Eltern, wo sie vorhanden ist, zu nutzen. In den Fällen, wo die Voraussetzung für eine Zusammenarbeit mit den Eltern oder Sorgeberechtigten fehlt, muss der Träger sich schneller direkt an das Jugendamt wenden und dieses über die Beobachtungen informieren. Das gilt auch, wenn Fachkräfte beobachten, dass ein Kind oder ein_e Jugendliche_r, bei dem ein gewichtiger Verdacht auf Gefährdung des Wohls vorliegt, nicht mehr zu den Veranstaltungen oder Gruppen kommt (vgl. KVJS, 2009, S. 42).

\section{Vorgehen bei Verdachtsfällen}

Grob eingeteilt gibt es drei unterschiedliche Situationen bezüglich der Intervention gegen sexualisierte Gewalt innerhalb eines Verbandes. In allen drei Situationen gibt es ein paar spezifische Dinge zu beachten. Daher ist es wichtig, sich über die Art des Krisenfalls bewusst zu sein.

1. Liegt die Gefährdungdes Kindes innerhalb des familiären Umfeldes?

2. Geht die Gefährdung des Kindes von einem ehrenamtlich Mitarbeitenden aus?

3. Handelt es sich um Übergriffe unter Gleichaltrigen?

Alle drei Fälle haben die gleichen - bzw. zumindest schwerwiegende Auswirkungen auf die Betroffenen, jedoch sind unterschiedliche Handlungspläne erforderlich. Auch ist es ein Unterschied, ob das Kind oder die_der Jugendliche sich anvertraut hat oder ob man durch dessen Verhalten oder Beobachtung eine Vermutung hat.

Im Folgenden werden zwei unterschiedliche Vorgehensweisen bei einem Verdacht auf Kindeswohlgefährdung beschrieben und analysiert.

\section{Vorgehen nach Reinhold Schone}

Reinhold Schone hat auf Grundlage des $\$ 8$ a SGB VIII für freie Träger ein Verfahrensschema entwickelt, auf dem man die einzelnen Handlungsschritte verfolgen kann. 


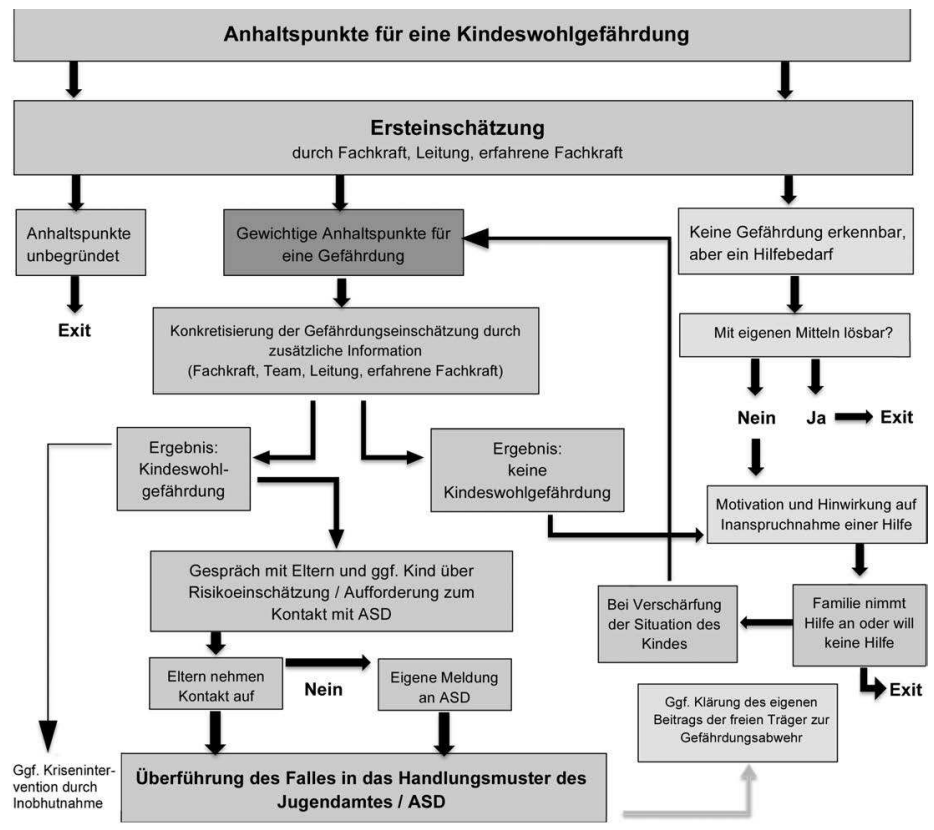

Abb. 1: Schaubild nach Expertise von Prof. Dr. Reinhold Schone (ISA, 2006, S. 97)

Der erste Schritt, nachdem der Verdacht auf eine vorliegende Kindeswohlgefährdung aufkommt, ist die Ersteinschätzung durch Fachkräfte, Leitung und erfahrene Fachkraft. In der verbandlichen Jugendarbeit muss man sich über das konkrete Vorgehen verständigen, wenn Ehrenamtliche Anhaltspunkte für eine Kindeswohlgefährdung feststellen. Wie schon im Kapitel der Begriffsklärungen beschrieben, ist es in der Regel so, dass sie sich immer zunächst an eine hauptamtlich beschäftigte Person wenden müssen. Da sie keine Fachkräfte im Sinne des Gesetzes sind, haben sie auch keine Handlungspflicht, jedoch leitet sich aus dem Selbstverständnis der Jugendverbandsarbeit der Grundsatz des Schutzes von Kindern und Jugendlichen ab und daher die Information an eine entsprechende Fachkraft. Sollte diese Ersteinschätzung ergeben, dass das geistige, körperliche oder seelische Wohl des Kindes gefährdet ist, ist der nächste Schritt ein- 
zuleiten. Dieser besteht darin, dass die Gefährdungseinschätzung konkretisiert wird, hierzu wird eine insoweit erfahrene Fachkraft hinzugezogen. Bestätigt sich hingegen der Verdacht nicht, sollte bei Bedarf auf eine Inanspruchnahme oder Hilfe hingewirkt werden, welche die Situation verbessert. Das Verfahren wäre dann erst einmal beendet.

Ergibt sich bei der zweiten Prüfung - mit der insoweit erfahrenen Fachkraft - ein verhärteter Verdacht, so sind zwei Handlungsschritte denkbar: Kontaktaufnahme mit den Eltern bzw. den Personensorgeberechtigten und dem Kind bzw. der_dem Jugendlichen oder direkt mit dem Jugendamt oder dem Allgemeinen Sozialen Dienst (ASD), wenn die Gefährdung akut ist.

In der verbandlichen Jugendarbeit sind Mitbestimmung und Selbstbestimmung wichtige Elemente, diese sollten auch bei einer Verdachtsabklärung gewahrt werden, zudem sind sie vom Gesetz her Pflicht. Der Träger sollte in diesem Schritt auf die Inanspruchnahme von Hilfen zur Abwendung der Kindeswohlgefährdung hinwirken, soweit er selber keine geeignete Hilfe anbieten kann.

Nehmen die Eltern Kontakt mit dem Jugendamt/ASD auf, dann ist für die Einrichtung der Fall abgeschlossen, wenn nicht, muss eine Meldung an den öffentlichen Träger durch die Einrichtung erfolgen.

\section{Vorgehen bei Verdachtsfällen nach Ulrich Deinet}

Eine weitere Zusammenstellung ist der von Ulrich Deinet (2006) dargestellte Verfahrensablauf für Jugendeinrichtungen; sie ist in ihrer Darstellung einfacher, inhaltlich unterscheidet sie sich jedoch kaum. 


\begin{tabular}{|c|c|c|}
\hline Ebene & Handlungsschritt & Hinweise \\
\hline $\begin{array}{l}\text { Einrichtung/ } \\
\text { Team }\end{array}$ & $\begin{array}{l}\text { 1. Beobachtung/Verdacht einer } \\
\text { Kindeswohlgefährdung } \\
\text { 2. Information der Einrichtungs- } \\
\text { bzw. Teamleitung und Doku- } \\
\text { mentation } \\
\text { 3. Kollegiale Fallberatung } \\
\text { 4. Klärung weiterer Maßnahmen }\end{array}$ & $\begin{array}{l}\text { Mögliche Anhaltspunkte ergeben sich } \\
\text { u.a. aus den Leitlinien zum Schutz- } \\
\text { auftrag bei Kindeswohlgefährdung. } \\
\text { Zur Vereinfachung der Vorgehens- } \\
\text { weise können Ablaufschemen auf } \\
\text { der Grundlage der Trägerorgani- } \\
\text { sation erstellt werden. } \\
\text { Alle unternommenen Schritte sind } \\
\text { in einem festgelegten Schema zu } \\
\text { dokumentieren. } \\
\text { Die kollegiale Fallberatung findet } \\
\text { zwischen haupt-, haupt- und ehren- } \\
\text { amtlichen bzw. zwischen ehren- } \\
\text { amtlichen Fachkräften entspre- } \\
\text { chend der Struktur statt. }\end{array}$ \\
\hline \multicolumn{3}{|c|}{$\begin{array}{c}\text { Bei übereinstimmender Einschätzung eines bohen Gefährdungsrisikos erfolgt } \\
\text { die Information an den Träger }\end{array}$} \\
\hline
\end{tabular}

\begin{tabular}{|c|c|c|}
\hline $\begin{array}{l}\text { Träger/ } \\
\text { Fachgruppe }\end{array}$ & \begin{tabular}{|l|} 
1. Kollegiale Beratung des Falles \\
in der Fachgruppe \\
2. Festlegung weiterer Hand- \\
lungsschritte: \\
a) Befriedigung des Hilfebedarfs \\
b) Hinzuziehung der insoweit \\
erfahrenen Fachkraft oder \\
weiterer Institutionen \\
3. Gespräch mit Betroffenem und \\
seinen Personensorgeberech- \\
tigten (insofern die Hilfe da- \\
durch nicht gefährdet wird) \\
\end{tabular} & $\begin{array}{l}\text { Unter Nutzung eventuell vorhan- } \\
\text { dener fachlicher Kompetenzen } \\
\text { kann die Beratung des Falles inner- } \\
\text { halb des Trägers z.B. in der »Fach- } \\
\text { gruppe Kindeswohlgefährdung« } \\
\text { des Trägers weiter erfolgen. Sollte } \\
\text { dies nicht möglich sein, ist eine ex- } \\
\text { terne insoweit erfahrene Fachkraft } \\
\text { hinzuzuziehen. }\end{array}$ \\
\hline $\begin{array}{l}\text { Kontaktauf } \\
\text { nahme mit } \\
\text { Jugendamt }\end{array}$ & $\begin{array}{l}\text { Information des Jugendamtes, wenn } \\
\text { die angebotenen Hilfen nicht zu } \\
\text { einer Veränderung führen }\end{array}$ & $\begin{array}{l}\text { Die Kontaktaufnahme zum Ju- } \\
\text { gendamt erfolgt über festgelegte } \\
\text { Personen des Trägers. }\end{array}$ \\
\hline ! & \multicolumn{2}{|c|}{$\begin{array}{l}\text { Alle Schritte dieses Ablaufschemas sind zu dokumentieren. Die Hinzu- } \\
\text { ziehung weiterer Personen zur Fallberatung bzw. bei Weitergabe von } \\
\text { Information muss unter Berücksichtigung datenschutzrechtlicher Be- } \\
\text { stimmungen erfolgen. }\end{array}$} \\
\hline
\end{tabular}

Tab. 2: »Expertise: Schutzauftrag bei Kindeswohlgefährdung - Kinder- und Jugendarbeit« (nach Deinet, 2006)

Ulrich Deinet ergänzt die Handlungsschritte mit Hinweisen, die für die Einrichtungen bzw. das Team hilfreich sein können. Eine erste Handlung nach dem Verdacht einer Kindeswohlgefährdung beschreibt er in der Information der Einrichtungs- bzw. der Teamleitung. In einer kolle- 
gialen Fallberatung sollen weitere Maßnahmen abgeklärt werden. Diese kollegiale Fallberatung wird entsprechend der verbandsinternen Struktur mit haupt- und ehrenamtlich Beschäftigten durchgeführt. Wird in dieser Fallberatung das Gefährdungsrisiko als hoch eingeschätzt, erfolgt die Information an den Träger.

Sind in der Einrichtung fachliche Kompetenzen, so kann der weitere Schritt innerhalb der Trägerschaft erfolgen. Ist das nicht der Fall, hat der Träger hierfür eine externe, insoweit erfahrene Fachkraft hinzuzuziehen. Nach einer weiteren kollegialen Beratung in der Fachgruppe erfolgt das Gespräch mit der_dem Betroffenen und den Eltern bzw. Personensorgeberechtigten, sofern die Hilfe dadurch nicht gefährdet wird.

Wird diese Hilfe nicht angenommen und vonseiten der Eltern die Inanspruchnahme der Hilfen beim Jugendamt nicht in die Wege geleitet, ist der Kontakt zum Jugendamt durch eine festgelegte Person aus der Einrichtung aufzunehmen.

Deinet weist darauf hin, dass alle Schritte zu dokumentieren sind. Bei der Weitergabe der Daten sind die datenschutzrechtlichen Bestimmungen zu beachten (vgl. Deinet, 2006).

\section{Vergleich der Modelle zum Vorgehen bei Verdachtsfällen in der verbandlichen Jugendarbeit}

Die Handlungsaufforderung des $\$ 8$ a greift erst zu dem Zeitpunkt, wenn sich Hinweise auf eine Kindeswohlgefährdung ergeben. Es ist nicht Aufgabe und Pflicht von Fachkräften oder gar ehrenamtlich Mitarbeitenden, sich detektivisch mit einem »kriminalistischen « Spürsinn auf die Suche nach Fällen von Kindeswohlgefährdung zu machen. Jedoch sollten einem Verdacht und »Bauchgefühl « nachgegangen werden.

Wenn Mitarbeitende einen Verdacht haben oder ihnen ein konkreter Fall anvertraut wird, entstehen vielerlei Gefühle, mit denen man zurechtkommen muss. Entscheidend für einen guten Verlauf beim weiteren Vorgehen sind klare Handlungsvorgaben im Jugendverband. Daher ist es wichtig, dass Jugendverbände sich im Vorfeld Gedanken dazu machen, was in einem Verdachtsfall oder in einem konkreten Fall von Kindeswohlgefährdung getan werden muss. Auch hier ist die Transparenz ein wichtiger Faktor bei der Aufklärung. 
Im Vergleich der Modelle fällt auf, dass Deinet den Ablauf auf die konkrete Situation in der Kinder- und Jugendarbeit bezieht. Er macht in seinen Hinweisen Vorschläge für die Umsetzung. Ein solcher Vorschlag ist die Erstellung eines Ablaufschemas als Grundlage und Vereinfachung der Vorgehensweise. Deinet bezieht explizit die betreffenden ehrenamtlich Mitarbeitenden in die Fallbesprechung ein und weist damit auf die Struktur der Kinder- und Jugendarbeit hin. Beim weiteren Vorgehen setzt er die durch das KICK gewollte Stärkung der freien Träger in ihren Möglichkeiten um: Er stellt durch den Hinweis auf eine trägerinterne Fachgruppe zur Kindeswohlgefährdung die damit verbundene Kompetenzen der Träger in den Vordergrund (vgl. ebd.).

Meines Erachtens ist das Modell von Ulrich Deinet eine geeignete Vorlage für die Umsetzung der Handlungsschritte für die verbandliche Jugendarbeit. Zur Dokumentation der Beobachtungen und Gespräche im konkreten Fall ist ein festgelegtes Schema sinnvoll (siehe Anhang A7).

Zur Ergänzung des Modells von Deinet sind die Hinweise von Beate Steinbach sehr hilfreich. Sie weist auf die besonderen Strukturmerkmale des ehrenamtlichen Kontextes hin, die mittels einer Risikoanalyse herausgearbeitet werden müssen und im Handlungskonzept individuell Beachtung finden sollten.

Dazu zählen für sie unter anderem:

$>$ Voraussetzung für ehrenamtliches Engagement ist ein niedrigschwelliger, einladender Zugang, der aber auch Risiken birgt, insbesondere, wenn es einen Mangel an ehrenamtlich aktiven Personen gibt.

$>$ Kleine Jugendorganisationen und -initiativen oder auch selbstorganisierte Jugendgruppen verfügen eher über informelle Strukturen als klare Leitungs- und Entscheidungsstrukturen, was bezüglich des Aufgreifens von Bedürfnissen und Interessen der Zielgruppen von Vorteil ist, jedoch auch das Risiko des Missbrauchs enthält.

$>$ Motivation des Ehrenamtes liegt in den sozialen Kontakten, den Freundschaften und in den gemeinsamen Zielen, der Identifikation mit dem Jugendverband. Im Gegensatz zum beruflichen Umfeld ist die Grenze zwischen Ehrenamt und Privatleben nicht immer klar, da das Ehrenamt als Teil des Privatlebens gesehen wird (vgl. Steinbach, 2015, S. 188f.). 
Einige Verbände und (Kreis/Landes-)Jugendringe haben Handlungsleitfäden entwickelt. Eine Zusammenstellung empfehlenswerter Handlungsleitfäden befindet sich im Anhang A8. Für größere Verbände ist es sinnvoll, auf dieser Grundlage einen eigenen Handlungsleitfaden zu entwickeln, der auch verbandsspezifische Besonderheiten beinhaltet, wie Ansprechpersonen und Rufnummern. Dass dieser Leitfaden sich dann auch zum Einsatz bei den Schulungen eignet, ist ein Grund mehr, hier ein Augenmerk darauf zu haben.

Im Anhang A8 gibt es Anregungen, wie ein solcher Handlungsleitfaden aufgebaut sein kann und welche weiteren Inhalte sinnvoll sind.

\section{Vorgehen beim Verdacht auf Täter_innen in den eigenen Reihen}

Für die verbandliche Jugendarbeit ist das Thema des Umgangs mit »Täter_innen in den eigenen Reihen « ein relevantes und mit Unsicherheit behaftetes. Daher ist neben einem Handlungsplan bei Verdacht auf Kindeswohlgefährdung immer auch das Thema Grenzverletzungen und Übergriffe durch Mitarbeitende zu bedenken, und wie im Falle einer Vermutung oder Beobachtung vorzugehen ist.

Neben den allgemeinen Verhaltensweisen ist Folgendes für einen Krisenplan hilfreich:

> unverzügliche Kontaktaufnahme zur Ansprechperson innerhalb des Verbandes, sofern benannt. Sollte es sich um eine Täterschaft einer hauptamtlichen Person handeln, dann Kontaktaufnahme zum/zur Vorgesetzten.

$>$ Ziel muss auf jeden Fall sein, die Übergriffe zu beenden, ohne in vorschnellen Aktionismus zu verfallen (z. B. verdächtige Person nicht mehr alleine mit Schutzbefohlenen lassen).

> Schon im Vermutungsfall müssen die Verantwortlichen deutlich machen, dass sie auf der Seite des Opfers stehen und mit Konsequenzen gegenüber dem Täter/der Täterin reagieren.

$>$ Was passiert bei einer falschen Verdächtigung? Wie sieht das Rehabilitationsverfahren aus? 


\section{Vorgehen bei Grenzverletzungen und Übergriffen unter Gleichaltrigen}

In der verbandlichen Jugendarbeit sind Mitarbeitende, ob ehren- oder hauptamtlich, vermutlich öfters mit der Thematik der Peergewalt konfrontiert als mit Übergriffen durch erwachsene Bezugspersonen.

Der Umgang in solchen Situationen ist ein Thema, welches sich in der Haltung widerspiegelt. In Handlungsfäden und Empfehlungen ist daher der grenzachtende und respektvolle Umgang untereinander hervorzuheben. Auch die Frage nach den Gruppenregeln ist hier zu stellen.

Mitarbeitende müssen bei Gewahrwerden von Grenzverletzungen oder Übergriffen unter Gleichaltrigen in ihrem Verantwortungsbereich eindeutig Stellung beziehen und die Situationen angemessen und schnell klären (vgl. Enders, 2012). Auch hier gilt es, je nach Schwere des Vorfalls eine fachlich versierte Person hinzuzuziehen. Die beteiligten Kinder und Jugendlichen sollten nicht gemeinsam und nur mit Bedacht befragt werden. Dabei sind die Schamgrenzen zu beachten. Eltern sind zu informieren. Für alle beteiligten Kinder und Jugendliche sowie Eltern sind Unterstützungsangebote aufzuzeigen.

\section{Beauftragung von ehrenamtlich Mitarbeitenden}

Die Transparenz im Verband beinhaltet auch den offenen Umgang bei der Beauftragung von Mitarbeitenden. Eine ehrenamtliche Mitarbeit im Verband ist geprägt von Freundschaft und Beziehung zu den dort anwesenden Menschen und spielt daher eine größere Rolle als bei beruflichen Kräften. Die Motivation, sich zu engagieren, ist so vielfältig, wie Menschen selbst verschieden sind: Der Wunsch nach neuen Kontakten, Hilfsangebote für Bedürftige, Selbstverwirklichung und berufliche (Neu-)Orientierung, die Wahrnehmung sozialer Verantwortung und aktiver Mitbestimmung und in den kirchlichen Verbänden auch eine religiöse Grundhaltung kann Motivation für ein Engagement sein. Dabei spielen egoistische und auch altruistische Motive eine Rolle. Im Bereich der Jugendlichen ist die Motivation im freundschaftlichen Bereich. Dort, wo sich die Freunde aufhalten oder engagieren, wird man auch selbst tätig. Oft ist die ideelle Bindung und die Identifikation mit dem Jugend- 
verband ein Grund, sich zu engagieren. Die Zugänge zum Ehrenamt sind ebenso vielfältig und reichen von der traditionellen Verwurzelung im Verband der Familie über Freundschaften bis hin zu einem interessengeleiteten Suchen im Erwachsenenalter. Durch die hohe zeitliche Inanspruchnahme der Jugendlichen durch die Schule ist zu beobachten, dass sich die traditionellen Ehrenamtskarrieren auflösen. So zeigen die Ergebnisse der 17. Shell Jugendstudie einen Rückgang im gesellschaftlichen Engagement; 34 Prozent der Jugendlichen setzen sich »oft « für andere im Alltag ein. Eine langfristige, regelmäßige Mitarbeit im Verband wird immer seltener und »bei ehrenamtlichen Mitarbeiter/-innen besteht eine verhältnismäßig hohe Fluktuation « (Fegert \& Wolff, 2015, S. 189). Das hat auch Auswirkungen auf die Begleitung der jungen Menschen im Ehrenamt. Die kontinuierliche Begleitung vom Jugendalter bis ins Erwachsenenalter wurde von einer »Patchwork-Begleitung « abgelöst, die einen Beziehungsaufbau zu der jeweiligen Person erschwert. Hinzu kommt die hohe Mobilität durch Ausbildung oder Studium. Die veränderte Struktur der Ehrenamtlichkeit hat Auswirkungen auf die Personen, die sich im Jugendverband engagieren. Dadurch, dass sich vermehrt ältere für ein ehrenamtliches Engagement interessieren, besteht auch die Gefahr, dass unter ihnen Menschen sind, die pädophile Neigungen haben und in Gefahr sind, Missbrauchstäter_innen zu werden. Sie suchen sich bewusst (oder auch unbewusst) eine Tätigkeit im Bereich der Jugendarbeit.

Es liegt mir fern, zu stigmatisieren und alle in der Jugendarbeit Tätigen als potenzielle Täter_innen unter Generalverdacht zu stellen. Jedoch kann nicht davon ausgegangen werden, dass nur Personen mit pädophilen Neigungen zu Täter_innen werden. So gibt es genügend Studien, die dies belegen. Bei den Täter_innen gibt es neben jenen mit pädophilen (oder hebephilen - also mit sexueller Ansprechbarkeit für das frühe Jugendalter) Neigungen auch Personen, die diese sexuelle Präferenzstörung nicht vorweisen und sexuellen Missbrauch als »Ersatzhandlung « begehen. Frauen als Täterinnen finden sich eher in der zweiten Gruppe ${ }^{7}$ (vgl. Fegert et al., 2015, S. 110f.).

7 Im Präventionsnetzwerk »Kein Täter werden« haben sich bisher nur wenige Frauen gemeldet, die angaben, pädophile Neigungen zu haben; bei einer konnte dies diagnostisch bestätigt werden (vgl. https://www.kein-taeter -werden.de/story/52/3852.html). 
Eine differenziertere Betrachtung von Täter_innen ist an dieser Stelle zu umfangreich und kann in den Quellen gut nachgelesen werden.

Als Strategien von Täter_innen sind hier jedoch benannt, dass Täter_innen planvoll vorgehen und sich an ihre potenziellen Opfer herantasten; häufig sind diese Menschen sehr einfühlsam und fürsorglich, auch gegenüber den Teamkolleg_innen. Sie sind sich weder eines Unrechts bewusst noch willig, ihre Tat einzusehen. Werden sie auf Grenzüberschreitungen angesprochen, können sie beleidigt reagieren oder wechseln ihren Einsatzort. Was besonders schwierig in der Einschätzung ist, ist, dass sie oft sehr umfangreiche Erfahrungen im pädagogischen Bereich vorweisen können, auch gepaart mit kreativen oder handwerklichen Fähigkeiten. Ideale Ehrenamtliche also, die besonders geeignet erscheinen und über hohe Kommunikations- und Empathiefähigkeit verfügen. Die Methoden pädophiler Mitarbeiter, die gefährdet sind, Missbrauchstäter zu werden, sind oftmals sehr subtil (vgl. Conen, 2006, S. 57f.; Fegert \& Wolff, 2006, S. 310). So gilt es, Personen, die zu Taten neigen, im Vorfeld abzuschrecken.

Die weitere Auseinandersetzung mit Strategien von Täter_innen führt in diesem Zusammenhang zu weit.

Nach meinen Recherchen gibt es kaum Literatur, die sich mit der Beauftragung von Ehrenamtlichen in Bezug auf den Schutz vor Kindeswohlgefährdung beschäftigt. Marie-Luise Conen führt geeignete Aspekte für die Personalauswahl von hauptamtlichen Kräften auf, die ich aber auf Ehrenamtliche als nur eingeschränkt anwendbar ansehe. So weist sie darauf hin, dass sich Freundschaften hinderlich auf die Prävention von Kindeswohlgefährdung durch Professionelle auswirken, da hier durch die enge Vertrautheit und eventuelle Abhängigkeiten eine Vertuschungsgefahr bestehe; ebenso rät sie Leitungspersonen, die Bildung von Insidergruppen abzumildern bzw. zu verhindern, da diese sich »korrumpieren und Vorgehensweisen entwickeln, in denen gegenseitige Verpflichtungen Grenzverletzungen nicht mehr ansprechbar werden lassen « (Conen, 2006, S. 62). Diesen Aspekt sehe ich im Zusammenhang mit Ehrenamtlichen als nicht durchführbar. Ehrenamtliche Mitarbeit erwächst häufig aus Gruppenzugehörigkeit und bedeutet auch, im Rahmen der ehrenamtlichen Arbeit (und darüber hinaus) Freundschaften zu pflegen. 
Ehrenamtliche engagieren sich nicht über Stellenanzeigen und müssen sich in der Regel auch keinem Auswahlverfahren unterziehen. Einen Ansatz halte ich für sehr gut umsetzbar: Wenn sich Menschen ehrenamtlich in der verbandlichen Jugendarbeit engagieren möchten, »sollten schriftliche Informationen der Einrichtung bzw. Informationsmaterial von Verbänden eingebracht werden zu Kinderrechten, Warnsystemen sowie Standards der Einrichtung (soweit vorhanden) zu diesem Thema « (ebd., 2006, S. 58). Diese Standards sollten besprochen werden. Durch ein solches Verfahren kann es aber auch sein, dass eventuell gute Mitarbeiter_innen abgeschreckt werden. Daher ist es notwendig, diesen Bereich sensibel, aber bestimmt auszugestalten. Ein wichtiges Instrument sind die Schulungen für Ehrenamtliche. Gerade auch im Bereich der Freizeiten, in denen oft langährige Mitarbeitende engagiert sind, ist die regelmäßige Thematisierung und damit die Haltungsüberprüfung wichtig. Dennoch muss man festhalten: Keine noch so kritische und sorgfältige Auswahl von Mitarbeitenden kann letztendlich eine Grenzüberschreitung verhindern. Aber ein genaues Nachund Hinterfragen dient nach außen vor allem der Abschreckung von Missbrauchstäter_innen und nach innen der Auseinandersetzung und Sensibilisierung der Mitarbeitenden (vgl. ebd., S. 57).

Ein präventiver Ansatz ist es, eine Rückmeldungskultur einzurichten, bei der ehrenamtlich und beruflich Mitarbeitende zusammenarbeiten. Denn wenn ich es gewohnt bin, dass ich konstruktive Rückmeldung zu meiner Arbeitsweise erhalte und im Team diese auch an andere geben kann, fällt es mir leichter, auch unangenehme Beobachtungen kritisch zu äußern und zu hinterfragen. Denn »fehlende Rückmeldungen an MitarbeiterInnen [können] ein [...] Grund sein, der bei MitarbeiterInnen zu grenzverletzendem Verhalten beiträgt « (ebd., S. 56). Ähnlich beschreiben es auch Markus Schnapka und Martin Stroppel: »Wenn etwas schief läuft, gibt es in allen Teams des Arbeitslebens zunächst die Neigung zur Verdrängung, zum Nicht-Wahrnehmen, zum >Das-regeltsich-schon-von-selbst < « (Schnapka \& Stroppel, 2006, S. 173).

Eine solche Gesprächskultur im Verband ist nicht leicht zu etablieren. Es benötigt Mut und Entschlossenheit, sich vielleicht gegen Traditionen zu stellen und eine klare Position zu beziehen, vor allem in einer Zeit, in der man froh über jeden ist, der sich freiwillig im Verband engagiert. Dennoch kann durch eigenes Reflektieren und Weiterent- 
wicklung eine offene Gesprächskultur und ein offener Umgang mit dem Thema und eigenen Unsicherheiten entwickelt werden. Hilfreich ist hier sicher ein strukturierter Ablaufplan für solche Gespräche und Schulungseinheiten. Der_die durchführende Mitarbeiter_in sollte sich hier gut einarbeiten und sich selbst bewusst entsprechende Fragen stellen und das eigene Handeln reflektieren. Auch konkrete Situationen für Gesprächsanlässe in der Mitarbeiter_innenbildung sind hier sicher hilfreich, mit den Ehrenamtlichen ins Gespräch zu kommen. Denn Ehrenamtliche brauchen in ihrer Arbeit ein Gegenüber, das mit ihnen Gespräche über den Umgang mit Grenzsituationen und dem Umgang mit den Kindern und Jugendlichen führt.

Als ein geeignetes Instrument sehe ich hier besonders die Verhaltenskodizes mit Verpflichtungserklärung an, wie sie inzwischen in vielen Verbänden Standard sind. Sie sollten besprochen werden und darüber hinaus kann dieses Gespräch dazu genutzt werden, offen über die eigenen Empfindungen gegenüber sexueller Gewalt zu sprechen. Für eine umfassende Auseinandersetzung ist dies jedoch nicht ausreichend. Conen bewertet das offene Ansprechen als nicht zu unterschätzenden Abschreckungseffekt bei Menschen, die potenzielle Missbrauchstäter_innen sein könnten, » auch wenn es einige Überwindung kostet, diese Fragen so klar und deutlich zu stellen «(Conen, 2006, S. 59).

Im Evaluationsbericht zum Bundeskinderschutzgesetz zeigte sich, dass die Einsicht in Führungszeugnisse in einigen Fällen aufdeckend war und ein Tätigkeitsausschluss wurde ausgesprochen.

Dennoch muss man sich bewusst sein, dass sie lediglich nur dann etwas über Täterschaft aussagen, wenn eine Verurteilung vorliegt. »Je jünger Personen sind, desto geringer ist die Wahrscheinlichkeit, über ein Führungszeugnis Hinweise auf Ungeeignetheit im Sinne des §72a SGB VIII aus den Führungszeugnissen zu entnehmen « (DBJR, 2006, S. 1).

\section{Ausbildung von ehrenamtlich Mitarbeitenden}

Die zentrale Aufgabe der Jugendverbände liegt in der Verbesserung der Schulungen in Bezug auf den Schutzauftrag. In fast allen Schulungskonzepten der großen Jugendverbände findet man thematische Einheiten 
zu Schutz und Prävention. Oft sind ganze Schulungsordner mit diesem Thema gefüllt. Besonders auch der Bereich der Freizeitleiterschulung ist hier im Blick der Jugendverbände.

Es geht allerdings nicht um die Ausbildung von Experten in Sache Kindeswohlgefährdung, wie auch Voigts ausführt, denn

»[e]hrenamtliche Mitarbeitende in der Jugendarbeit sind keine dezidierten Expert/innen für die Erkennung der Gefährdung des Kindeswohls, wie es zum Beispiel die Fachkräfte der Erziehungshilfe freier Träger oder des ASD der Jugendämter sind. Es kann und darf auch nicht Anspruch sein, sie zu den Experten für die Gefährdung des Kindeswohls zu machen $\ll(D B J R, 2006$, S. 2).

Jugendleiter sollten aber für die Anzeichen einer Kindeswohlgefährdung sensibilisiert werden; das hilft im Notfall, mit einer konkreten Situation umzugehen und sich fachlichen Rat zu holen.

Die Ansprüche der Schulungskonzepte an die ehrenamtlich Mitarbeitenden sind recht hoch und können bei jungen Mitarbeitenden zu einer Überforderung führen, da sie nicht selten noch in der eigenen Entdeckung von Sexualität und sexueller Orientierung sind. Daher ist eine differenzierte Betrachtung der Schulungseinheiten je nach Alter und Erfahrung der ehrenamtlich Mitarbeitenden notwendig.

Die Aufgabe von Schulungskonzepten muss neben der Wissensvermittlung im Verdachtsfall auch insbesondere die Entwicklung einer grenzachtenden und respektvollen Haltung sein. Dies ist nur durch eine durchgehende Thematisierung der unterschiedlichen Aspekte von sexualisierter Gewalt und deren Prävention zu entwickeln.

Im Hintergrundpapier des Deutschen Bundesjugendrings zum Stand der Entwicklung und Umsetzung umfassender Präventionsansätze in der Jungend(verbands)arbeit wird darauf hingewiesen, dass der »Prozess der Entwicklung von Präventionsmaßnahmen [...] in den Jugendverbänden bereits seit ca. 10 Jahren « (ebd., S. 1) läuft. Bei vielen Trägern finden sich konkrete Präventionskonzepte, die seit 2003 erprobt und weiterentwickelt werden. Besonders hervorgehoben werden kann das Konzept »Prä Tect « vom Bayerischen Landesjugendring (BJR).

Dieses Projekt gehört zu den ältesten Präventionsprojekten gegen sexuelle Gewalt und ist Grundlage für so manch anderes Präventions- 
projekt in der verbandlichen Jugendarbeit. Es startete 2003 als Modellprojekt. Seit 2006 gibt es daran anschließend die Fachberatung im Bayerischen Landesjungendring. Oberste Leitziele von PräTect sind, das Feld der Jugendarbeit für potenzielle Täter_innen unattraktiv zu machen und ein »Netz der Sicherheit « für Jungen und Mädchen zu entwickeln. Dies soll dadurch erreicht werden, dass auf allen Ebenen der verbandlichen Jugendarbeit präventives Handeln verankert wird. $\mathrm{Zu}$ den Grundsätzen gehört, dass Prävention als Qualitätsmerkmal in der Arbeit mit Kindern und Jugendlichen angesehen wird, Erwachsene in die Verantwortung für Prävention genommen werden und die Notwendigkeit gesehen wird, dass verbandliche Jugendarbeit ein individuelles, auf das eigene Profil abgestimmtes Präventionskonzept benötigt. Dieses soll partizipativ im Prozess entwickelt werden. Prä Tect ist keine Zusammenstellung von Rezepten, sondern soll durch individuelle Anpassung an die Situation für jeden Verband zurechtgeschnitten werden. So wird auf der Homepage (www.praetect.de) unter anderem Folgendes angeboten:

$>$ Hilfe bei der Organisation von Schulung und Fortbildung und Vermittlung von kompetenten Referent_innen

> Vermittlung von Kontakten zu Beratungsstellen

$>$ Info zu möglichen Präventions- und Schutzmaßnahmen in der Jugendarbeit

$>$ Info und Beratung zu einzelnen Themen (z. B. Sexualpädagogik, geschlechtsspezifische Jugendarbeit ...)

$>$ Qualifizierungsreihe für Leitungs- und Fachkräfte

$>\quad$ Schulung für Vertrauenspersonen gegen sexuelle Gewalt

Auf dem »Markt « gibt es inzwischen eine Vielzahl von guter Literatur und Vorschläge für die Umsetzung des Schutzauftrages in der verbandlichen Jugendarbeit. Auch wenn es viele verschiedene Gruppenstundenvorschläge und andere Methodenhandbücher gibt, sollte mit einer Umsetzung immer sensibel umgegangen werden. »So kann beispielsweise die gut gemeinte und methodisch durchgeplante Einheit zur eigenen Körperlichkeit zum Missbrauch werden, wenn die individuellen Grenzen Einzelner übertreten werden « (EJB, 2004, S. 27). Das erfordert eine gewisse innerliche Auseinandersetzung mit dem Thema und dem eigene Erleben der Methoden. 
Um eine gute und qualitativ hochwertige Auseinandersetzung im Jugendverband zu gewährleisten, ist die fachliche Auseinandersetzung und Fortbildung von hauptamtlichen Personen sowie die Einbindung von (und Kooperation mit) Fachberatungsstellen in die Schulungsarbeit wichtig.

Einen wichtigen Hinweis für die Durchführenden von Mitarbeiter_innenschulungen sehe ich darin, dass derjenige, der die Themen »Sexualität, sexuelle Gewalt, Kindeswohlgefährdung « behandelt, bereit sein muss, sich selbst damit vertieft auseinanderzusetzen. Eine Konfrontation mit konkreten Fällen ist dabei jederzeit möglich. Dabei ist zu beachten, dass alle Beteiligten die individuellen persönlichen Grenzen respektieren, die durch Überforderung, unverarbeiteter Betroffenheit oder Hilflosigkeit dem Thema gegenüber auftreten können. Die Einbindung von Sexualität im Kontext der sexuellen Gewalt in der Mitarbeiter_innenbildung des Jugendverbandes steht und fällt mit der Offenheit und Natürlichkeit der Leitungsperson. Es ist daher Grundsatz, dass keine Leitungsperson gezwungen werden kann, dieses Thema zu behandeln. Hier gilt, wie im ganzen Themenbereich, die eigenen und fremden Grenzen zu respektieren. Dennoch ist es für ein gutes Schutz- und Präventionskonzept wichtig, dass Gelegenheiten geschaffen werden, diese Themen zu besprechen und eine verbandsinterne Haltung entwickelt wird. Ganz sicher müssen sich hauptamtlich Tätige in der verbandlichen Jugendarbeit und gegebenenfalls auch ehrenamtliche Leitungskräfte in diesem Bereich weiterbilden. Hier müsste bei den öffentlichen Jugendhilfeträgern ein Bewusstsein geschaffen werden. So weist auch Beate Steinbach daraufhin, dass es gerade für den Bereich der verbandlichen Jugendarbeit spezielle Fort- und Weiterbildungsangebote für Fach- und Leitungspersonen geben muss. Bei Weiterbildungen in anderen Bereichen der Jugendhilfe werden die Besonderheiten des Ehrenamtes wenig thematisiert, weil sie »kaum eine Rolle spielen « (Steinbach, 2015, S. 195).

Die Einbindung des Erfahrungshorizontes, des Alters und der Lebenswelt der Teilnehmenden bei JuLeiCa-Seminaren ist im Bereich des Kinder- und Jugendschutzes besonders wichtig, um eine Überforderung zu vermeiden. Sensibilisierung und Informationen über Hilfsangebote stehen an erster Stelle. Mit zunehmendem Alter und Verantwortung im Verband sind die Ausbildungsinhalte dahin gehend zu präzisieren 
und abzustimmen, dass sich entsprechende Kompetenzen im Bereich Kinderschutz entwickeln. So braucht ein Gruppenleiter andere Kompetenzen als ein ehrenamtlicher Teamleiter einer Freizeit mit entsprechender Verantwortung. 


\section{Konzeptionelle Überlegungen zur Einbindung des Schutz- auftrages in die Ausbildung von ehrenamtlich Mitarbeitenden}

Bei der Umsetzung des Schutzauftrages in der verbandlichen Jugendarbeit geht es einerseits darum, Kindern beizustehen, deren Wohl gefährdet ist, und andererseits aber auch um den Schutz vor Mitarbeiter_innen, die ihre Macht gegenüber den ihnen anvertrauten Kindern und Jugendlichen zur Befriedigung eigener (sexueller) Bedürfnisse missbrauchen.

Die JuLeiCa-Standards von Baden-Württemberg beinhalten die Auseinandersetzung mit dem Schutzauftrag in zwei Themenbereichen: 1. Kenntnis über den Schutzauftrag innerhalb dem Themengebiet der rechtlichen Rahmenbedingungen von Jugendverbandsarbeit (vgl. LJRBW, 2009, Kapitel 5.2.3)

2. Einfügung eines neuen Themengebiets innerhalb der JuLeiCaStandards, der sich mit Kindern und Jugendlichen in Notsituationen beschäftigt. Als konkretes Ziel wird hier benannt: »Die Jugendleiter_innen haben sich mit dem Thema Kindeswohlgefährdung beschäftigt. Sie sind darüber informiert, an wen sie sich wenden können, wenn sie mit einer Gefährdung des Kindeswohls konfrontiert werden « (ebd., S. 14). In den Erläuterungen werden die Inhalte der Schulung sehr weitgehend beschrieben. So sollen neben den Handlungsschritten Jugendleiter auch Kenntnisse über die Aufgaben des Jugendamtes erhalten. Das ist sicher für erwachsene ehrenamtlich Mitarbeitende interessant und könnte neben der Auseinandersetzung mit Wertehaltungen und den Ursachen von Gefährdung unter Allgemeinbildung/Erwachsenenbildung ein Thema für die Vertiefungsseminare sein. 
Das Thema der Kindeswohlgefährdung sollte in den JuLeiCa-Schulungen meines Erachtens in bestehende Module eingebunden werden, um den Gedanken des Kinderschutzes als Querschnittsaufgabe zu unterstreichen.

In der verbandlichen Jugendarbeit sind die JuLeica-Schulungen oftmals in Form von Basis- und Aufbauseminaren konzipiert. In der Regel sind ehrenamtlich Mitarbeitende beim Besuch des Basisseminars 15 Jahre alt. Schwerpunkt dieses Seminars über fünf Tage ist das praktische Tun: Spielpädagogik, Gruppengründung, Programmplanung und Aufsichtspflicht. Ein Jahr später besuchen die meisten von ihnen das fünftägige Aufbauseminar. Vertiefende Seminare mit reflektierendem Charakter der Leitungsrolle sind Inhalt der Schulung. Nach diesem Seminar und dem Besuch eines Erste-Hilfe-Kurses erhalten die ehrenamtlich Mitarbeitenden die Jugendleiter-Card. Des Weiteren gibt es Vertiefungsseminare, die sich intensiver mit bestimmten Themen beschäftigen. Diese sind als weitere Qualifizierung Ehrenamtlicher gedacht und richten sich an junge Erwachsene, die die Basisschulung besucht haben. Aufgrund der besagten Altersstruktur und der Tatsache, dass junge Mitarbeitende in der Regel nicht alleine Gruppen und Freizeiten leiten, sondern dies mit erfahrenen Mitarbeitenden tun, sehe ich es gegeben, dass die Thematisierung des Schutzauftrages im Aufbauseminar verortet wird. Im Basisseminar sollte das Thema jedoch auch nicht verschwiegen werden. So ist das Aufgreifen des Leitungsverständnisses (Aufmerksamkeit dem Einzelnen gegenüber, respektvoller Umgang miteinander, Mitbestimmung etc.) und der Grundhaltung gegenüber den anvertrauten Kindern eine Primärprävention, die darauf hinzielt, die Ausgestaltung der Arbeit vor Ort dahin gehend umzusetzen, dass sie wertschätzend und fördernd ist.

Da die rechtlichen Grundlagen mit Aufsichtspflicht, Haftung etc. Inhalt des Basisseminars sind, sehe ich es als notwendig an, im Aufbauseminar noch eine kurze Rechtseinheit unterzubringen, die den Schutzauftrag zum Inhalt hat.

Wesentliche Ziele für eine Einbindung in die bestehenden Module der Basisbildung sind:

\footnotetext{
$>$ Kenntnisse über den Schutzauftrag

$>$ Sensibilisierung
} 
- Auseinandersetzung mit möglichen Anhaltspunkten für die Kindeswohlgefährdung

$>$ Wissen über die notwendigen Handlungsschritte

Diese Ziele gelten in der Form auch für die aufgeführten Bausteine für die Vermittlung des Themas bei Teamtreffen und thematischen Abenden für Jugendgruppen.

Ein sehr wichtiges Angebot der Jugendverbände sind Freizeiten. Hier ist ein großer Bereich für ehrenamtliches Engagement vorhanden. Viele Freizeiten werden ohne Beteiligung von hauptamtlichen Kräften durchgeführt, daher sind Schulungsmodule gerade in diesem Bereich wichtig, um das Verständnis und die Regeln des Verbandes zu verankern.

Im Folgenden versuche ich verschiedene Themenbereiche aufzugreifen, die sich für die Integration in bestehende Module eignen, und hilfreiche Methoden zu benennen. Im Anhang sind die Methoden zu finden, die für ein Verständnis hilfreich sind. Bei anderen ist die entsprechende Literatur angegeben. In der Regel haben die Teilnehmenden bei den Aufbauseminaren etwas Gruppenleitererfahrung. Durch ihr doch recht junges Alter kann nicht von einer umfassenden Kenntnis und Erfahrung ausgegangen werden. Die Wahrscheinlichkeit, dass von (sexualisierter) Gewalt betroffene Jugendliche in den Seminaren sind, ist gegeben und sollte bewusst sein. Wie es gelingt, das Thema angemessen aufzugreifen, ohne die jungen Ehrenamtlichen zu überfordern, ist bei jeder Gruppenzusammensetzung erneut zu prüfen und zu beantworten. Hier ist die Professionalität der Seminarleitung gefragt.

In den Seminaren des Aufbauseminars geht es primär um eine Sensibilisierung der teilnehmenden Ehrenamtlichen und das Kennenlernen von Abläufen bei Vorfällen.

\section{Bausteine für den Bereich Entwicklungspsychologie}

Der Bereich der Entwicklungspsychologie eignet sich für die Bewusstmachung von kindlichen Bedürfnissen, die Betrachtung ihrer Lebens- 
welt und die altersgemäße sexuelle Entwicklung, soweit die Kenntnisse für die Sensibilisierung zur Wahrnehmung des Schutzauftrags hilfreich sind. So können schon vorhandene Seminareinheiten um diese Aspekte erweitert werden:

\section{Kindliche Bedürfnisse}

Ziel für die Einbindung der kindlichen Bedürfnisse:

$>$ Den Seminarteilnehmenden (TN) sind die vielfältigen kindlichen Bedürfnisse bekannt.

\begin{tabular}{|c|c|c|c|}
\hline Zeit & Inhalt & Methode & Material \\
\hline $30^{\prime}$ & $\begin{array}{l}\text { Bedürfnisse von Kindern } \\
\text { und Jugendlichen erkennen: } \\
\text { - körperliche Bedürfnisse } \\
\text { - Schutzbedürfnisse } \\
\text { - Bedürfnis nach einfühlen- } \\
\text { dem Verständnis und } \\
\text { sozialer Bindung } \\
\text { - Bedürfnisse nach Wert- } \\
\text { schätzung } \\
\text { - Bedürfnisse nach Anre- } \\
\text { gung, Spiel und Leistung } \\
\text { - Bedürfnisse nach Selbst- } \\
\text { verwirklichung }\end{array}$ & $\begin{array}{l}\text { Diskussion in Kleingrup- } \\
\text { pen, Malen eines Plakats } \\
\text { und Vorstellung im } \\
\text { Plenum }\end{array}$ & $\begin{array}{l}\text { Bedürfnis- } \\
\text { pyramide nach } \\
\text { Schmidtchen } \\
\text { Plakate, Stifte, evtl. } \\
\text { Zeitungen für } \\
\text { Collagen }\end{array}$ \\
\hline $15^{\prime}$ & $\begin{array}{l}\text { Was passiert, wenn diese } \\
\text { Bedürfnisse nicht befriedigt } \\
\text { werden? } \\
\text { Was könnten Anzeichen } \\
\text { dafür sein? }\end{array}$ & $\begin{array}{l}\text { Erarbeitung im Plenum, } \\
\text { Ergänzungen durch die } \\
\text { Seminarleitung }\end{array}$ & $\begin{array}{l}\text { Arbeitsblatt aus } \\
\text { Anhang A4 }\end{array}$ \\
\hline
\end{tabular}

Tab. 3

\section{Sexuelle Entwicklung}

Ziele für die thematische Einbindung der sexuellen Entwicklung:

$>\quad$ TN haben Kenntnisse über die altersgemäße Entwicklung von Kindern und Jugendlichen, inkl. der sexuellen Entwicklung.

$>$ Sie können altersgemäßes Handeln erkennen und Abweichungen einschätzen. 


\begin{tabular}{|c|c|c|c|}
\hline Zeit & Inhalt & Methode & Material \\
\hline $45^{\prime}$ & $\begin{array}{l}\text { Altersgemäße Entwicklung } \\
\text { von Kindern und Jugend- } \\
\text { lichen }\end{array}$ & $\begin{array}{l}\text { In Kleingruppen: Jede Klein- } \\
\text { gruppe bekommt eine } \\
\text { Altersspanne zugewiesen } \\
\text { und trägt die wichtigsten } \\
\text { Punkte auf einem Plakat } \\
\text { zusammen, was im jewei- } \\
\text { ligen Altersabschnitt rele- } \\
\text { vant ist. (Unterschiede von } \\
\text { Jungen und Mädchen be- } \\
\text { achten, ohne zu stigma- } \\
\text { tisieren!) } \\
\text { Im Plenum: Vorstellung der } \\
\text { Gruppenergebnisse, evtl. } \\
\text { Ergänzung und Diskussion }\end{array}$ & $\begin{array}{l}\text { Plakate, Stifte } \\
\text { Für jede Alters- } \\
\text { gruppe Zusam- } \\
\text { menfassung der } \\
\text { entwicklungs- } \\
\text { psychologischen } \\
\text { Erkenntnisse }\end{array}$ \\
\hline $15^{\prime}$ & $\begin{array}{l}\text { Die sexuelle Entwicklung } \\
\text { des Menschen } \\
\text { Ziel: Die sexuelle Entwicklung } \\
\text { des Menschen beginnt schon } \\
\text { im Mutterleib, Unterschei- } \\
\text { dung von kindlicher und } \\
\text { erwachsener Sexualität }\end{array}$ & $\begin{array}{l}\text { Zeitstrahl: TN legen die } \\
\text { Kärtchen auf den Zeit- } \\
\text { strahl, ggf. schreiben sie } \\
\text { eigene Begriffe auf die } \\
\text { leeren Karten } \\
\text { Diskussion und Auswer- } \\
\text { tung }\end{array}$ & $\begin{array}{l}\text { Zeitstrahl, } \\
\text { Kärtchen mit Begrif- } \\
\text { fen zur sexuellen } \\
\text { Entwicklung } \\
\text { (Anhang A11.7), } \\
\text { leere Kärtchen }\end{array}$ \\
\hline
\end{tabular}

\section{Lebenswelten von Kindern und Jugendlichen}

In der Auseinandersetzung mit den Lebenswelten von Kindern und Jugendlichen ist die Sensibilisierung von Gefährdungssituationen einzubinden. Dabei kann den TN folgendes Wissen vermittelt werden:

$>$ Gefährdungssituationen wahrnehmen

$>$ Welche Anhaltspunkte für eine Gefährdung gibt es?

$>$ aufmerksames Beobachten im vielfältigen Alltag von Kindern und Jugendlichen 


\begin{tabular}{|c|c|c|c|}
\hline Zeit & Inhalt & Methode & Material \\
\hline $15^{\prime}$ & $\begin{array}{l}\text { Warmin' up zum Thema } \\
\text { Vielfalt, um herauszustellen, } \\
\text { dass die Lebenswelten von } \\
\text { Kindern und Jugendlichen } \\
\text { vielfältig sind. }\end{array}$ & $\begin{array}{l}\text { Darin unterscheide ich } \\
\text { mich von euch (Anhang } \\
\text { A11.1) }\end{array}$ & $\begin{array}{l}\text { Für jeden eine } \\
\text { Blankokarte, Stifte }\end{array}$ \\
\hline $30^{\prime}$ & $\begin{array}{l}\begin{array}{l}\text { Die Lebenswelt von Kin- } \\
\text { dern und Jugendlichen }\end{array} \\
\text { Arbeit in Kleingruppen }\end{array}$ & \begin{tabular}{|l|} 
Das Haus in der Müller- \\
straße (Anhang A11.2) \\
Die TN bekommen den \\
Auftrag, die Bewohner des \\
neu gebauten Hauses zu be- \\
schreiben: Wie sie leben und \\
was sie denken. Es sollen \\
möglichst unterschiedliche \\
Menschen dort wohnen.
\end{tabular} & $\begin{array}{l}\text { Große Papierbögen, } \\
\text { Stifte, evtl. vorge- } \\
\text { gebene Wohnfor- } \\
\text { men und Personen }\end{array}$ \\
\hline $20^{\prime}$ & $\begin{array}{l}\text { Vorstellen der Häuser, } \\
\text { evtl. auch Straßenzüge }\end{array}$ & & \\
\hline $20^{\prime}$ & $\begin{array}{l}\text { Mögliche Gefährdungen } \\
\text { von Kindern und Jugend- } \\
\text { lichen }\end{array}$ & $\begin{array}{l}\text { Die Seminarleitung spricht } \\
\text { anhand der vorgestellten } \\
\text { Familien mögliche Gefähr- } \\
\text { dungen an. }\end{array}$ & $\begin{array}{l}\text { Auszug aus diesem } \\
\text { Buch }\end{array}$ \\
\hline
\end{tabular}

\section{Baustein rechtliche Grundlagen}

Dieser Baustein gilt als Erweiterung im Bereich des Aufbauseminars und kann gut an die entwicklungspsychologische Einheit angeschlossen werden. Das erspart eine weitere Einführung und knüpft an eventuelle Diskussionen an, die sich aus dem Seminarinhalt ergeben können.

Ziele sind:

$>$ Die Seminarteilnehmenden kennen den Schutzauftrag nach $\$ 8$ a SGB VIII.

$>$ Sie wissen, was der Tätigkeitsausschluss nach \$72a SGB VIII ist.

$>$ Sie wissen über den Datenschutz Bescheid.

$>$ Sie haben Kenntnis über die Regelungen im Verband. 
3. Baustein Strukturen der verbandlichen Jugendarbeit

\begin{tabular}{|c|c|c|c|}
\hline Zeit & Inhalt & Methode & Material \\
\hline 5' & $\begin{array}{l}\text { Einstieg: Unser Verhaltens- } \\
\text { kodex }\end{array}$ & \begin{tabular}{|l|} 
Information über den Ent- \\
stehungshintergrund sowie \\
die rechtliche Einordnung
\end{tabular} & \\
\hline $15^{\prime}$ & $\begin{array}{l}\text { Wo begegnen uns Grenz- } \\
\text { verletzungen im Alltag der } \\
\text { Gruppenarbeit? } \\
\text { Zahlen und Fakten } \\
\text { Was ist sexualisierte Gewalt? } \\
\text { Was ist Vernachlässigung? }\end{array}$ & $\begin{array}{l}\text { Information und Samm- } \\
\text { lung im Plenum }\end{array}$ & $\begin{array}{l}\text { Aktuelle Zahlen } \\
\text { aus der Kriminal- } \\
\text { statistik } \\
\text { Definition }\end{array}$ \\
\hline $45^{\prime}$ & $\begin{array}{l}\text { Der Schutzauftrag in der } \\
\text { Jugendarbeit: } \\
\text { - §8a SGB VIII - Ablauf } \\
\text { bei Verdacht einer Kin- } \\
\text { deswohlgefährdung } \\
\text { - Tätigkeitsausschluss } \\
\text { nach } \$ 72 \text { a SGB VIII - } \\
\text { Ablauf bei Verdacht auf } \\
\text { Grenzverletzung oder } \\
\text { sexualisierter Gewalt in- } \\
\text { nerhalb des Verbandes } \\
\text { - Dokumentation }\end{array}$ & $\begin{array}{l}\text { Vortrag durch die Semi- } \\
\text { narleitung }\end{array}$ & \begin{tabular}{|l|} 
Arbeitsblätter: \\
- Auszüge aus dem \\
Buch Signale von \\
Kindeswohlgefähr- \\
dung (Anhang A4) \\
- Straftaten die einen \\
Tätigkeitsaus- \\
schluss beschrei- \\
ben (Anhang A2) \\
- Dokumentation \\
(Anhang A6) \\
- Handlungsleitfa- \\
den des Verbandes
\end{tabular} \\
\hline $15^{\prime}$ & Rückfragen & & \\
\hline 10 ' & $\begin{array}{l}\text { Unterschreiben der Selbst- } \\
\text { verpflichtung des Ver- } \\
\text { haltenskodexes }\end{array}$ & & $\begin{array}{l}\text { Verhaltenskodex } \\
\text { des Verbandes }\end{array}$ \\
\hline
\end{tabular}

Tab. 6

Das Unterschreiben der Selbstverpflichtungserklärung des Verhaltenskodexes könnte auch als gemeinsamer, feierlicher Akt am Ende der Seminarwoche stehen und damit den Willen unterstreichen, das eigene Engagement im Sinne der Regelungen zu gestalten.

\section{Baustein Strukturen der verbandlichen Jugendarbeit}

Im Baustein der JuLeiCa-Schulung, in dem es um die Struktur der verbandlichen Jugendarbeit geht, kann der Schutzauftrag in der 
Form eingebaut werden, dass die Ansprechpartner speziell für den Themenbereich Schutzauftrag genannt werden und auf den Handlungsleitfaden bzw. den Verhaltenskodex mit Selbstverpflichtung und die Grundsätze der verbandlichen Jugendarbeit hingewiesen wird.

Auch hier wäre die Möglichkeit der Unterzeichnung der Selbstverpflichtung einzubauen.

\section{Baustein Vertiefung}

Je nach Intensität kann dieser Baustein zeitlich variieren. Inklusive Pausen sollten für das vorgestellte Modul mindestens vier, eher fünf Zeitstunden eingeplant werden. Dieses Seminar ist für ehrenamtlich Tätige, die sich vertiefend mit dem Thema auseinandersetzten wollen und Kompetenzen für ihre Aufgaben, zum Beispiel als Freizeitleiter, erweitern möchten, geeignet. Einzelne Bausteine können entnommen werden, um zum Beispiel eine Seminarreihe zu machen.

Ziele für diese Seminareinheit sind:

$>$ Auseinandersetzung mit dem Schutzauftrag in der Jugendarbeit

$>$ Ursachen für Gefährdungen kennen

$>$ eigene Betroffenheit reflektieren 


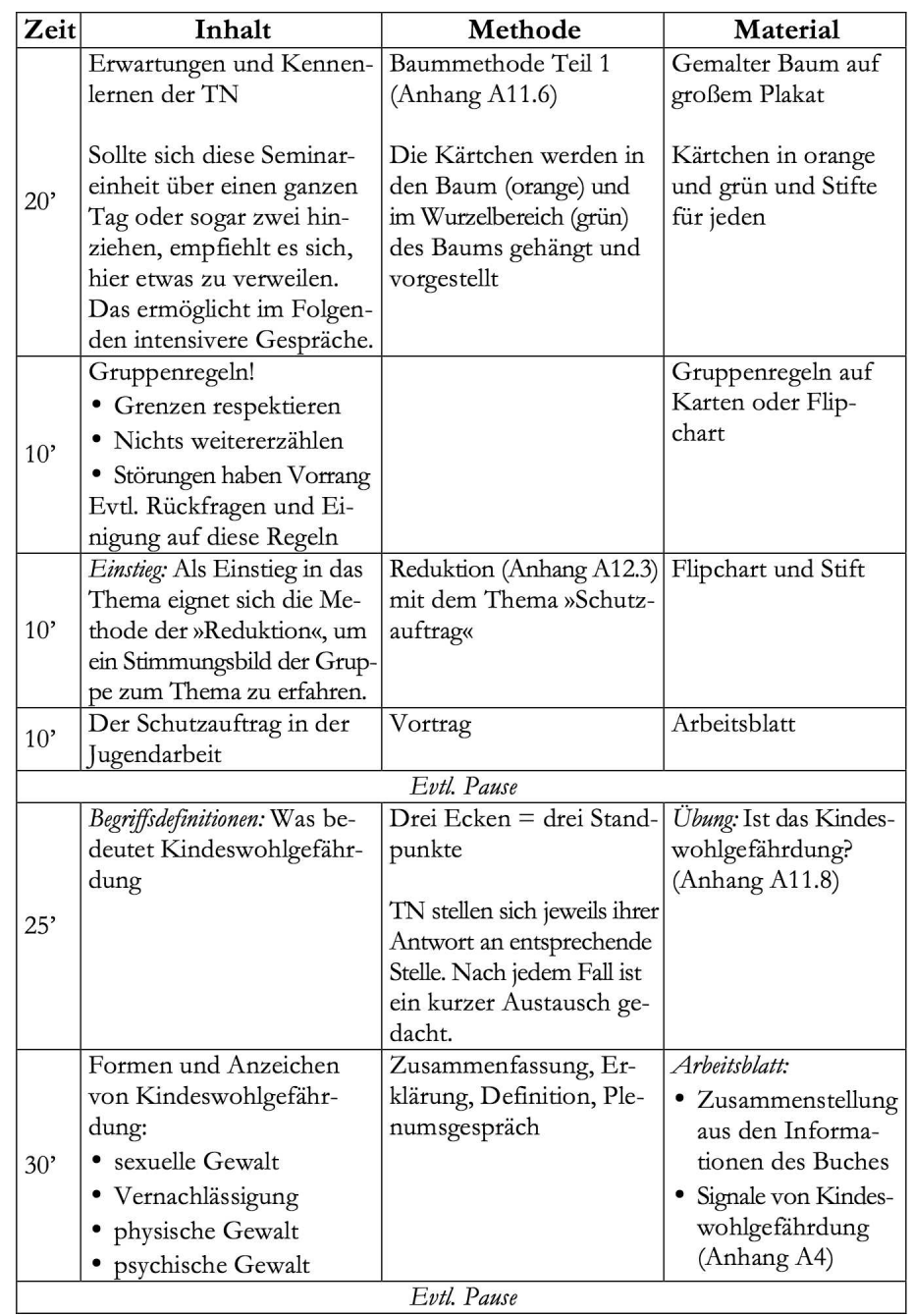




\begin{tabular}{|c|c|c|c|}
\hline Zeit & Inhalt & Methode & Material \\
\hline $10^{\prime}$ & $\begin{array}{l}\text { Als Mitarbeiter_in } \\
\text { verdächtigt werden }\end{array}$ & $\begin{array}{l}\text { „ topp«-Übung: TN teilen sich } \\
\text { in zwei Gruppen, die sich } \\
\text { gegenüber aufstellen (weiteres } \\
\text { Vorgehen, siehe Anhang) }\end{array}$ & $\begin{array}{l}\text { Übung: »Stopp« } \\
\text { (Anhang A11.5) }\end{array}$ \\
\hline $10^{\prime}$ & Auswertung & $\begin{array}{l}\text { Im Plenum werden die Emp- } \\
\text { findungen ausgetauscht. }\end{array}$ & \\
\hline $30^{\prime}$ & Konkretisierung & $\begin{array}{l}\text { Gespräch in Kleingruppen: } \\
\text { mittels kurzen Situationsbe- } \\
\text { schreibungen sollen TN da- } \\
\text { rüber ins Gespräch kommen, } \\
\text { was für sie harmlos, tolerabel } \\
\text { oder inakzeptabel ist. } \\
\text { Ziel: Jeder Mensch hat eine ei- } \\
\text { gene Empfindung von dem, } \\
\text { was richtig und falsch ist. Daher } \\
\text { ist es notwendig, bestimmte } \\
\text { Verhaltensregeln aufzustellen. }\end{array}$ & $\begin{array}{l}\text { Situationen zum The- } \\
\text { ma Nähe und Distanz } \\
\text { (Beispiele gibt es in den } \\
\text { Hand- und Methoden- } \\
\text { büchern, siehe An- } \\
\text { hang A10) oder eige- } \\
\text { ne Zusammenstellung }\end{array}$ \\
\hline \multicolumn{4}{|c|}{ Evtl. Pause } \\
\hline $20^{\prime}$ & $\begin{array}{l}\text { Vorgehen bei Verdacht } \\
\text { auf Kindeswohlge- } \\
\text { fährdung }\end{array}$ & $\begin{array}{l}\text { Anhand konkreter Fälle den } \\
\text { Ablauf erarbeiten und beschrei- } \\
\text { ben (evtl. kann hier noch eine } \\
\text { Kleingruppenarbeit vorgeschal- } \\
\text { tet werden): Regeln im Ver- } \\
\text { band vermitteln, verantwort- } \\
\text { liche Personen benennen, } \\
\text { Unterstützungsmöglichkeiten }\end{array}$ & $\begin{array}{l}\text { Fälle von Kindeswohl- } \\
\text { gefährdung entspre- } \\
\text { chend dem Aufgaben- } \\
\text { feld der Ehrenamt- } \\
\text { lichen (Beispiele für } \\
\text { Handlungsleitfaden } \\
\text { des Verbandes, siehe } \\
\text { Anhang A11.8) }\end{array}$ \\
\hline $15^{\prime}$ & $\begin{array}{l}\text { Selbstverpflichtung } \\
\text { zum Verhaltenskodex }\end{array}$ & $\begin{array}{l}\text { Als Verhaltenskodex kennen } \\
\text { und unterschreiben }\end{array}$ & $\begin{array}{l}\text { Verbandseigener } \\
\text { Verhaltenskodex }\end{array}$ \\
\hline $30^{\prime}$ & $\begin{array}{l}\text { Auswertung/Feed- } \\
\text { back/Eigenreflexion }\end{array}$ & Baummethode Teil 2 & $\begin{array}{l}\text { Baum vom Beginn des } \\
\text { Seminars, Körbchen, } \\
\text { Kärtchen in weiß, rot } \\
\text { und gelb, Stifte }\end{array}$ \\
\hline
\end{tabular}

\section{5. Überlegungen für Mitarbeiter_innenabende/ -kreise}

Für Mitarbeiter_innenkreise und -abende eignen sich kurze Einführungen oder Fallbeispiele, die zur Diskussion anregen. Hier eignen sich Fragestellungen, die die Lebenswelt der anwesenden Jugendlichen betreffen. Die eigene Gefährdung wird in der Regel nicht 
so gesehen, wie sie Erwachsene wahrnehmen würden. Die Grenzen von Gefährdung sind je nach Peergroup unterschiedlich (Beschaffungsprostitution, EMO-Bewegung, Ritzen als Trend ...) und der Graubereich muss immer wieder definiert werden. Mit der Definition von diesen Situationen ist ein Einstieg in eine Auseinandersetzung und damit Sensibilisierung gegeben. Dabei können folgende Fragen helfen:

$>$ Was ist $\gg$ normal $\ll$ ?

$>$ Was kann ich nicht einschätzen?

$>$ Was ist für mich Kindeswohlgefährdung?

Auch die angrenzenden Themen zum Schutz des Wohls von Kindern und speziell auch von Jugendlichen sind bei solchen Abenden interessant:

$>\quad$ Nutzung der neuen Medien

$>$ Mobbing

$>$ Sexualität allgemein

$>$ Gewalt

Jugendliche Mitarbeitende können immer auch selbst von einer Kindeswohlgefährdung betroffen sein. Gegebenenfalls ist die Gefährdung eine andere und eventuell nicht so massiv wie bei jüngeren Kindern. Dennoch brauchen auch sie Unterstützung. Aufgabe der Jugendarbeit kann hier zum Beispiel die Unterstützung der Jugendlichen bei Autonomiekonflikten mit den Eltern sein.

\section{Schulungsmodul für die Freizeitvorbereitung}

Wenn die Umsetzung des Schutzauftrages in der verbandlichen Jugendarbeit ernst genommen wird, muss gerade im Bereich der Freizeiten dieses Thema nochmals intensiver betrachtet werden. Auch »alte Hasen «, die diesem Thema durch jahrelange Erfahrung im Freizeitenbereich schon begegnet sind, sollten sich in einem methodischen Rahmen fachlich damit auseinandersetzen.

In den Schulungen könnte praktisch auf Erfahrungen in Freizeiten aufgebaut werden. Konkrete Themen könnten die Gruppendy- 
namiken auf Freizeiten mit zum Teil grenzüberschreitenden Formen sein, die aus $\gg S p a ß \ll$ beginnen und dann in den Bereich des Kinderschutzes fallen (siehe unten stehender Vorschlag). Hier geht es um Situationen, die aus dem Kreis der Teilnehmenden heraus auf Freizeiten entstehen und eine klare Position der Freizeitleitung benötigt. Diese sollte in Seminaren herausgearbeitet und reflektiert werden. Ein weiterer Baustein müsste sich mit den Fällen von Kindeswohlgefährdung befassen, die durch das gewachsene Vertrauen und damit verbundene Gespräche auf Freizeiten auftauchen können und nicht die Situation auf der Freizeit betreffen, sondern die Lebenswelt des betreffenden Teilnehmenden der Freizeit. Hier benötigen Freizeitmitarbeitende klare Informationen und Kenntnisse über die Gesetzeslage und die Regelungen im Verband. Das Thema Nähe-Distanz ist wichtig für den Bereich der Freizeiten und Ferienwaldheimarbeit. Dabei muss das unterschiedliche Wahrnehmen von eigenem und fremdem Verhalten thematisiert und reflektiert werden. Hier kann schon Beschriebenes für den Freizeitenbereich übernommen werden (siehe »Stopp«-Übung im Baustein Vertiefung und weiterführende Inhalte). Eine sorgfältige Auswertung und Reflexion ist obligatorisch.

Ziele für ein Seminar zu grenzverletzendem Verhalten von Teilnehmenden auf Freizeiten sind:

$>$ TN setzen sich mit Situationen auseinander, in denen es um grenzverletzendes Verhalten unter den Teilnehmenden auf einer Freizeit gekommen ist

$>$ Entstehungsbedingungen werden symbolisch dargestellt

$>\quad$ eine eigene Position zu Gewalt reflektiert

$>$ mögliche Handlungsschritte erarbeitet 


\begin{tabular}{|c|c|c|c|}
\hline Zeit & Inhalt & Methode & Material \\
\hline & $\begin{array}{l}\text { Einstieg: Ziele von Freizei- } \\
\text { ten bezüglich der TN }\end{array}$ & $\begin{array}{l}\text { Zielscheibe (Anhang } \\
\text { A12.4), zum Thema Frei- } \\
\text { zeiten erst in Einzelarbeit, } \\
\text { dann Austausch in Klein- } \\
\text { gruppen, Vorstellung der } \\
\text { Gruppenzielscheibe im } \\
\text { Plenum }\end{array}$ & $\begin{array}{l}\text { Papier mit Zielschei- } \\
\text { ben (Vorlage: An- } \\
\text { hang A12.4) }\end{array}$ \\
\hline & $\begin{array}{l}\text { Sammlung von grenzüber- } \\
\text { schreitenden Situationen auf } \\
\text { Freizeiten (evtl. Situationen } \\
\text { bereithalten, z.B. Szenen ei- } \\
\text { nes Zeltlagers*) }\end{array}$ & & $\begin{array}{l}\text { Kärtchen oder Flip- } \\
\text { chart, evtl. Szenen } \\
\text { eines Zeltlagers ko- } \\
\text { pieren und auf ein- } \\
\text { zelnen Karten* }\end{array}$ \\
\hline & $\begin{array}{l}\text { Auseinandersetzung mit } \\
\text { Grenzüberschreitungen } \\
\text { innerhalb der Gruppe - } \\
\text { Erfahrungen }\end{array}$ & $\begin{array}{l}\text { Skulpturen von Situationen } \\
\text { in Kleingruppen stellen; } \\
\text { dann: Reihen stellen } \\
\text { - Was ist (könnte) davor } \\
\text { passiert (sein)? } \\
\text { - Was passiert danach? } \\
\text { - Wie wäre die Situation } \\
\text { angenehmer? }\end{array}$ & $\begin{array}{l}\text { Verschiedene Situ- } \\
\text { ationen von Frei- } \\
\text { zeiten auf Kärtchen } \\
\text { oder an die Tafel } \\
\text { schreiben }\end{array}$ \\
\hline & $\begin{array}{l}\text { Auswertung der Skulp- } \\
\text { turen }\end{array}$ & $\begin{array}{l}\text { TN berichten von ihren Er- } \\
\text { fahrungen in der Skulptur: } \\
\text { - Wie habe ich mich ge- } \\
\text { fühlt? } \\
\text { - Was hat sich mit der } \\
\text { Veränderung der Skulp- } \\
\text { tur in meiner Empfin- } \\
\text { dung verändert? }\end{array}$ & \\
\hline
\end{tabular}

(*) vgl. Evangelische Jugendarbeit in Bayern (2004), »Bei uns nicht!? «

Gemeinsam gegen sexuellen Missbrauch im Jugendverband (2. Aufl., S. 58f.).

\section{Tab. 8}




\section{Fazit}

Wenn sich verbandliche Jugendarbeit entscheidet, den Schutzauftrag nachhaltig zu integrieren, muss sie sich Prävention als Grundhaltung zu eigen machen. Allein die Umsetzung von Schlaglichtern reicht nicht aus, um das Selbstverständnis und die Haltung zu verändern. In den vergangenen Jahren rückte das Thema besonders in den Fokus und auch verbandliche Jugendarbeit beschäftigt sich zunehmend damit. Bei Jörg M. Fegert und Mechthild Wolff (2006, S. 309) ist zu lesen, dass das Thema Kindeswohlgefährdung im Verband verunsichert und es daher umso wichtiger sei, mit dieser Thematik offen und sensibel umzugehen. So ist das Thema Kindeswohlgefährdung und Schutz vor sexualisierter Gewalt als Selbstverständlichkeit in den Schulungen verbandlicher Jugendarbeit aufzunehmen und zu verinnerlichen. Das sollte meines Erachtens in Form von eigenen Seminarinhalten und als Querschnitt durch die angrenzenden Themen geschehen.

Auch wenn die Umsetzung des \$8a SGB VIII nicht alle Verbände der Kinder- und Jugendarbeit formal gesetzlich betrifft, haben sie dennoch den Anspruch, ein geschützter Raum für Kinder und Jugendliche zu sein. Verbandliche Jugendarbeit ist allein durch ihr Selbstverständnis ein wesentlicher Faktor in der Prävention und Stärkung von Selbstbewusstsein von Kindern und Jugendlichen. Daher kann sie sich nicht davor verschließen und sollte sich mit eigene Handlungsschritten und -möglichkeiten auseinandersetzen. Das ist für größere Verbände personell sicher einfacher umzusetzen, hat in der Kommunikation aber auch größere Hürden zu meistern. In Zusammenschlüssen wie Stadtjugend- 
ringen oder Kreisjugendringen, die besonders für kleinere und ehrenamtlich geleitete Verbände die JuLeiCa-Schulungen anbieten, kann für diese Verbände ein Forum geschaffen werden. Die Auseinandersetzung innerhalb des Verbandes ersetzt das jedoch nicht.

Soll der Schutz bei Kindeswohlgefährdung und vor sexualisierter Gewalt nicht nur ein zeitlich begrenztes Phänomen bleiben, muss er als Querschnitts- und Leitungsaufgabe im Verband gesehen und verankert werden. Dafür sind Vereinbarungen mit den öffentlichen Jugendhilfeträgern und Standards, wie sie der UBSMK festgeschrieben hat, hilfreich und nützlich. 2010 beschrieb Mechthild Wolff die Beobachtung, dass Verbände, die sich auf den Weg gemacht haben, das nicht durch Standards taten, sondern aus dem Bewusstsein heraus, Verantwortung zu übernehmen (z. B. der Bayerische Landesjugendring mit dem Modellprojekt PräTect). Die Veränderung institutioneller Kulturen benötigt Zeit, Personal und Geld und kann nicht allein durch Standards verordnet werden. Vielmehr lösen Verordnungen Ängste und Widerstände aus (vgl. Wolff, 2010, S. 539). Hier sind Entscheidungen über Ressourcen im Jugendverband notwendig. Das ist in Zeiten knapper werdender Mittel sicher nicht leicht. Dennoch kann meines Erachtens der Weg zu einer gelebten Kultur des Kinderschutzes und der Achtsamkeit gegenüber Grenzverletzungen in der verbandlichen Jugendarbeit nicht »nebenher « beschritten werden. 


\section{Literatur}

Alle, F. (2010). Kindeswohlgefährdung. Das Praxishandbuch. Freiburg: Lambertus-Verlag. Bayerisches Landesjugendamt (BLJA) (2012). Fachliche Empfehlungen (neu nach Bundeskinderschutzgesetz) Empfehlungen zur Umsetzung des Schutzauftrags nach §8a SGB VIII. http://www.blja.bayern.de/imperia/md/content/blvf/bayer landesjugendamt/fachliche_empfehlungen_8a_stand_10.07.2012_barrierefr ei.pdf (28.05.2016).

Bender, D. \& Lösel, F. (2005). Misshandlung von Kindern: Risikofaktoren und Schutzfaktoren. In G. Deegener \&W. Körner (Hrsg.), Kindesmisshandlung und Vernachlässigung. Ein Handbuch (S. 317-346). Göttingen: Hogrefe Verlag.

Bergmann, C. (2011). Abschlussbericht Runder Tisch Sexueller Kindesmissbrauch in Abhängigkeits- und Machtverhältnissen in privaten und öffentlichen Einrichtungen und im familiären Bereich. Bundesdruckerei.

Bericht der Bundesregierung (2015). Evaluation des Bundeskinderschutzgesetzes. http: //www.bmfsfj.de/RedaktionBMFSFJ/Abteilung5/Pdf-Anlagen/bericht-evaluat ion-bundeskinderschutzgesetz,property $=$ pdf, bereich $=$ bmfsfj,sprache $=$ de, $r w$ b=true.pdf (25.05.2016).

Bundesministerium für Familien Senioren, Frauen und Jugend (BMFSFJ) (2015). Pressemitteilung zur Evaluation des Bundeskinderschutzgesetzes. http://www.bmfsfj. de/BMFSFJ/kinder-und-jugend,did=222322.html (26.05.2016).

Conen, M. (2006). Arbeitshilfe für die Personalauswahl zur Vermeidung der Einstellung pädophiler Mitarbeiter In J. Fegert \& M. Wolff (Hrsg.), Sexueller Missbrauch durch Professionelle in Institutionen. Prävention und Intervention - ein Werkbuch (2., aktualis. Aufl.; S. 56-63). Weinheim/München: Beltz Juventa.

Deegener, G. (Hrsg.). (2005). Kindesmisshandlung und Vernachlässigung. Ein Handbuch. Göttingen: Hogrefe Verlag.

Deinet, U. (2006). Expertise: Schutzauftrag bei Kindeswohlgefährdung - Kinder- und Jugendarbeit. Sozialextra, 10, 28-31.

Deutscher Bundesjugendring (DBJR) (2015). Evaluation zum Bundeskinderschutzgesetz. https://www.dbjr.de/dbjr-info/artikel/detail/evaluationsbericht-zum -bundeskinderschutzgesetz.html (12.05.2016). 
Deutscher Bundesjugendring (DBJR) (2008). Stellungnahme zum Referentenentwurf eines Bundesgesetzes zur Verbesserung des Kinderschutzes. https://www.dbjr. de/fileadmin/user_upload/pdf-dateien/Stellungnahmen/Stellungnahme_ BKiSchG.pdf (28.05.2016).

Deutscher Bundesjugendring (DBJR) (2010). Hintergrundpapier des Deutschen Bundesjugendrings zum Stand der Entwicklung und Umsetzung umfassender Präventionsansätze in der Jugend(verbands)arbeit. https://www.dbjr.de/fileadmin/ user_upload/pdf-dateien/Hintergrundpapier/Hintergrundpapier_ Praevention.pdf (21.05.2016).

Deutscher Bundesjugendring (DBJR) (2016). Stellungnahme zur Evaluation des Bundeskinderschutzgesetzes. https://www.dbjr.de/fileadmin/user_upload/pdf -dateien/Stellungnahmen/DBJR-Stellungnahme-BKiSchG.pdf

Deutscher Bundesjugendring (DBJR) (2016). Zum Bericht der Bundesregierung: Evaluation des Bundeskinderschutzgesetzes. Stellungnahme. https://www.dbjr.de/ fileadmin/user_upload/pdf-dateien/Stellungnahmen/DBJR-Stellungnahme -BKiSchG.pdf (12.05.2016).

Deutscher Verein für öffentliche und private Fürsorge e.V. (2012). Empfehlung des Deutschen Vereins zu Führungszeugnissen bei Neben- und Ehrenamtlichen in der Kinder- und Jugendhilfe. https://www.deutscher-verein.de/de/empfehlungen -stellungnahmen-2012-empfehlungen-des-deutschen-vereins-zu -fuehrungszeugnissen-bei-neben-und-ehrenamtlichen-in-der-kinder-und -jugendhilfe-72-a-abs-3-und-abs-4-sgb-viii-1-1528,311,1000.html (20.05.2016).

Eichholz, R. (2009). Der Vorrang des Kindeswohls nach Art. 3 UN-Kinderrechtskonvention. Konsequenz für die bundesdeutsche Praxis. IzKK-Nachrichten, 9(1), $12-15$.

Enders, U. (2012). Grenzen achten: Schutz vor sexuellem Missbrauch in Institutionen. Ein Handbuch für die Praxis. Köln: KiWi-Taschenbuch.

Evangelische Jugendarbeit in Bayern (EJB) (2004). Bei uns nicht! Gemeinsam gegen sexuellen Missbrauch im Jugendverband (2. Aufl.). Nürnberg.

Fegert, J., Hoffmann, U., König, E., Niehus, J \& Liebhardt, H. (2015). Sexueller Missbrauch von Kindern und Jugendlichen. Ein Handbuch zur Prävention und Intervention für Fachkräfte im medizinischen, psychotherapeutischen und pädagogischen Bereich. Berlin/Heidelberg: Springer Verlag.

Fegert, J. \& Wolff, M. (2015). Kompendium »Sexueller Missbrauch in Institutionen«. Entstehungsbedingungen, Prävention und Intervention. Weinheim \& Basel: Beltz/Juventa.

Fegert, J. \& Wolff, M. (2006). Sexueller Missbrauch durch Professionelle in Institutionen. Prävention und Intervention. Ein Werkbuch. Weinheim \& München: Juventa.

Hoyer, T. (2015). Sozialgeschichte der Erziehung. Darmstadt: WBG.

Institut für soziale Arbeit e.V. (ISA) (2006). Der Schutzauftrag bei Kindeswohlgefährdung - Arbeitshilfe zur Kooperation zwischen Jugendamt und Trägern der freien Kinder- und Jugendhilfe. Münster.

Jans, K.-W., Happe, G, Saurbier, H. \& Maas, U. (2015). Kinder- und Jugendhilferecht, Band 2, Loseblatt, 52. Ergänzungslieferung 2015. Stuttgart: Kohlhammer.

Jordan, E. \& Bathke, S. (2007). Die Chancen, Sicherheit zu gewinnen ... Arbeitsgruppe 2: Zusammenarbeit in öffentlichen und freien Trägern bei der Wahr- 
nehmung des Schutzauftrages - unter Bezugnahme auf Vorschläge und Anregungen des ISA (Arbeitshilfe). In Verein für Kommunalwissenschaften e.V. (Hrsg.), Kinderschutz gemeinsam gestalten: §8a SGB VIII - Schutzauftrag der Kinder- und Jugendhilfe. Dokumentation der Fachtagung am 22. und 23. Juni 2006 in Berlin (S. 95-106). Berlin: Verein für Kommunalwissenschaften e. V.

Jung, H-P. Sozialgesetzbuch (SGB) für die Praxis. Achtes Buch (VIII). Kinder- und Jugendhilfe. Kommentar begründet von Prof. Dr. Kurt Jahn. Freiburg, München \& Würzburg: Haufe Mediengruppe.

Key, Ellen (1902). Das Jahrhundert des Kindes. Studien. Autorisierte Übertragung von Francis Maro. Berlin: S. Fischer Verlag.

Kindler, H. (2007). Kinderschutz in Deutschland stärken. Analyse des nationalen und internationalen Forschungsstandes zu Kindeswohlgefährdung und die Notwendigkeit eines nationalen Forschungsplanes zur Unterstützung der Praxis. Eine Expertise im Auftrag des Informationszentrums Kindesmisshandlung/Kindesvernachlässigung. München: DJl-Arbeitspapier.

Kindler, H. \& Reich, W. (2007). Die Chancen, Sicherheit zu gewinnen ... Arbeitsgruppe 1: Einschätzung von Gefährdungsrisiken (Instrumente und Hilfen) am Beispiel der weiterentwickelten Version des Stuttgarter Kinderschutzbogens. In Verein für Kommunalwissenschaften e.V. (Hrsg.), Kinderschutz gemeinsam gestalten: §8a SGB VIII - Schutzauftrag der Kinder- und Jugendhilfe. Dokumentation der Fachtagung am 22. und 23. Juni 2006 in Berlin (S. 63-94). Berlin: Verein für Kommunalwissenschaften e.V.

Kindler, H., Lilllig, S. Blüml, H., Meysen, T. \& Werner, A. (Hrsg.). (2006). Handbuch Kindeswohlgefährdung nach \$1666 BGB und Allgemeiner sozialer Dienst (ASD). http:// db.dji.de/asd/ASD_Handbuch_Gesamt.pdf (15.01.2015).

Kindler; H. \& Lillig, S. (2005). Früherkennung von Familien mit erhöhten Misshandlungs- oder Vernachlässigungsrisiken. IKK-Nachrichten, 5(1-2), 10-13.

Kommunalverband für Jugend und Soziales Baden-Württemberg (KVJS) (2014). Zusammenfassende arbeitsfeldspezifische Hinweise zu Vereinbarungen zum Schutzauftrag der Jugendhilfe gem. §§8a Abs. 4 und 72a SGB VIII mit Trägern von Einrichtungen und Diensten in den Arbeitsfeldern Jugendarbeit, Jugendsozialarbeit, Erzieherischer Kinder- und Jugendschutz nach \$\$11-15 SGB VIII. http:// www.kvjs.de/fileadmin/dateien/jugend/kinderschutz/schutzauftrag_ materialpool/Hinweise_Jugendarbeit_mit_Aenderungen.pdf (03.06.2016).

Kommunalverband für Jugend und Soziales Baden-Württemberg (KVJS) (2009). KVJS-Ratgeber, Arbeitshilfe zur Umsetzung des Schutzauftrags bei Kindeswohlgefährdung und zur Prüfung der persönlichen Eignung von Fachkräften. Stuttgart.

Krieger, W., Lang, A., Messmer, S. \& Osthoff, R. (2007). Kindesmisshandlung, Vernachlässigung und sexueller Missbrauch im Aufgabengebiet der öffentlichen Träger der Jugendhilfe. Eine Einführung. Stuttgart: Ibidemverlag.

Kunkel, P.-C. (2015). Jugendhilferecht (8. Aufl.). Baden-Baden: Nomos Verlag.

Kunkel, P.-C. (2006). Schutzauftrag bei Kindeswohlgefährdung (\$8a SGB VIII) - rechtliche und psychologische Dimension. Kehler Diskussionspapier Nr. 2006-3.

Landesjugendring Baden-Württemberg (LJRBW) (2009). Standards der Jugendleiterlnnen-Ausbildung. Selbstverpflichtung der Jugendverbände und -ringe in Baden Württemberg. Stuttgart: Eigenverlag. 
Maywald, J.(2014). Kindergarten Heute spezial: Kindeswohlgefährdung - erkennen, einschätzen, handeln (2., völlig überarb. Neuaufl.). Freiburg: Herder Verlag.

Meier, F. (2006). Mit Kind und Kegel. Kindheit und Familie im Wandel der Geschichte. Ostfildern: Jan Thorbecke Verlag.

Mertes, K. (2013). Verlorenes Vertrauen. Katholisch sein in der Krise. Freiburg: Herder Verlag.

Meysen, Th. (2007). Rechtliche Vorgaben zur Kommunikation bei interdisziplinärer Kooperation. In U. Ziegenhain \& J. Fegert (Hrsg.) (2008), Kindeswohlgefährdung und Vernachlässigung (S. 30-36). München: Ernst Reinhardt Verlag.

Moggi, F. (2005). Folgen von Kindesmisshandlung: Ein Überblick. In G. Deegener (Hrsg.), Kindesmisshandlung und Vernachlässigung. Ein Handbuch (S. 94-103). Göttingen: Hogrefe Verlag.

Münder, J. Meysen Th. \& Trenczek, Th. (2012). Frankfurter Kommentar SGB VIII: Kinder und Jugendhilfe (7. Aufl.). Baden-Baden: Nomos Verlag.

Münder, J., Mutke, B. \& Schone, R. (2000). Kindeswohl zwischen Jugendhilfe und Justiz. Münster: Votum Verlag.

Salgo, L. (2007). §8a SGB VIII - Anmerkungen und Überlegungen zur Vorgeschichte und den Konsequenzen der Gesetzesänderung. In U. Ziegenhain \& J. Fegert (Hrsg.) (2008), Kindeswohlgefährdung und Vernachlässigung (S. 9-29). München: Ernst Reinhardt Verlag.

Schnapka, M \& Stroppel, M. (2006). Wenn Erzieher Täter werden ... In J. Fegert \& M. Wolff (Hrsg.), Sexueller Missbrauch durch Professionelle in Institutionen. Prävention und Intervention. Ein Werkbuch (S. 172-175). Weinheim \& München: Juventa.

Steinbach, B. (2015). Prävention von sexueller Gewalt im Ehrenamtssektor. In J. Fegert \& M. Wolff (Hrsg.), Kompendium »Sexueller Missbrauch in Institutionen«. Entstehungsbedingungen, Prävention und Intervention. (S. 186-196). Weinheim \& Basel: Beltz/Juventa.

Trenczek, Th. (2008). Inobhutnahme. Krisenintervention und Schutzgewährung durch die Jugendhilfe. $\$ \$ 8 a, 42$ SGB VIII. Stuttgart: Richard Boorberg Verlag.

Tschörpe-Scheffler, S. (2009). Fünf Säulen der Erziehung. Mainz: Grünewald-Verlag.

UBSKM. Was ist sexueller Missbrauch? https://beauftragter-missbrauch.de/praeventio n/was-ist-sexueller-missbrauch/ (05.01.2015).

UNICEF (2015). Geschichte der Kinderrechte. http://www.unicef.ch/sites/default/files/ attachements/unicef_fs_geschichte_der_kinderrechte_2015_0.pdf(23.05.2016).

Vereinte Nationen (1959). Erklärung der Rechte des Kindes. http://www.kinderrechtsko nvention.info/erklaerung-der-rechte-des-kindes-vom-20-november-1959-33 47/ (25.05.2016).

Voigts, G. (2006). Arbeitsgruppe 5: Die Chancen der Zusammenarbeit bei der Wahrnehmung des Schutzauftrages nach §8a SGB VIII von Jugendamt und Jugendarbeit. In Verein für Kommunalwissenschaften e. V. (Hrsg.), Kinderschutz gemeinsam gestalten: §8a SGB VIII - Schutzauftrag der Kinder- und Jugendhilfe. Dokumentation der Fachtagung am 22. und 23. Juni 2006 in Berlin (S. 175-182). Berlin: Verein für Kommunalwissenschaften e.V.

Voigts, G. (2005). Der Schutzauftrag bei Kindeswohlgefährdung im Kinder- und Jugendhilfeerweiterungsgesetz (KICK) und deren Bedeutung für die Angebote der verbandlichen Kinder- und Jugendarbeit. AEJ INFORMATION, 4, 48-52. 
Wiesner, R. (2015). SGB VIII Kinder- und Jugendhilfe. Kommentar. München: C. H. Beck.

Wiesner, R. (2006). Präzisierung und Qualifizierung der Aufgaben der Jugendhilfe nach §8a SGB VIII. Verein für Kommunalwissenschaften e.V. (Hrsg.), Kinderschutz gemeinsam gestalten: §8a SGB VIII - Schutzauftrag der Kinder- und Jugendhilfe. Dokumentation der Fachtagung am 22. und 23. Juni 2006 in Berlin (S. 53-62). Berlin: Verein für Kommunalwissenschaften e.V.

Wipplinger, R. \& Amman, G. (2005). Sexueller Missbrauch. Überblick zu Forschung, Beratung und Therapie. Ein Handbuch. Tübingen: dgvt-Verlag.

Wolff, M. (2010). Mindeststandards für den verbindlichen Schutz von Kindern und Jugendlichen in Institutionen - Ein Kommentar. Deutsches Insititut für Jugendhilfe und Familienrecht e. V., Das Jugendamt - Zeitschrift für Jugendhilfe und Familienrecht. 538-540.

Zartbitter e.V. Enders, Kossatz, Y, Kelkel, M. \& Eberhardt, B. (2010). Zur Differenzierung zwischen Grenzverletzungen, Übergriffen und strafrechtlich relevanten Formen der Gewalt im pädagogischen Alltag. http://www.praevention-bild ung.dbk.de/fileadmin/redaktion/praevention/microsite/Downloads/Zartbitt er_GrenzuebergriffeStraftaten.pdf (25.05.2016).

Ziegenhain, U. \& Fegert, J. (2008). Kindeswohlgefährdung und Vernachlässigung. München: Ernst Reinhardt Verlag. 


\section{Anhang}

\section{A1 Gesetzliche Grundlagen - Auszüge}

\section{A1.1 Sozialgesetzbuch achtes Buch (SGB VIII)}

$\$ 1$ Recht auf Erziehung, Elternverantwortung, Jugendhilfe

(1) Jeder junge Mensch hat ein Recht auf Förderung seiner Entwicklung und auf Erziehung zu einer eigenverantwortlichen und gemeinschaftsfähigen Persönlichkeit.

(2) Pflege und Erziehung der Kinder sind das natürliche Recht der Eltern und die zuvörderst ihnen obliegende Pflicht. Über ihre Betätigung wacht die staatliche Gemeinschaft.

(3) Jugendhilfe soll zur Verwirklichung des Rechts nach Absatz 1 insbesondere

1. junge Menschen in ihrer individuellen und sozialen Entwicklung fördern und dazu beitragen, Benachteiligungen zu vermeiden oder abzubauen,

2. Eltern und andere Erziehungsberechtigte bei der Erziehung beraten und unterstützen,

3. Kinder und Jugendliche vor Gefahren für ihr Wohl schützen,

4. dazu beitragen, positive Lebensbedingungen für junge Menschen und ihre Familien sowie eine kinder- und familienfreundliche Umwelt zu erhalten oder zu schaffen. 


\section{I8 SGB VIII Beteiligung von Kindern und Jugendlichen}

(1) Kinder und Jugendliche sind entsprechend ihrem Entwicklungsstand an allen sie betreffenden Entscheidungen der öffentlichen Jugendhilfe zu beteiligen. Sie sind in geeigneter Weise auf ihre Rechte im Verwaltungsverfahren sowie im Verfahren vor dem Familiengericht und dem Verwaltungsgericht hinzuweisen.

(2) Kinder und Jugendliche haben das Recht, sich in allen Angelegenheiten der Erziehung und Entwicklung an das Jugendamt zu wenden.

(3) Kinder und Jugendliche haben Anspruch auf Beratung ohne Kenntnis des Personensorgeberechtigten, wenn die Beratung auf Grund einer Not- und Konfliktlage erforderlich ist und solange durch die Mitteilung an den Personensorgeberechtigten der Beratungszweck vereitelt würde. $\$ 36$ des Ersten Buches bleibt unberührt.

\section{8a Schutzauftrag bei Kindeswoblgefährdung}

(1) Werden dem Jugendamt gewichtige Anhaltspunkte für die Gefährdung des Wohls eines Kindes oder Jugendlichen bekannt, so hat es das Gefährdungsrisiko im Zusammenwirken mehrerer Fachkräfte einzuschätzen. Soweit der wirksame Schutz dieses Kindes oder dieses Jugendlichen nicht in Frage gestellt wird, hat das Jugendamt die Erziehungsberechtigten sowie das Kind oder den Jugendlichen in die Gefährdungseinschätzung einzubeziehen und, sofern dies nach fachlicher Einschätzung erforderlich ist, sich dabei einen unmittelbaren Eindruck von dem Kind und von seiner persönlichen Umgebung zu verschaffen. Hält das Jugendamt zur Abwendung der Gefährdung die Gewährung von Hilfen für geeignet und notwendig, so hat es diese den Erziehungsberechtigten anzubieten.

(2) Hält das Jugendamt das Tätigwerden des Familiengerichts für erforderlich, so hat es das Gericht anzurufen; dies gilt auch, wenn die Erziehungsberechtigten nicht bereit oder in der Lage sind, bei der Abschätzung des Gefährdungsrisikos mitzuwirken. Besteht eine dringende Gefahr und kann die Entscheidung des Gerichts nicht abgewartet werden, so ist das Jugendamt verpflichtet, das Kind oder den Jugendlichen in Obhut zu nehmen. 
(3) Soweit zur Abwendung der Gefährdung das Tätigwerden anderer Leistungsträger, der Einrichtungen der Gesundheitshilfe oder der Polizei notwendig ist, hat das Jugendamt auf die Inanspruchnahme durch die Erziehungsberechtigten hinzuwirken. Ist ein sofortiges Tätigwerden erforderlich und wirken die Personensorgeberechtigten oder die Erziehungsberechtigten nicht mit, so schaltet das Jugendamt die anderen zur Abwendung der Gefährdung zuständigen Stellen selbst ein.

(4) In Vereinbarungen mit den Trägern von Einrichtungen und Diensten, die Leistungen nach diesem Buch erbringen, ist sicherzustellen, dass

1. deren Fachkräfte bei Bekanntwerden gewichtiger Anhaltspunkte für die Gefährdung eines von ihnen betreuten Kindes oder Jugendlichen eine Gefährdungseinschätzungvornehmen,

2. bei der Gefährdungseinschätzung eine insoweit erfahrene Fachkraft beratend hinzugezogen wird sowie

3. die Erziehungsberechtigten sowie das Kind oder der Jugendliche in die Gefährdungseinschätzung einbezogen werden, soweit hierdurch der wirksame Schutz des Kindes oder Jugendlichen nicht in Frage gestellt wird.

In die Vereinbarung ist neben den Kriterien für die Qualifikation der beratend hinzuzuziehenden insoweit erfahrenen Fachkraft insbesondere die Verpflichtung aufzunehmen, dass die Fachkräfte der Träger bei den Erziehungsberechtigten auf die Inanspruchnahme von Hilfen hinwirken, wenn sie diese für erforderlich halten, und das Jugendamt informieren, falls die Gefährdung nicht anders abgewendet werden kann.

(5) Werden einem örtlichen Träger gewichtige Anhaltspunkte für die Gefährdung des Wohls eines Kindes oder eines Jugendlichen bekannt, so sind dem für die Gewährung von Leistungen zuständigen örtlichen Träger die Daten mitzuteilen, deren Kenntnis zur Wahrnehmung des Schutzauftrags bei Kindeswohlgefährdung nach $\$ 8$ a erforderlich ist. Die Mitteilung soll im Rahmen eines Gespräches zwischen den Fachkräften der beiden örtlichen Träger erfolgen, an dem die Personensorgeberechtigten sowie das Kind oder der Jugendliche beteiligt werden sollen, soweit hierdurch der wirksame Schutz des Kindes oder des Jugendlichen nicht in Frage gestellt wird. 
58b SGB VIII Fachliche Beratung und Begleitung zum Schutz von Kindern und Jugendlichen

(1) Personen, die beruflich in Kontakt mit Kindern oder Jugendlichen stehen, haben bei der Einschätzung einer Kindeswohlgefährdung im Einzelfall gegenüber dem örtlichen Träger der Jugendhilfe Anspruch auf Beratung durch eine insoweit erfahrene Fachkraft.

(2) Träger von Einrichtungen, in denen sich Kinder oder Jugendliche ganztägig oder für einen Teil des Tages aufhalten oder in denen sie Unterkunft erhalten, und die zuständigen Leistungsträger, haben gegenüber dem überörtlichen Träger der Jugendhilfe Anspruch auf Beratung bei der Entwicklung und Anwendung fachlicher Handlungsleitlinien

1. zur Sicherung des Kindeswohls und zum Schutz vor Gewalt sowie

2. zu Verfahren der Beteiligung von Kindern und Jugendlichen an strukturellen Entscheidungen in der Einrichtung sowie zu Beschwerdeverfahren in persönlichen Angelegenheiten.

\section{$\$ 11$ Jugendarbeit}

(1) Jungen Menschen sind die zur Förderung ihrer Entwicklung erforderlichen Angebote der Jugendarbeit zur Verfügung zu stellen. Sie sollen an den Interessen junger Menschen anknüpfen und von ihnen mitbestimmt und mitgestaltet werden, sie zur Selbstbestimmung befähigen und zu gesellschaftlicher Mitverantwortung und zu sozialem Engagement anregen und hinführen.

(2) Jugendarbeit wird angeboten von Verbänden, Gruppen und Initiativen der Jugend, von anderen Trägern der Jugendarbeit und den Trägern der öffentlichen Jugendhilfe. Sie umfasst für Mitglieder bestimmte Angebote, die offene Jugendarbeit und gemeinwesenorientierte Angebote.

(3) Zu den Schwerpunkten der Jugendarbeit gehören:

1. außerschulische Jugendbildung mit allgemeiner, politischer, sozialer, gesundheitlicher, kultureller, naturkundlicher und technischer Bildung,

2. Jugendarbeit in Sport, Spiel und Geselligkeit, 
3. arbeitswelt-, schul- und familienbezogene Jugendarbeit,

4. internationale Jugendarbeit,

5. Kinder- und Jugenderholung,

6. Jugendberatung.

(4) Angebote der Jugendarbeit können auch Personen, die das 27. Lebensjahr vollendet haben, in angemessenem Umfang einbeziehen.

\section{$\$ 12$ Förderung der Jugendverbände}

(1) Die eigenverantwortliche Tätigkeit der Jugendverbände und Jugendgruppen ist unter Wahrung ihres satzungsgemäßen Eigenlebens nach Maßgabe des $\$ 74$ zu fördern.

(2) In Jugendverbänden und Jugendgruppen wird Jugendarbeit von jungen Menschen selbst organisiert, gemeinschaftlich gestaltet und mit verantwortet. Ihre Arbeit ist auf Dauer angelegt und in der Regel auf die eigenen Mitglieder ausgerichtet, sie kann sich aber auch an junge Menschen wenden, die nicht Mitglieder sind. Durch Jugendverbände und ihre Zusammenschlüsse werden Anliegen und Interessen junger Menschen zum Ausdruck gebracht und vertreten.

\section{$\$ 72$ Mitarbeiter, Fortbildung}

(1) Die Träger der öffentlichen Jugendhilfe sollen bei den Jugendämtern und Landesjugendämtern hauptberuflich nur Personen beschäftigen, die sich für die jeweilige Aufgabe nach ihrer Persönlichkeit eignen und eine dieser Aufgabe entsprechende Ausbildung erhalten haben (Fachkräfte) oder auf Grund besonderer Erfahrungen in der sozialen Arbeit in der Lage sind, die Aufgabe zu erfüllen. Soweit die jeweilige Aufgabe dies erfordert, sind mit ihrer Wahrnehmung nur Fachkräfte oder Fachkräfte mit entsprechender Zusatzausbildung zu betrauen. Fachkräfte verschiedener Fachrichtungen sollen zusammenwirken, soweit die jeweilige Aufgabe dies erfordert.

(2) Leitende Funktionen des Jugendamts oder des Landesjugendamts sollen in der Regel nur Fachkräften übertragen werden.

(3) Die Träger der öffentlichen Jugendhilfe haben Fortbildung und Praxisberatung der Mitarbeiter des Jugendamts und des Landesjugendamts sicherzustellen. 


\section{$\$ 72 a$ Tätigkeitsausschluss einschlägig vorbestrafter Personen}

(1) Die Träger der öffentlichen Jugendhilfe dürfen für die Wahrnehmung der Aufgaben in der Kinder- und Jugendhilfe keine Person beschäftigen oder vermitteln, die rechtskräftig wegen einer Straftat nach den $\$ \$ 171,174$ bis $174 \mathrm{c}, 176$ bis $180 \mathrm{a}, 181 \mathrm{a}, 182$ bis $184 \mathrm{~g}$, 225, 232 bis 233a, 234, 235 oder 236 des Strafgesetzbuchs verurteilt worden ist. Zu diesem Zweck sollen sie sich bei der Einstellung oder Vermittlung und in regelmäßigen Abständen von den betroffenen Personen ein Führungszeugnis nach $\$ 30$ Absatz 5 und $\$ 30$ a Absatz 1 des Bundeszentralregistergesetzes vorlegen lassen.

(2) Die Träger der öffentlichen Jugendhilfe sollen durch Vereinbarungen mit den Trägern der freien Jugendhilfe sicherstellen, dass diese keine Person, die wegen einer Straftat nach Absatz 1 Satz 1 rechtskräftig verurteilt worden ist, beschäftigen.

(3) Die Träger der öffentlichen Jugendhilfe sollen sicherstellen, dass unter ihrer Verantwortung keine neben- oder ehrenamtlich tätige Person, die wegen einer Straftat nach Absatz 1 Satz 1 rechtskräftig verurteilt worden ist, in Wahrnehmung von Aufgaben der Kinder- und Jugendhilfe Kinder oder Jugendliche beaufsichtigt, betreut, erzieht oder ausbildet oder einen vergleichbaren Kontakt hat. Hierzu sollen die Träger der öffentlichen Jugendhilfe über die Tätigkeiten entscheiden, die von den in Satz 1 genannten Personen auf Grund von Art, Intensität und Dauer des Kontakts dieser Personen mit Kindern und Jugendlichen nur nach Einsichtnahme in das Führungszeugnis nach Absatz 1 Satz 2 wahrgenommen werden dürfen.

(4) Die Träger der öffentlichen Jugendhilfe sollen durch Vereinbarungen mit den Trägern der freien Jugendhilfe sowie mit Vereinen im Sinne des $\$ 54$ sicherstellen, dass unter deren Verantwortung keine neben- oder ehrenamtlich tätige Person, die wegen einer Straftat nach Absatz 1 Satz 1 rechtskräftig verurteilt worden ist, in Wahrnehmung von Aufgaben der Kinder- und Jugendhilfe Kinder oder Jugendliche beaufsichtigt, betreut, erzieht oder ausbildet oder einen vergleichbaren Kontakt hat. Hierzu sollen die Träger der öffentlichen Jugendhilfe mit den Trägern der freien Jugendhilfe Vereinbarungen über die Tätigkeiten schließen, die von den in Satz 1 genannten Personen auf Grund von Art, Inten- 
sität und Dauer des Kontakts dieser Personen mit Kindern und Jugendlichen nur nach Einsichtnahme in das Führungszeugnis nach Absatz 1 Satz 2 wahrgenommen werden dürfen.

(5) Träger der öffentlichen und freien Jugendhilfe dürfen von den nach den Absätzen 3 und 4 eingesehenen Daten nur den Umstand, dass Einsicht in ein Führungszeugnis genommen wurde, das Datum des Führungszeugnisses und die Information erheben, ob die das Führungszeugnis betreffende Person wegen einer Straftat nach Absatz 1 Satz 1 rechtskräftig verurteilt worden ist. Die Träger der öffentlichen und freien Jugendhilfe dürfen diese erhobenen Daten nur speichern, verändern und nutzen, soweit dies zum Ausschluss der Personen von der Tätigkeit, die Anlass zu der Einsichtnahme in das Führungszeugnis gewesen ist, erforderlich ist. Die Daten sind vor dem Zugriff Unbefugter zu schützen. Sie sind unverzüglich zu löschen, wenn im Anschluss an die Einsichtnahme keine Tätigkeit nach Absatz 3 Satz 2 oder Absatz 4 Satz 2 wahrgenommen wird. Andernfalls sind die Daten spätestens drei Monate nach der Beendigung einer solchen Tätigkeit zu löschen.

\section{A1.2 Bundeszentralregistergesetz}

\section{$\$ 30$ Antrag}

(1) Jeder Person, die das 14. Lebensjahr vollendet hat, wird auf Antrag ein Zeugnis über den sie betreffenden Inhalt des Registers erteilt (Führungszeugnis). Hat der Betroffene einen gesetzlichen Vertreter, so ist auch dieser antragsberechtigt. Ist der Betroffene geschäftsunfähig, so ist nur sein gesetzlicher Vertreter antragsberechtigt.

(2) Wohnt der Antragsteller innerhalb des Geltungsbereichs dieses Gesetzes, so ist der Antrag bei der Meldebehörde zu stellen; sofern der Antragsteller nicht persönlich erscheint, ist eine schriftliche Antragstellung mit amtlich oder öffentlich beglaubigter Unterschrift des Antragstellers zulässig. Der Antragsteller hat seine Identität und, wenn er als gesetzlicher Vertreter handelt, seine Vertretungsmacht nachzuweisen. Der Betroffene und sein gesetzlicher Vertreter können sich bei der Antragstellung nicht 
durch einen Bevollmächtigten vertreten lassen. Die Meldebehörde nimmt die Gebühr für das Führungszeugnis entgegen, behält davon zwei Fünftel ein und führt den Restbetrag an die Bundeskasse ab.

(3) Wohnt der Antragsteller außerhalb des Geltungsbereichs dieses Gesetzes, so kann er den Antrag unmittelbar bei der Registerbehörde stellen. Absatz 2 Satz 2 und 3 gilt entsprechend.

(4) Die Übersendung des Führungszeugnisses an eine andere Person als den Antragsteller ist nicht zulässig.

(5) Wird das Führungszeugnis zur Vorlage bei einer Behörde beantragt, so ist es der Behörde unmittelbar zu übersenden. Die Behörde hat dem Antragsteller auf Verlangen Einsicht in das Führungszeugnis zu gewähren. Der Antragsteller kann verlangen, daß das Führungszeugnis, wenn es Eintragungen enthält, zunächst an ein von ihm benanntes Amtsgericht zur Einsichtnahme durch ihn übersandt wird. Die Meldebehörde hat den Antragsteller in den Fällen, in denen der Antrag bei ihr gestellt wird, auf diese Möglichkeit hinzuweisen. Das Amtsgericht darf die Einsicht nur dem Antragsteller persönlich gewähren. Nach Einsichtnahme ist das Führungszeugnis an die Behörde weiterzuleiten oder, falls der Antragsteller dem widerspricht, vom Amtsgericht zu vernichten.

(6) Wohnt der Antragsteller außerhalb des Geltungsbereichs dieses Gesetzes, so kann er verlangen, daß das Führungszeugnis, wenn es Eintragungen enthält, zunächst an eine von ihm benannte amtliche Vertretung der Bundesrepublik Deutschland zur Einsichtnahme durch ihn übersandt wird. Absatz 5 Satz 5 und 6 gilt für die amtliche Vertretung der Bundesrepublik Deutschland entsprechend.

\section{$\$ 30$ a Antrag auf ein erweitertes Führungszeugnis}

(1) Einer Person wird auf Antrag ein erweitertes Führungszeugnis erteilt,

1. wenn die Erteilung in gesetzlichen Bestimmungen unter Bezugnahme auf diese Vorschrift vorgesehen ist oder

2. wenn dieses Führungszeugnis benötigt wird für

a) die Prüfung der persönlichen Eignung nach $\$ 72$ a des Achten Buches Sozialgesetzbuch - Kinder- und Jugendhilfe -, 
b) eine sonstige berufliche oder ehrenamtliche Beaufsichtigung, Betreuung, Erziehung oder Ausbildung Minderjähriger oder

c) eine Tätigkeit, die in einer Buchstabe bvergleichbaren Weise geeignet ist, Kontakt zu Minderjährigen aufzunehmen.

(2) Wer einen Antrag auf Erteilung eines erweiterten Führungszeugnisses stellt, hat eine schriftliche Aufforderung vorzulegen, in der die Person, die das erweiterte Führungszeugnis vom Antragsteller verlangt, bestätigt, dass die Voraussetzungen nach Absatz 1 vorliegen. Im Übrigen gilt $\$ 30$ entsprechend.

\section{A1.3 Bürgerliches Gesetzbuch}

\section{$\$ 163$ Inhalt und Grenzen der Personensorge}

(1) Die Personensorge umfasst insbesondere die Pflicht und das Recht, das Kind zu pflegen, zu erziehen, zu beaufsichtigen und seinen Aufenthalt zu bestimmen.

(2) Kinder haben ein Recht auf gewaltfreie Erziehung. Körperliche Bestrafungen, seelische Verletzungen und andere entwürdigende Maßnahmen sind unzulässig.

(3) Das Familiengericht hat die Eltern auf Antrag bei der Ausübung der Personensorge in geeigneten Fällen zu unterstützen.

\section{$\$ 1666$ Gerichtliche Maßnahmen bei Gefährdung des Kindeswohls}

(1) Wird das körperliche, geistige oder seelische Wohl des Kindes oder sein Vermögen gefährdet und sind die Eltern nicht gewillt oder nicht in der Lage, die Gefahr abzuwenden, so hat das Familiengericht die Maßnahmen zu treffen, die zur Abwendung der Gefahr erforderlich sind.

(2) In der Regel ist anzunehmen, dass das Vermögen des Kindes gefährdet ist, wenn der Inhaber der Vermögenssorge seine Unterhaltspflicht gegenüber dem Kind oder seine mit der Vermögenssorge verbundenen Pflichten verletzt oder Anordnungen des Gerichts, die sich auf die Vermögenssorge beziehen, nicht befolgt.

(3) Zu den gerichtlichen Maßnahmen nach Absatz 1 gehören insbesondere 
1. öffentliche Hilfen wie zum Beispiel Leistungen der Kinderund Jugendhilfe und der Gesundheitsfürsorge in Anspruch zu nehmen,

2. Gebote, für die Einhaltung der Schulpflicht zu sorgen,

3. Verbote, vorübergehend oder auf unbestimmte Zeit die Familienwohnung oder eine andere Wohnung zu nutzen, sich in einem bestimmten Umkreis der Wohnung aufzuhalten oder zu bestimmende andere Orte aufzusuchen, an denen sich das Kind regelmäßig aufhält,

4. Verbote, Verbindung zum Kind aufzunehmen oder ein $\mathrm{Zu}$ sammentreffen mit dem Kind herbeizuführen,

5. die Ersetzung von Erklärungen des Inhabers der elterlichen Sorge,

6. die teilweise oder vollständigeEntziehung der elterlichen Sorge.

(4) In Angelegenheiten der Personensorge kann das Gericht auch Maßnahmen mit Wirkung gegen einen Dritten treffen.

\section{A2 Auszug aus dem Strafgesetzbuch - Straftaten, die zu einem Tätigkeitsausschluss nach §72a Abs. 1 Satz 1 SGB VIII führen}

Sofern im Führungszeugnis eine der genannten Straftatbestände eingetragen ist, hat dies einen Tätigkeitsausschluss zur Folge.

Straftaten gegen den Personenstand, die Ehe und die Familie $\$ 171$ Verletzung der Fürsorge- oder Erziehungspflicht

Straftaten gegen die sexuelle Selbstbestimmung

$\$ 174$ Sexueller Missbrauch von Schutzbefoblenen

$\$ 174$ a Sexueller Missbrauch von Gefangenen, behördlich Verwahrten oder Kranken und Hilfsbedürftigen in Einrichtungen

$\$ 174 b$ Sexueller Missbrauch unter Ausnutzung einer Amtsstellung

$\$ 174$ c Sexueller Missbrauch unter Ausnutzung eines Beratungs-, Behandlungs- oder Betreuungsverhältnisses

$\$ 176$ Sexueller Missbrauch von Kindern

$\$ 176$ a Schwerer sexueller Missbrauch von Kindern 
$\$ 176 b$ Sexueller Missbrauch von Kindern mit Todesfolge

$\$ 177$ Sexuelle Nötigung; Vergewaltigung

$\$ 178$ Sexuelle Nötigung und Vergewaltigung mit Todesfolge

$\$ 179$ Sexueller Missbrauch widerstandsunfähiger Personen

$\$ 182$ Sexueller Missbrauch von Jugendlichen

$\$ 183$ Exhibitionistische Handlungen

$\$ 183$ a Erregung öffentlichen Ärgernisses

$\$ 184$ Verbreitung pornographischer Schriften

$\$ 184$ a Verbreitung gewalt-oder tierpornographischer Schriften

$\$ 184 b$ Verbreitung, Erwerb und Besitz kinderpornographischer Schriften

$\$ 184$ c Verbreitung, Erwerb und Besitzjugendpornographischer Schriften $\$ 184 d$ Verbreitung pornographischer Darbietungen durch Rundfunk, Medien-oder Teledienste

$\$ 184$ e Ausübung der verbotenen Prostitution

$\$ 184 f$ Jugendgefährdende Prostitution

Straftaten gegen die körperliche Unversehrtheit

$\$ 225$ Misshandlung von Schutzbefoblenen

Straftaten gegen die persönliche Freiheit (Achtzehnter Abschnitt)

$\$ 232$ Menschenhandel zum Zweck der sexuellen Ausbeutung

$\$ 233$ Menschenhandel zum Zweck der Ausbeutung der Arbeitskraft

$\$ 233$ a Förderung des Menschenhandels

$\$ 234$ Menschenraub

$\$ 235$ Entziehung Minderjähriger

$\$ 236$ Kinderhandel 


\section{A3 Auszug aus der Vereinbarung zur Sicherstellung des Schutzauftrags ${ }^{8}$}

\begin{tabular}{|c|c|}
\hline $\begin{array}{l}\text { 6. »insoweit } \\
\text { erfahrene } \\
\text { Fachkraft: }\end{array}$ & $\begin{array}{l}\text { Die nach } \$ 8 \text { a SGB VIII hinzuzuziehende »insoweit er- } \\
\text { fahrene« Fachkraft soll über entsprechende Erfahrun- } \\
\text { gen in der Einschätzung von Gefährdungssituationen für } \\
\text { Kindeswohl verfügen. Bei Verdacht auf sexuellen Miss- } \\
\text { brauch sind andere Kompetenzen notwendig als bei Ver- } \\
\text { dacht körperlicher bzw. gesundheitlicher Vernachlässi- } \\
\text { gung. Die insoweit erfahrene Fachkraft sollte in gewis- } \\
\text { ser Regelmäßigkeit mit Kinderschutzfragen tatsächlich } \\
\text { befasst sein. } \\
\text { Eine Fachkraft (im Sinne des } § 72 \text { SGB VIII) sollte über } \\
\text { folgende Kompetenzen verfügen, um als »insoweit er- } \\
\text { fahrene« Fachkraft im Sinne des } § 8 \text { a SGB VIII tätig zu } \\
\text { sein: } \\
\text { - Kenntnisse über Familiensysteme und die Dynamik } \\
\text { konflikthafter Beziehungen } \\
\text { - Grundbedürfnisse von Kindern und Jugendlichen in } \\
\text { den jeweiligen Entwicklungsphasen } \\
\text { - Symptome und Entwicklungsbeeinträchtigungen von } \\
\text { Kindern und Jugendlichen in gefährdenden Beziehungen } \\
\text { - Bindungsverhalten und -bedürfnisse von Kindern } \\
\text { - Risikobehaftete Lebenslagen von Familien } \\
\text { - Kenntnis des rechtlichen Rahmens von Kindeswohl- } \\
\text { gefährdung und Datenschutz } \\
\text { - Kenntnis des Hilfesystems und der Kooperationswege } \\
\text { - Methodische Kenntnisse zur kollegialen Beratung }\end{array}$ \\
\hline
\end{tabular}

\section{A4 Signale von Kindeswohlgefährdung}

So vielfältig die Signale sein können, so vielfältig sind sie auch in der Literatur zu finden. Mehrfach findet man aber folgende Signale bei den betroffenen Kindern (vgl. unter anderem Moggi, 2005, S. 96f.):

Körperlich:

> falsche oder unzureichende Ernährung(Über-oder Untergewicht)

$>$ unangenehmer Geruch, starker Mundgeruch durch mangelnde Zahnhygiene 
$>$ chronische Müdigkeit, oft fehlende witterungsgerechte Kleidung

$>$ unversorgte Wunden, Hämatome, Narben, Krankheitsanfälligkeit

$>$ starke und auffällige körperliche Entwicklungsverzögerungen

Kognitiv:

$>$ motorische, kognitive emotionale oder soziale Entwicklungsrückstände

$>$ Wahrnehmungs- und Gedächtnisstörungen

$>$ ausgeprägte Konzentrationsschwäche

$>$ Verzögerung in der Sprach- sowie in der altersgemäßen Intelligenzentwicklung

Psychisch:

> Apathie, Traurigkeit, Aggressivität, Schreckhaftigkeit, Unruhe, Schüchternheit, Ängstlichkeit, Verschlossenheit, Verlustängste

$>$ Schuldgefühle für das Verhalten der Eltern oder anderer Bezugspersonen

$>$ fehlendes Bindungsverhalten gegenüber ihren Betreuungspersonen (Loyalitätskonflikte gegenüber den Eltern)

$>$ Schlafstörungen, Essstörungen, Einnässen, Einkoten, Stottern

$>$ selbstverletzendes Verhalten

Soziales Verhalten:

$>$ extrem überangepasstes Verhalten

$>\quad$ Weglaufen, straffälliges Verhalten, Lügen

$>$ Gewaltanwendungen im Konfliktfall

$>$ unangemessenes sexualisiertes Verhalten

$>\quad$ das Kind oder der Jugendliche will nicht nach Hause gehen

$>$ Schulschwierigkeiten, Schulschwänzen

$>$ Bericht über Gewalttätigkeiten in der Familie

> plötzlich keine Lust mehr, an der Gruppenstunde teilzunehmen, ohne erkennbaren Grund

$>$ Meidung bestimmter Orte oder Personen, oft in Verbindung mit abschätzigen Kommentaren

Des Weiteren gibt es auch Merkmale im Umfeld des Kindes, die auf eine Gefährdung hinweisen können: 
Verhalten von wichtigen Bezugspersonen:

$>\quad$ aggressives, aufbrausendes Verhalten gegenüber dem Kind

$>$ massive Beschimpfung oder Erniedrigung

$>$ ständiges distanziertes Verhalten dem Kind gegenüber

$>$ ermöglicht Zugang zu nicht altersgemäßen Medien (Gewalt verherrlichende Videos, Computerspiele oder pornografische Schriften)

$>$ Förderung oder Behandlung zum Beispiel des behinderten Kindes wird verweigert

Wohnsituation:

$>\quad$ kein eigener Schlafplatz für das Kind

$>$ stark verschmutze Wohnung

$>$ keine Privatsphäre

$>$ kein altersgerechtes Spielzeug

\section{A5 Dokumentationsblatt bezüglich der Einsichtnahme in das Führungszeugnis bei neben- oder ehrenamtlich tätigen Personen}

Gesetzliche Grundlage der Dokumentation: §72a Abs. 5 SGB VIII

Dokumentiert werden darf (vgl. Wiesner, 2015, \$72a Rn. 48)

1. bei vorliegendem Tätigkeitsausschluss:

$>$ Name der Person

$>$ die Tatsache, dass ein Tätigkeitsausschluss vorliegt, sofern die Tätigkeit schon aufgenommen wurde

$>$ das Datum der Einsichtnahme

$>$ die Löschung dieser Dokumentation erfolgt:

$>$ sofort, wenn die Tätigkeit, aufgrund dessen das Führungszeugnis beantragt wurde, nicht aufgenommen wird

> spätestens drei Monate nach Beendigung der Tätigkeit, aufgrund dessen das Führungszeugnis beantragt wurde

2. bei keinem Eintrag in das erweiterte Führungszeugnis:

$>$ Name der Person

$>$ Datum der Einsichtnahme

$>$ Datum der nächsten Einsichtnahme 


\begin{tabular}{|c|c|c|c|c|}
\hline $\begin{array}{c}\text { Vor- und } \\
\text { Nachname der } \\
\text { neben- und } \\
\text { ehrenamtlich } \\
\text { tätigen Person }\end{array}$ & $\begin{array}{c}\text { Datum der } \\
\text { Einsichtnahme } \\
\text { des erweiterten } \\
\text { Führungs- } \\
\text { zeugnisses }\end{array}$ & $\begin{array}{c}\text { Es liegt ein } \\
\text { Tätigkeitsaus- } \\
\text { schluss gemäß } \\
\text { \$72a SGB VIII } \\
\text { vor }\end{array}$ & $\begin{array}{c}\text { Unterschrift, } \\
\text { des/der } \\
\text { Einsicht- } \\
\text { nehmenden }\end{array}$ & $\begin{array}{c}\text { Datum der } \\
\text { nächsten } \\
\text { Einsichtnahme }\end{array}$ \\
\hline & & $\square \mathrm{Ja}$ & & \\
\hline & & $\square \mathrm{Ja}$ & & \\
\hline & & $\square \mathrm{Ja}$ & & \\
\hline & $\square \mathrm{Ja}$ & & \\
\hline & $\square \mathrm{Ja}$ & & \\
\hline & $\square \mathrm{Ja}$ & & \\
\hline & $\square \mathrm{Ja}$ & & \\
\hline & & $\square \mathrm{Ja}$ & & \\
\hline & & $\square \mathrm{Ja}$ & & \\
\hline & & $\square \mathrm{Ja}$ & & \\
\hline & & & & \\
\hline
\end{tabular}

Die Daten dürfen nicht übermittelt werden und sind vom Zugriff Dritter zu schützen!

\section{A6 Dokumentation - Vermutungstagebuch - Fragestellungen}

Eine Dokumentation ist ab dem Zeitpunkt der Beobachtung bzw. des Berichtes sinnvoll und nützlich. Sie dient der eigenen Absicherung und Gedächtnisstütze sowie der Vergewisserung der Beobachtung oder des Gehörten. Nicht immer sind die Beobachtungen und Erzählungen eindeutig einer Straftat zuzuordnen. Das eigene Bauchgefühl ist aber ernst zu nehmen und eine Dokumentation bzw. ein Vermutungstagebuch (vgl. PräTect) kann hilfreich sein.

Eine Dokumentation beinhaltet immer:

$>\quad$ das Datum und den Anlass der Vermutung für das Vorliegen sexualisierter Gewalt oder Kindeswohlgefährdung

$>$ Beobachtungen und Berichte (diese, wenn möglich, in wörtlicher Rede)

$>$ Namen und Kontakt der berichtenden Person

$>$ Beteiligte am Gespräch bzw. der Beobachtung

$>$ informierte Stellen

> was besprochen wurde, welche Absprachen bezüglich des weiteren Vorgehens getroffen wurden 
$>$ eventuelle Empfehlungen von externer Stelle

$>$ Begründung für die Entscheidung(en)

Neben der Sachdokumentation ist eine eigene Reflexionsdokumentation hilfreich, die eigenen Gefühle und Gedanken zu ordnen:

$>$ Welche Gefühle lösen die Beobachtungen oder das Gehörte bei mir aus?

$>$ Habe ich Erklärungen für das Geschehen?

$>$ Was sollten meine nächsten Schritte sein?

Mehr Anregungen gibt es in den Handlungsleitfäden.

\section{A7 Empfehlenswerte Handlungsleitfäden}

Bayerischer Landesjugendring \& Prätect-Arbeitshilfe (April 2013). Handeln bei Verdacht auf sexuelle Gewalt in der Jugendarbeit. Krisenmanagement, Öffentlichkeitsarbeit, Rechtsfragen. München. Sehr gute Arbeitshilfe mit allen Hinweisen und verschiedenen Fallbesprechungen. U.a. auch der Umgang mit Presse.

Bund Deutscher katholischer Jugend (BDKJ) \& Bischöfliches Jugendamt (BJA) der Diözese Rottenburg-Stuttgart (2011). Was tun ...? ... Bei (Verdacht auf) Kindesmisshandlung, sexueller Gewalt oder Vernachlässigung? Handlungsempfehlungen für ehrenamtliche und hauptberufliche MitarbeiterInnen im BDKJ/BJA Rottenburg-Stuttgart (2. Aufl.). Rottenburg-Stuttgart.

Ein guter Handlungsleitfaden für ehrenamtlich und hauptberuflich Beschäftigte. Antworten und Vorschläge für Reaktionen auf bestimmte Grenzverletzungen und konkrete Verdachts- und Notfälle. Mit einem ergänzenden Hinweis für hauptberufliche Mitarbeitende und Adressen und konkrete Ansprechpartner im Bereich Baden-Württemberg.

Bund Deutscher katholischer Jugend (BDKJ) Nordrhein-Westfalen (2012). Kinder schützen. Eine Information für Gruppenleiter/innen verbandlicher Jugendgruppen (4., überarb. Aufl.). Münster. 
Diese Information ist eine übersichtliche Zusammenstellung für ehrenamtliche Gruppenleiter zum Thema Kindeswohlgefährdung und dem Umgang damit.

Evangelische Kirche von Kurhessen-Waldeck (Mai 2012). Handlungsleitfaden zum Kindesschutz für hauptberuflich Beschäftigte und Verantwortungsträger in der Kinder- und Jugendarbeit der Evangelischen Kirche von Kurhessen-Waldeck. Kassel.

Ein guter und umfassender Handlungsleitfaden des Referats Kinderund Jugendarbeit. Der umfangreiche Anhang beinhaltet unter anderem Beobachtungs- und Bewertungsinstrumente zur Risiko- und Gefahreneinschätzung, einen Verhaltenskodex und eine Selbstverpflichtungserklärung sowie Gruppenstundenentwürfe für die Arbeit mit Kindern und Jugendlichen.

Landesjugendring Schleswig-Holstein (2010).»/rgendetwasstimmt da nicht «. Kiel.

Ein sehr zu empfehlender, umfassender Leitfaden für Ehrenamtliche zum Umgang mit dem Schutzauftrag bei Kindeswohlgefährdung.

Verband christlicher Pfadfinderrinnen und Pfadfinder (VCP) (2010). AKTIV! gegen sexualisierte Gewalt. Eine Handreichung für Verantwortungsträgerinnen und -träger im VCP (2., leicht überarb. Aufl.). Kassel.

Zielgruppe dieser Handreichung sind alle Verantwortungsträger im VCP. Erklärtes Ziel ist die Schärfung des Blickes und die Sensibilisierung der Wahrnehmung auf allen Ebenen des Verbandes. Die Entwicklung der Handreichung erfolgte von einer dafür gegründeten Arbeitsgruppe, deren Aufgabe es ist, das Thema in allen Bereichen zu verankern.

\section{A8 Bausteine für einen Handlungsleitfaden}

Ein Handlungsleitfaden sollte so aufgebaut sein, dass er schlüssig und klar beschreibt, was für die Mitarbeitenden wichtig ist. Das bedeutet auch, dass es für die unterschiedlichen Fälle auch entsprechende 
Handlungsoptionen geben muss. Ein erster Schritt ist daher die Unterscheidung der Ausgangslage:

1. Handlungsoptionen bei (sexuellem) Machtmissbrauch, der vonseiten einer_s hauptamtlich Mitarbeitenden gegenüber Teilnehmenden oder ehrenamtlich Mitarbeitenden ausgeht

2. Handlungsoptionen bei (sexuellem) Machtmissbrauch, der vonseiten einer_s ehrenamtlich Mitarbeitenden gegenüber Teilnehmenden ausgeht

3. Handlungsoptionen bei einem Verdacht auf Kindeswohlgefährdung im engen Umfeld des Kindes, ohne Beteiligung einer_s Mitarbeitenden des Verbandes

4. Handlungsoptionen bei Grenzüberschreitungen und (sexuellem) Machtmissbrauch innerhalb der Teilnehmerschaft

Anhand eines solchen Leitfadens kann auch die Seminargestaltung zum Modul Kindeswohlgefährdung, Grenzüberschreitungen und (sexueller) Machtmissbrauch aufgebaut werden. Ein Leitfaden sollte Informationen liefern und die Frage nach den Handlungsmöglichkeiten klar beantworten. Wichtige Inhalte sind demnach:

$>\quad$ einleitende Worte zum Schutzauftrag und dem Selbstverständnis in der verbandlichen Jugendarbeit

$>\quad »$ Abholen « der Mitarbeitenden in der Situation: »Ich hab' da so ein Bauchgefühl ...«; Eingehen auf dieses diffuse, beunruhigende Gefühl über verschiedene Zugänge: selbst beobachtet, Kind oder Eltern haben etwas erzählt oder auch eigene Vermutungen; Wichtigkeit der eigenen Gefühle unterstreichen

$>$ Benennung möglicher Anhaltspunkte mit dem Hinweis, dass diese aber nicht automatisch ein Anzeichen sind (siehe entsprechendes Kapitel)

$>$ Erscheinungsbild des Kindes

$>$ Verhalten des Kindes

$>$ Verhalten der Eltern

$>$ eventuelle Wohnsituation

$>$ konkrete Schritte bei einer Vermutung, am sinnvollsten mittels eines Verlaufsdiagrammes

$>\quad$ Verhalten bei einer Vermutung

$>$ Ruhe bewahren 
$>$ Vermutungstagebuch (vgl. Material PräTect)

$>$ Austausch mit dem_der Teamkolleg_in und hauptberuflicher Person des Vertrauens

$>$ Einbeziehung des Vorstandes/ der Leitung

$>$ Hinzuziehen einer Fachkraft

$>$ konkrete Schritte bei Anvertrauen

$>$ Ruhe bewahren

$>$ keine Versprechungen, die man nicht halten kann (z. B. mit niemanden darüber reden)

$>$ dem Kind Glauben schenken

$>$ seine Not ernst nehmen

$>$ nicht über seinem Kopf hinweg Entscheidungen treffen

$>$ keine Konfrontation gegenüber dem_der Täter_in

$>$ vertrauliche Behandlung des Themas

$>$ >Tagebuch «

$>$ Kontaktaufnahme mit Vertrauensperson oder Fachkraft

$>$ konkrete Schritte bei Verdacht in den eigenen Reihen

$>$ Beobachtungen notieren

$>$ Kontaktaufnahme zu einer Vertrauensperson

$>$ Information der Fachkraft im Verband

> Umgang mit sexuellen Übergriffen unter Kindern und Jugendlichen

$>$ klare Stellung beziehen

$>$ parteiliche Haltung für das betroffene Kind

$>$ übergriffigem Kind/Jugendlichen die Ablehnung des falschen Verhaltens erklären, ohne jedoch die Person an sich abzulehnen

$>$ Kontaktaufnahme zur Fachkraft (Eltern müssen informiert werden, das sollte jedoch mit Unterstützung der hauptberuflich Beschäftigten erfolgen)

$>$ bei Jugendlichen muss auch die Polizei hinzugezogen werden $(\$ \$ 176,177$ StGB in Verbindung mit $\$ 13$ StGB)

$>\quad$ Umgang mit Täter_innen (die auch Opfer sein können)

$>$ Ansprechpartner im Verband

> konkrete Personen, an die sich Kinder, Jugendliche, Ehrenamtliche, Eltern und Fachkräfte wenden können

$>$ wichtige Rufnummern/Telefonhotline für Freizeitteams

$>$ Kinder- und Jugendtelefon 
$>$ Telefonhotline für Freizeitteams (Notfallnummer)

$>$ Hinweise zu Beratungsstellen

$>$ allgemeine Beratungsstellen wie Kinderschutzbund, N.I.N.A, Zartbitter e.V.

$>$ konkrete Beratungsstellen aus dem städtischen Umfeld für kurze Wege

$>$ eventuell Beispiel für ein »Vermutungstagebuch « und Dokumentation

\section{A9 Impulse zur Erstellung eines Verhaltenskodex}

In der Jugendarbeit übernehmen Leitungskräfte in vielfacher Weise Verantwortung für das Wohl der ihnen anvertrauten Kinder und Jugendlichen. Ein Ziel ist dabei der weitestgehende Schutz der Kinder, Jugendlichen und Jugendleiter_innen vor sexuellen Übergriffen, sexualisierter Atmosphäre sowie geschlechtsspezifischen Diskriminierungen.

Ein Verhaltenskodex steht in Bezug zu gesetzlichen Bestimmungen (Bsp. §8a, §72a SGB VIII) und beinhaltet eine Selbstverpflichtung und Ziele zur Prävention sexueller Gewalt in der Kinder- und Jugendarbeit. Er dient der Erleichterung, dem sicheren Umgang und dem Erwerb einer klaren Haltung zur Prävention vor sexualisierter Gewalt. Er ist eine Willenserklärung für zukünftiges Verhalten, die auch eine regelmäßige Überprüfung und Reflexion erfordert. Ebenso schützt ein Verhaltenskodex vor Missverständnissen und falschem Verdacht, denn wenn Mitarbeitende ihr Handeln nach diesem ausrichten, können sie sich vor verfänglichen Situationen schützen. Die gemeinsame Erarbeitung und Auseinandersetzung eines Verhaltenskodex hilft, das Thema ins Bewusstsein der Mitarbeitenden zu bringen und die aktive Auseinandersetzung zu fördern. Das erhöht die Aufmerksamkeit gegenüber Grenzüberschreitungen. Nach außen symbolisiert ein Verhaltenskodex mit angeschlossener Selbstverpflichtung Eltern und Interessierten an der Arbeit des Jugendverbandes, dass sich Mitarbeitende einem präventiven Verhalten und dem Wohle der Kinder und Jugendlichen verpflichten. Die Entwicklung eines Verhaltenskodex gehört zum Standard von Präventionskonzepten (vgl. UBSKM). 
Im Folgenden werden Hinweise und Fragestellungen gegeben, die bei der Erarbeitung und Diskussion eines eigenen Verhaltenskodex hilfreich sein können. Sie sind Bestandteil der meisten Kodizes.

\section{a. Schutzbefoblene bzw. anvertraute Personen}

Vor der Erstellung eines Verhaltenskodex ist der Geltungsbereich zu bestimmen. Für welche Situationen gilt dieser Verhaltenskodex?

$>$ Freizeitarbeit

$>$ Kinder- und Jugendarbeit

$>$ Seminararbeit

b. Welche Grundhaltung haben wir? Was macht unsere Arbeit aus (wird oft als Einleitung oder Grundlegung an den Anfang gestellt)?

$>$ bei kirchlichen Verbänden:

$>$ Wurzeln im christlichen Glauben

$>$ christliches Menschenbild

$>$ Achtung der Würde des Gegenübers

$>$ Achtung der Autonomie des Gegenübers

$>$ Stärkung und Schutz des Gegenübers

$>$ Verhinderung von sexualisierter und anderer Gewalt

$>$ keine Tabuisierung

$>$ klare Verhaltensregeln

$>$ Vorbildfunktion

\section{c. Verhalten gegenüber den anvertrauten Menschen}

Grundfrage: Wie verhalten wir uns in bestimmten Situationen, die typisch für das Handlungsfeld sind? Worauf einigen wir uns?

> Intimsphäre und persönliche Schamgrenzen von Teilnehmenden und Mitarbeitenden werden respektiert

$>$ Eingreifen bei Anzeichen von sexistischem, diskriminierendem und gewalttätigem Verhalten (verbal und nonverbal)

> aktive Stellungnahme bei Grenzüberschreitungen durch Dritte

$>$ Handlungskonzept bei Verdacht auf Kindeswohlgefährdung

$>$ Betreten von Schlafräumen durch Mitarbeitende

$>$ Wie sehen die Duschsituationen aus, z. B. auf Zeltlagern?

$>$ Schwimmbadbesuche, wie verhalten sich die Mitarbeitenden? 
$>\quad$ Wo und wie schlafen Mitarbeitende?

$>$ Trennung von beruflichen und privaten Kontakten

\section{d. Umgang im Team (Kollegialität)}

Grundfrage: Wie verhalten wir uns im Team?

$>$ Wie ist der Umgang untereinander (vertrauensvoll, ehrlich, klar)?

$>$ Was beinhalten die Gespräche?

$>$ Kritik ausschließlich im persönlichen und direkten Kontakt äußern

$>$ konstruktive Kritik

$>$ bei Konflikten Lösungen suchen, und zwar im gemeinsamen Gespräch oder Arbeitskreis

$>$ professionelle/kompetente Unterstützung bei Vermutung

$>\quad$ Nähe-Distanz-Verhältnis von Mitarbeitenden untereinander

$>\quad$ z.B. als Paar auf der Freizeit, im Arbeitsfeld ...

$>$ sonstige Hinweise

Wichtig ist auch, dass deutlich wird, welche Konsequenzen eine Zuwiderhandlung gegen die formulierten Punkte nach sich zieht (vgl. EKD »Auf Grenzen achten - Sicheren Ort geben «, S. 33ff.).

$>$ Was ist unser Beitrag zur Prävention sexualisierter Gewalt im Handlungsfeld?

e. Stichworte, die bei der Erstellung einer anschließenden Selbstverpflichtungserklärung hilfreich sein können

Die Selbstverpflichtungserklärung bezieht sich aufden Verhaltenskodex im Arbeitsfeld. Dazu können folgende Hinweise bei der Formulierung helfen:

Grundfrage: Haltung/Selbstverständnis/Professionalität

$>\quad$ eigene Reflexion und Selbstverständnis

$>$ keine Befriedigungvon persönlichen oder emotionalen Bedürfnissen

$>$ keine Tabuisierung der Bereiche sexualisierter Gewalt und Grenzverletzungen

$>$ kein Machtmissbrauch

$>$ Reflexion von individuellem Nähe-Distanz-Verständnis

$>$ kein abwertendes Verhalten/Sprache 
> eigene Wahrnehmung schärfen

$>$ Schweigepflicht (nicht Vertuschung!)

f. Abgrenzung bzw. Rollenklarheit

$>$ Hauptamt

$>$ Ehrenamt

$>$ Fachkraft

\section{g. Fortbildung}

Grundfrage: Wie wollen wir die Präsenz des Themas erhalten?

$>\quad$ regelmäßige Fortbildungen

$>$ Supervision, Coaching

$>$ Beratung durch Fachkräfte einholen

\section{b. Verortung und Verantwortung}

$>$ Strukturelle Einbindung klar definieren.

$>$ Wer sind die Verantwortlichen im Arbeitsbereich?

$>$ Ansprechpartner

> Handlungskonzept (Notfallplan)

\section{i. Unterschrift und weitere Info}

Um der Selbstverpflichtung einen wichtigen Stellenwert zu geben, der ähnlich der Einsichtnahme in ein erweitertes Führungszeugnis entspricht, kann folgender Abschnitt vor dem Unterschriftsfeld eingefügt werden:

$\gg$ Mit meiner Unterschrift bringe ich zum Ausdruck, dass ich diesen Verhaltenskodex unterstütze und mein Möglichstes dazu beitragen möchte, gegen Grenzverletzungen jeglicher Art aktiv zu werden.

Zudem bestätige ich, dass gegen mich kein Verfahren wegen einer Straftat nach den $\$ \$ 171,174$ bis $174 \mathrm{c}, 176$ bis $181 \mathrm{a}, 182$ bis $184 \mathrm{e}$, 225, 232 bis 236 des Strafgesetzbuches anhängig ist. Ich verpflichte mich, die Verantwortlichen ... (Einrichtung, Gemeinde o.̈.. einfügen) ... sofort zu informieren, wenn ein Verfahren wegen Verstoßes nach den oben genannten Paragrafen gegen mich eröffnet werden sollte. «

Datum, Unterschrift, Name in Druckbuchstaben, Arbeitsbereich 


\section{A10 Empfehlenswerte Hand- und Methodenbücher}

Arbeitsgemeinschaft der Evangelischen Jugendarbeit in Deutschland e.V. (Hrsg.). (2011). Sex. Sex! Sex? Umgang mit Sexualität und sexueller Gewalt bei internationalen Begegnungen, Kinder- und Jugendreisen. Hannover.

Eine gute Schulungsmappe der Arbeitsgemeinschaft der Evangelischen Jugendarbeit in Deutschland e.V. Besonders erwähnenswert ist die Herausstellung von Sexualität als einem positiven Lernfeld für Kinder und Jugendliche. Die zweite Auflage wurde überarbeitet und um das Thema Täter_innen und -strategien erweitert. Auf einer beiliegenden CD befinden sich alle Materialien und Arbeitsblätter, die sich so gut ausdrucken lassen.

Bayerischer Jugendring: PräTect. Material zum Herunterladen unter: www.praetect.de

Prävention vor sexueller Gewalt in der Kinder- und Jugendarbeit. Es gibt mehrere Bausteine. Den Baustein 3 halte ich für die Auseinandersetzung mit Grundlagen und Methoden präventiver Arbeit als sehr gut.

Bund Deutscher Katholischer Jugend Diözesanverband Freiburg (2012). Schutz vor sexueller Gewalt auf Freizeiten. Informationen Anregungen - Arbeitsmaterialien. Freiburg.

Ein Ordner mit vielen Materialien für die Schulungsarbeit und die Thematisierung auf Ferienfreizeiten. Besonders toll sind die Wimmelbilder, die sich sowohl in der Arbeit mit Kindern als auch in der Schulungsarbeit einsetzen lassen.

Evangelische Jugendarbeit in Bayern (2004). „Bei uns nicht! Gemeinsam gegen sexuellen Missbrauch im Jugendverband. Nürnberg.

Sehr umfassend und informativ, allerdings nicht aktuell, da es nicht den $\$ 8$ a SGB VIII aufgreift. Dennoch sind die Grundlagen, Methoden, Übungen, Spiele und Medien sehr umfassend und geben wichtige Hinweise auf den Umgang mit dem Thema. 
Evangelischer Kirchenkreis Köln-Nord (2012). Thema Sexualität. Stärken - begleiten - informieren. Sexualpädagogik in der evangelischen Kinder- und Jugendarbeit. Köln.

Das sexualpädagogische Konzept des Evangelischen Kirchenkreises Köln-Nord umfasst einige Ideen für die konkrete Arbeit mit unterschiedlichen Zielgruppen der Kinder- und Jugendarbeit.

Katholische junge Gemeinde, Bundesverband (2010). Erste allgemeine Verunsicherung. Sexualpädagogik in der KJG. Düsseldorf.

Eine Sammlung von tollen Methoden inklusive der Auseinandersetzung mit dem Thema in der katholischen Jugendarbeit. Empfehlenswert auch die rechtliche Auseinandersetzung.

Timmermanns, Stefan \& Tuider, Elisabeth (2008). Sexualpädagogikder Vielfalt. Praxismethoden zu Identitäten, Beziehungen, Körper und Prävention für Schule und Jugendarbeit. Weinheim: Beltz Juventa.

Ein vielfältiges Methodenbuch für die Praxis.

\section{A11 Methoden und Arbeitsblätter für die Schulung von ehrenamtlich Mitarbeitenden}

\section{A11.1 Unterschiede in der Gruppe}

Es werden Karten verteilt, auf die jeder Teilnehmende (TN) eine Sache über sich schreibt, von der er der Überzeugung ist, dass sie ihn von den anderen unterscheidet. Die Seminarleitung sammelt die Karten ein und vermischt sie. Anschließend werden sie wieder an die TN verteilt. Nacheinander werden die Karten vorgelesen und die Besitzer_innen ermittelt. Hier ist wichtig, dass nicht gleich mit der Wahrheit herausgerückt wird, sondern die Gruppe die Entscheidung mehrfach verändern kann. 


\section{A11.2 Das Haus in der Müllerstraße ${ }^{9}$}

Die Seminarteilnehmenden bekommen den Arbeitsauftrag, dass in der »Müllerstraße « neue Häuser gebaut werden. Die Teilnehmenden sollen darauf achten, dass bei den neuen Bewohner_innen möglichst viele unterschiedliche Lebenssituationen von Kindern berücksichtigt werden. Dafür können auch Vorgaben gemacht werden (in einer Wohnung lebt eine Wohngemeinschaft, in einer weiteren leben zwei Frauen mit einem Kind ...). Wenn ein ganzer Straßenzug gestaltet wird, könnten neben Mietshäusern auch Eigenheime vorkommen. Die TN sollten möglichst genau die Lebensumstände der Familien und Einzelpersonen beschreiben und künstlerisch darstellen.

Im anschließenden Gespräch können vertiefende Fragen zu den Lebensumständen der Personen an die Teilnehmenden gestellt werden. Dabei können Gemeinsamkeiten und Unterschiede herausgearbeitet werden.

Die »Müllerstraße « kann im weiteren Verlauf der Seminarwoche weiter bearbeitet werden und es kann immer wieder Bezug darauf genommen werden.

\section{A11.3 Reduktion ${ }^{10}$}

Zu einem beliebigen Thema (Sexualität, Aids, Partnerschaft, Name der Einrichtung ...) wird eine Assoziationskette gebildet.

1. Gruppe ruft 16 Begriffe zum Thema, die auf ein Flipchart geschrieben werden. Jeder zuerst gehörte Begriff gilt. Es wird nicht gewertet.

2. Aus 1 und 2 wird wieder ein Begriff assoziiert, folgend aus 3 und 4, 5 und 6 usw. Es bleiben 8 Begriffe.

3. Aus diesen 8 Begriffen werden wieder zwei zu einem. Es bleiben 4 Begriffe.

9 Quelle: in Anlehnung an Jutta Hartmann (2008). In S. Timmermanns \& E. Tuider (2008). Sexualpädagogik der Vielfalt. Praxismethoden zu Identitäten, Beziehungen, Körperund Prävention für Schule und Jugendarbeit. Weinheim: Beltz Juventa, S. $47 f$.

10 Quelle: Seminar »Sexualität und Behinderung « an der HS Merseburg, Dozentin: Petra Winkler (vgl. Gilbers/Winkler, 2006). 
4. Aus diesen 4 Begriffen werden 2.

5. Aus 2 wird ein Begriff.

\section{A11.4 Zielscheibe ${ }^{11}$}

Die Methode dient dazu, über die gewählten Begrifflichkeiten zu diskutieren. Im Rahmen des Seminars kann sie als Einstieg dienen, über die Erwartungen von Teilnehmenden an eine Freizeit ins Gespräch zu kommen.

Dazu werden mehrere Zielscheiben mit je einem Begriff überschrieben (Beispiel: Freie Zeit, gutes Programm, Regeln, Freundschaften ...).

Jede_r Seminarteilnehmer_in erhält einen Stift oder eine entsprechende Anzahl von Klebepunkten.

Die Klebepunkte werden je nach eigenem Empfinden der »Wichtigkeit $\ll$ an die Stelle der Zielscheibe geklebt (Zentrum = am wichtigsten, äußerer Ring = nicht so wichtig/unwichtig).

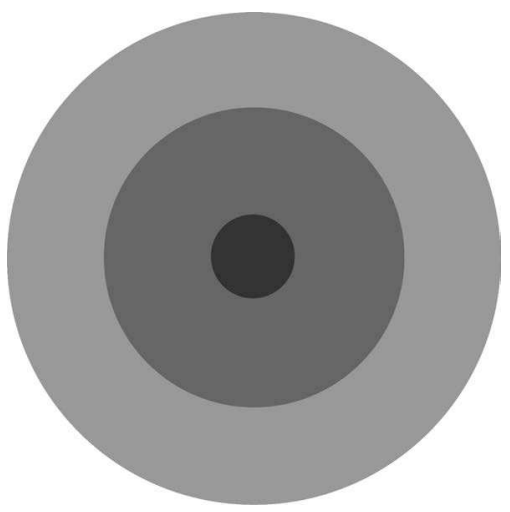

Abb. 2: Zielscheibe

11 Quelle: Seminar »Altersspezifische Sexualpädagogik - Methoden für die Zielgruppe 10-14 Jahre« an der HS Merseburg, Dozent_in: Kathrin Gebhardt, Ken Kupzok. 


\section{A11.5 »Stop« - Übung zu Nähe und Distanz ${ }^{12}$}

Die Seminarteilnehmenden werden in zwei Gruppen geteilt und stellen sich möglichst weit auseinander. Zwischen den einzelnen Personen ist ausreichend Abstand. Es bilden sich gegenüberstehende Pärchen. Nach jeder Aktion bleibt die jeweilig agierende Gruppe kurz stehen, schaut sich um und geht anschließend wieder auf die Ausgangsposition zurück. Es darf im Folgenden nicht gesprochen werden.

$>$ A geht auf B zu. A bleibt irgendwann stehen, oder B sagt deutlich STOP.

$>\quad$ B geht auf A zu. B bleibt irgendwann stehen, oder A sagt deutlich STOP.

$>$ A geht auf B zu. B hat die Augen geschlossen. A bleibt irgendwann stehen, oder B sagt deutlich STOP.

$>$ B geht auf A zu. A hat die Augen geschlossen. B geht auf A zu. B bleibt irgendwann stehen, oder A sagt deutlich STOP.

$>$ A geht auf B zu. A hat dabei die Augen geschlossen. A bleibt irgendwann stehen, oder $B$ sagt deutlich STOP.

$>$ B geht auf A zu. B hat dabei die Augen geschlossen. B geht auf A zu. B bleibt irgendwann stehen, oder A sagt deutlich STOP.

$>$ A geht auf B zu. B befindet sich in der Hocke. A bleibt irgendwann stehen, oder B sagt deutlich STOP.

$>$ B geht auf A zu. A befindet sich in der Hocke. B geht auf A zu. B bleibt irgendwann stehen, oder A sagt deutlich STOP.

Ziel: Jede_r kann Gefühle wahrnehmen. Es gibt ein Gefühl für Körpergrenzen, Autonomie, Integrität, Selbstbewusstsein (» was will ich, was will ich nicht «). Das kann aberzogen werden (durch Zwang, Strafe, aber auch Belohnung). Die Übung soll das Gefühl für etwas wollen/etwas nicht wollen, für Nähe und Distanz, für die Wahrnehmung eigener Grenzen und die Grenzen anderer aktivieren.

Im anschließenden Gespräch kann/muss auf das individuelle Grenzempfinden eingegangen und die Empfindungen der Teilnehmenden in den einzelnen Situationen zur Sprache gebracht werden. Wichtig emp-

12 Quelle: Seminar »Sexuelle Gewalt« an der HS Merseburg, Dozent_in: Elke Lieback, Prof. Dr. Konrad Weller. 
finde ich bei der Übung, dass nicht gesprochen wird und der Austausch darüber wirklich erst in der Auswertungsrunde stattfindet.

\section{A11.6 Baummethode ${ }^{13}$}

Für die Baummethode benötigt man einen im Vorfeld gemalten Baum. Gut eignen sich dafür Wachsmalstifte oder, wenn die Vorbereitungszeit ausreichend ist, auch Fingerfarbe und eine Rolle Packpapier als Grundlage. So kann der Baum auch in einer entsprechenden Größe gemalt werden.

Die Methode eignet sich als Rahmen für eine Tagesveranstaltung. Im ersten Teil werden die Erwartungen und Fragen der Teilnehmenden abgerufen, im zweiten Teil erhält die Leitung ein Feedback.

\section{Teil 1}

Jeder macht sich Notizen zu folgenden Fragestellung:

$>$ Was möchte ich ernten? (Welche Fragen habe ich?) (orange)

$>$ Auf welchem Grund stehe ich? (Welche Vorerfahrungen habe ich?) (grün)

Das Vorstellen der eigenen Kärtchen kann mit der Namensvorstellung verbunden werden. Dabei werden die orangenfarbenen Kärtchen in die Baumkrone gehängt (Früchte), die grünen Kärtchen im Wurzelbereich.

\section{Teil 2}

Die Teilnehmenden schreiben auf Kärtchen zu folgenden Fragestellungen ihr Feedback:

> Welche Früchte konnte ich ernten? (Erkenntnisgewinn) (rot)

$>$ Welche Knospen sind nicht aufgegangen? (Was hat mir gefehlt?) (gelb)

$>$ Was möchte ich hier lassen? (weiß)

13 Quelle: Nach einer Methode aus dem Seminar »Sexualpädagogische Methoden für Jugendliche ab 14 Jahren« an der HS Merseburg, Dozent_in: Barbara Trapp, Uwe Tüffers. 
Auch hier erfolgt die Auswertung im Plenum. Dabei können die Erkenntnisgewinne (rote Karten) in den Korb gelegt, die »Knospen « an den Baum gehängt und die weißen Kärtchen im Wurzelbereich abgelegt werden.

\section{A11.7 Begriffe zur sexuellen Entwicklung (Zeitstrahl) - Meilensteine der sexuellen Entwicklung}

Die vorgeschlagenen Begriffe können auch mit den Entwicklungsphasen von Sigmund Freud und den Entwicklungsaufgaben nach Erik H. Erikson und Robert H. Havighurst kombiniert werden.

\begin{tabular}{|c|c|}
\hline Entwicklung der Geschlechtsteile & $\begin{array}{l}\text { die Eizellen beim Mädchen } \\
\text { entstehen }\end{array}$ \\
\hline $\begin{array}{l}\text { Beginn der sinnlichen und } \\
\text { lustvollen Erfahrungen }\end{array}$ & erste Erektionen des Penis \\
\hline Feuchtwerden der Scheide & $\begin{array}{c}\text { Sexualität ist von } \\
\text { Sinneserfahrungen geprägt }\end{array}$ \\
\hline $\begin{array}{l}\text { Hautkontakt sehr wichtig für die } \\
\text { Entwicklung }\end{array}$ & Mund als Lustorgan \\
\hline Entdeckung des eigenen Körpers & $\begin{array}{c}\text { Lustempfinden durch Berührung } \\
\text { der Geschlechtsorgane }\end{array}$ \\
\hline $\begin{array}{l}\text { Interesse am Thema } \\
\text { Schwangerschaft }\end{array}$ & sich nackt zeigen \\
\hline $\begin{array}{l}\text { Genitalien werden zu } \\
\text { erogenen Zonen }\end{array}$ & $\begin{array}{l}\text { Interesse am anderen Geschlecht, } \\
\text { Neugierde }\end{array}$ \\
\hline $\begin{array}{l}\text { Rivalitätsgefühl mit gleichge- } \\
\text { schlechtlichem Elternteil }\end{array}$ & $\begin{array}{c}\text { es entwickelt sich } \\
\text { ein ausgeprägtes Schamgefühl }\end{array}$ \\
\hline $\begin{array}{l}\text { Entstehung von Moral und } \\
\text { schlechtem Gewissen }\end{array}$ & $\begin{array}{l}\text { Witze über Sexualität (Vulgär- und } \\
\text { Fäkalsprache) werden gemacht }\end{array}$ \\
\hline $\begin{array}{c}\text { scheinbare Unterbrechung der bis- } \\
\text { herigen sexuellen Entwicklung }\end{array}$ & $\begin{array}{l}\text { Ausgestaltung der eigenen } \\
\text { Geschlechterrolle }\end{array}$ \\
\hline $\begin{array}{l}\text { überwiegend gleichgeschlechtliche } \\
\text { Freundschaften }\end{array}$ & $\begin{array}{c}\text { sprachliche } » \text { sexualisierte } \ll \\
\text { Ausdrucksweise }\end{array}$ \\
\hline $\begin{array}{l}\text { Interesse am anderen Geschlecht } \\
\text { wächst wieder }\end{array}$ & $\begin{array}{l}\text { erstes Verliebtsein mit großen } \\
\text { Gefühlen }\end{array}$ \\
\hline
\end{tabular}




\begin{tabular}{|c|c|}
\hline $\begin{array}{l}\text { Beginn der körperlichen } \\
\text { Veränderungen beim Mädchen }\end{array}$ & Becken bei Mädchen wird breiter \\
\hline Brust beginnt sich zu entwickeln & $\begin{array}{l}\text { Schambehaarung bei } \\
\text { Mädchen wächst }\end{array}$ \\
\hline $\begin{array}{l}\text { Wachstumsbeginn von Scheide und } \\
\text { Gebärmutter }\end{array}$ & $\begin{array}{l}\text { großer Wachstumsschub } \\
\text { bei Mädchen }\end{array}$ \\
\hline volle Brustentwicklung & $\begin{array}{c}\text { Beginn der körperlichen Verände- } \\
\text { rungen bei Jungen }\end{array}$ \\
\hline Peniswachstum & erste Schambehaarung beim Jungen \\
\hline $\begin{array}{l}\text { erster pubertärer Längenwachs- } \\
\text { tumsschub beim Jungen }\end{array}$ & $\begin{array}{l}\text { Ende des Längenwachstums } \\
\text { beim Jungen }\end{array}$ \\
\hline Stimmbruch bei Jungen & erste Menstruation (Menarche) \\
\hline erste Samenzellen (Spermarche) & Ablösung vom Elternhaus \\
\hline körperliche Vergleiche mit anderen & $\begin{array}{c}\text { Unsicherheit im Umgang } \\
\text { mit anderen }\end{array}$ \\
\hline Barthaare wachsen & $\begin{array}{l}\text { markanter Stimmwechsel } \\
\text { bei Jungen }\end{array}$ \\
\hline $\begin{array}{l}\text { Entwicklung eigener } \\
\text { Wertevorstellungen }\end{array}$ & das erste Mal \\
\hline $\begin{array}{l}\text { sich ausprobieren im Bezug auf } \\
\text { Sexualität }\end{array}$ & Familienplanung \\
\hline Midlife-Crises & $\begin{array}{l}\text { Veränderung der Sexualität } \\
\text { (kann mehrfach vorkommen) }\end{array}$ \\
\hline Wechseljahre & Rentenbeginn \\
\hline körperlicher Abbau & geistiger Abbau \\
\hline Ende der Sexualität bei der Frau & Ende der Sexualität beim Mann \\
\hline
\end{tabular}




\section{A11.8 Arbeitsblatt: »|st das Kindeswohlgefährdung?«14}

\section{Gibt es hier gewichtige Anzeichen von Kindeswohlgefährdung?}

$\uparrow \mathrm{Ja}-\downarrow$ Nein $-\rightarrow$ Vielleicht

1 Bei dem Highlight des zweiwöchigen Waldheims (Ferienangebot ohne Übernachtung), der gemeinsamen Übernachtung auf dem Gelände am vorletzten Tag, fehlt ein Mädchen, bei dem euch schon die ganze Zeit die immer gleiche schlampige Kleidung, schlechte Zähne und ein deutlicher Körpergeruch aufgefallen sind.

2 Ein 14-jähriges Mädchen zeigt in der Mittagspause den anderen Mädchen in ihrem Zimmer Nackt-Fotos von sich herum, die ihr Onkel am FKK-Strand von ihr gemacht hat.

3 Nach zwei Wochen im Ferienangebot fällt den Betreuer_innen auf, dass ein stiller zehnjähriger Junge ein paarmal anderen Kindern gegenüber aggressiv geworden ist. Beim Ausflug in den Wald sammeln die Kinder kleine Tiere und Pflanzen in Gläsern. Die Betreuer_innen finden den Jungen später am Tag, wie er die Tiere grausam quält. Aufgefallen ist auch, dass er viele blaue Flecken an den Armen und Beinen hat.

4 Auf der Freizeit fällt auf, dass drei Geschwister einer Familie im Alter von 8-10 nicht in der Lage sind, mit Besteck zu essen, und sich trotz entsprechender Ermahnungen permanent mehr Essen aufladen, als sie bewältigen können.

5 Der 15-jährige Erwin gebraucht seit Beginn des Lagers in deiner Gegenwart in jedem zweiten Satz Begriffe wie vögeln, ficken, fotzen stopfen, pimpern ...

6 Ein 13-jähriges Mädchen erzählt auf der Freizeit, dass sie zu Hause manchmal den Haushalt erledigen und die drei jüngeren Geschwister versorgen muss. Besonders schlimm wird es ihrer Erzählung nach, wenn die Mama "wieder mal krank« ist und in die Klinik muss. Der Papa, der viel arbeitet und oft weg ist, habe ihr erklärt, dass es in diesen Situationen an ihr liegt, ob es der Familie gut geht oder nicht und ihr angedroht, dass sie nicht mehr mit ihren Freundinnen spielen darf, wenn sie es nicht schafft.

7 Ein 13-jähriges Mädchen erzählt, dass bei ihnen Zuhause immer im Ehebett gekuschelt wird und der Vater ihr dabei auch schon mal unter dem Nachthemd den Bauch gestreichelt hat.

8 Bei der Abfahrt zur Freizeit küsst eine Mutter ihren 16-jährigen Sohn auf den Mund.

9 Der 15-jährige Sohn erzählt, dass er zu Hause gemeinsam mit den Eltern einen Pornofilm auf DVD angeschaut hat.

10 Die Begründung des neunjährigen Jungen, woher seine Hämatome rühren, die ihr beim Baden an ihm wahrgenommen habt, stimmt nicht mit der Erklärung der Mutter überein, die ihr am Abend befragt.

14 Quelle: Schulungsmaterial der Evangelischen Jugend Stuttgart. 
Diese Fragestellungen lassen sich auch sehr gut als Soziometrieübungen gestalten. Dafür wird eine Situation vorgelesen und die Seminarteilnehmenden müssen sich auf einer gedachten Linie positionieren, wobei das eine Ende die absolute Kindeswohlgefährdung markiert und das andere Ende die entgegengesetzte Meinung. Es kann ein Austausch über die eigene Positionierung erfolgen. 
Torsten Linke

Sexualität und Familie Möglichkeiten sexueller Bildung im Rahmen erzieherischer Hilfen

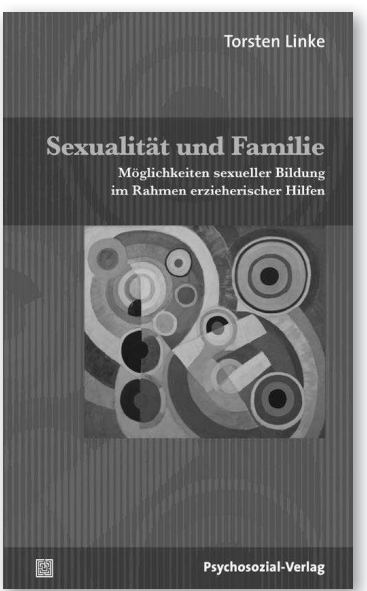

$2015 \cdot 109$ Seiten $\cdot$ Broschur ISBN 978-3-8379-2468-8

\begin{abstract}
Obwohl sexuelle Themen ein wichtiger Bestandteil der sozialpädagogischen Familienberatung und der Kinder- und Jugendhilfe sind, gibt es nur wenige Veröffentlichungen zum Thema.
\end{abstract}

Auch in der konkreten Praxis der Sozialen Arbeit sind theoretische Konzepte für sexuelle Bildung und Beratung ungenügend verankert. Dieser Lücke wendet sich der vorliegende Band zu: Ausgehend von der Studie »Partner 4« zu Jugendsexualität liefert er nicht nur allgemeine Anregungen für die Beratungspraxis, sondern unterbreitet auch Vorschläge für Konzepte, die den diversen und komplexen Lebenslagen der Kinder und Jugendlichen Rechnung tragen. Der Fokus liegt dabei auf der Sozialisationsinstanz Familie. 


\section{Manuela Tillmanns \\ Intergeschlechtlichkeit Impulse für die Beratung}

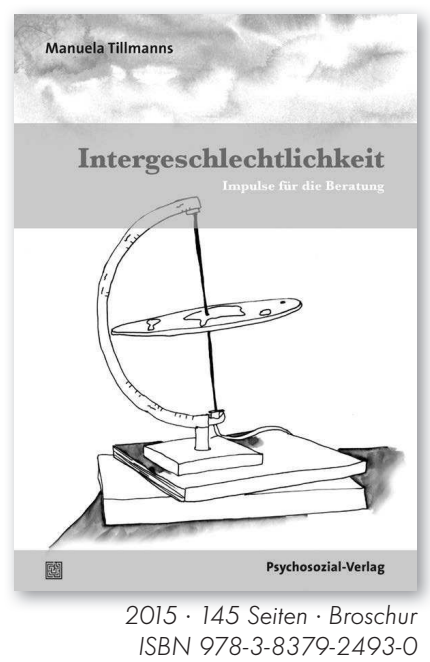

Menschen, die nicht eindeutig dem männlichen oder weiblichen Geschlecht zugeordnet werden können, sind gesellschaftlichen Stigmatisierungen und medizinisch-psychologischen Pathologisierungen ausgesetzt.
Zentrale Studien im deutschsprachigen Raum bestätigen die Notwendigkeit eines fundamentalen Paradigmenwechsels im gesellschaftlichen Umgang mit Intergeschlechtlichkeit bzw. Intersexualität. Zwar hat die Auseinandersetzung mit dem Thema bereits Einzug in den Bildungsbereich erhalten, jedoch existieren bislang keine Veröffentlichungen zur professionellen Beratung von Inters*.

Mithilfe der Analyse von Selbstdarstellungen und Expert_innen-Interviews werden im vorliegenden Buch konkrete Impulse und Handlungsempfehlungen für eine inter*-spezifische Beratungspraxis erarbeitet. Dabei werden auch bisher bestehende Beratungsansätze und -konzepte aufgegriffen und unter Einbezug von Peers und Selbsthilfegruppen erweitert. So ergeben sich neue methodische Zugänge und Perspektiven, die sich an den Bedürfnissen und Wünschen intergeschlechtlicher Personen orientieren und ihnen Formen der Selbstermächtigung sowie barrierefreie Räume für selbstbestimmtes Agieren eröffnen.

\section{Walltorstr. 10 - 35390 Gießen · Tel. 0641-969978-18 - Fax 0641-969978-19} bestellung@psychosozial-verlag.de 


\section{Psychosozial-Verlag}

Julia Sparmann

\section{Körperorientierte Ansätze für die Sexuelle Bildung junger Frauen Eine interdisziplinäre Einführung}

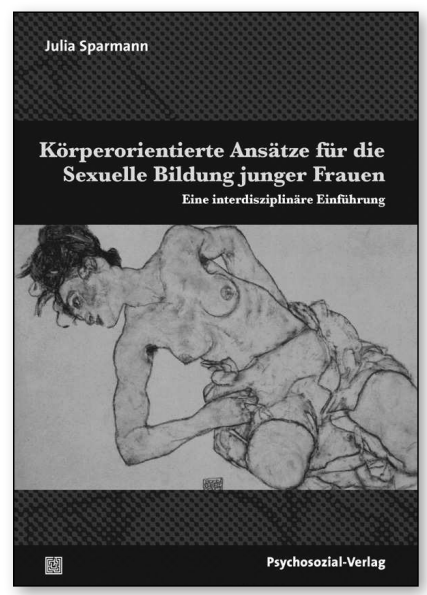

Dezember $2015 \cdot 113$ Seiten · Broschur ISBN 978-3-8379-2519-7

Fundierte Grundlagenarbeit, fachliche Auseinandersetzung, interdisziplinäre Offenheit - ein neuer Zugang zur Sexuellen Bildung junger Frauen.
Junge Frauen stehen mit ihrem Körpergefühl und sexuellem Erleben im Spannungsfeld individuell biografischer Erfahrungen und gesellschaftlicher Ideale. Sie besitzen in der heutigen Zeit ein ausgeprägtes Körperbewusstsein, doch statt körperlichen Wohlbefindens stehen oft kritische Selbstbewertung und Kontrolle im Vordergrund. Körperorientierte Methoden stärken über sinnlich-konkrete Selbsterfahrung die Körperwahrnehmung und erweitern sexuelle Fähigkeiten und Potenziale.

Julia Sparmann verbindet erprobte Methoden aus Körperpsychotherapien, dem sexualtherapeutischen Ansatz Sexocorporel und Tantra zu einem Konzept körperorientierter Herangehensweisen für die Sexuelle Bildung. Ressourcenorientiert werden die Hintergründe der Ansätze reflektiert und im Sinne einer emanzipatorischen Sexuellen Bildung modifiziert. Es wird deutlich, dass die Vermittlung der komplexen Zusammenhänge zwischen Atem, Muskeltonus und Bewegung sowie die Betonung eines achtsamen Körperzugangs dabei eine große Bereicherung darstellen. 
Im vorliegenden Buch stehen der Kinderschutz und die Prävention von sexualisierter Gewalt in der verbandlichen Jugendarbeit im Fokus. Miriam Günderoth vermittelt Basiswissen für all diejenigen, die in der Jugendarbeit tätig sind oder mit diesem Tätigkeitsfeld im regen Austausch stehen.

Die Autorin geht auf die Spezifika der verbandlichen Jugendarbeit ein und erklärt die gesetzlichen Bestim- mungen für diesen Arbeitsbereich. Sie gibt Anregungen für die Auseinandersetzung mit und für die Entwicklung von verbandsspezifischen Regelungen und Schulungskonzepten. Zudem macht sie deutlich, wie ein angemessener Umgang mit Kindern und Jugendlichen gestaltet werden kann und wie Mitarbeiter und Ehrenamtliche für das Thema Prävention von sexualisierter Gewalt sensibilisiert werden können.

Miriam Günderoth ist Diplom-Sozialpädagogin und DiplomReligionspädagogin und studierte berufsbegleitend "Sexualpädagogik und Familienplanung». Sie ist Mitarbeiterin in der Koordinierungsstelle »Prävention sexualisierter Gewalt « der Evangelischen Landeskirche in Württemberg. Freiberuflich ist sie in der Bildungsarbeit tätig und leitet Schulungen für JugendleiterInnen und -referentInnen zum Thema Kinderschutz und sexualisierte Gewalt sowie zur Sexualpädagogik im Rahmen von Lehraufträgen. 